\title{
Symmetry-protected entangling boundary zero modes in crystalline topological insulators
}

\author{
Po-Yao Chang ${ }^{1}$, Christopher Mudry ${ }^{2}$, Shinsei Ryu ${ }^{1}$ \\ ${ }^{1}$ Department of Physics, University of Illinois at Urbana-Champaign, Urbana, IL 61801, USA \\ ${ }^{2}$ Condensed Matter Theory Group, Paul Scherrer Institute, CH-5232 Villigen PSI, \\ Switzerland \\ E-mail: chang153eillinois.edu
}

\begin{abstract}
Crystalline topological insulators owe their topological character to the protection that certain boundary states acquire because of certain point-group symmetries. We first show that a Hermitian operator obeying supersymmetric quantum mechanisms (SUSY QM) delivers the entanglement spectrum. We then show that such an entanglement spectrum that is compatible with a certain point-group symmetry obeys a certain local spectral symmetry. The latter result is applied to the stability analysis of four fermionic non-interacting Hamiltonians, the last of which describes graphene with a Kekule distortion. All examples have the remarkable property that their entanglement spectra inherit a local spectral symmetry from either an inversion or reflection symmetry that guarantees the stability of gapless boundary entangling states, even though all examples fail to support protected gapless boundary states at their physical boundaries.
\end{abstract}




\section{Contents}

1 Introduction

2 Symmetries and entanglement spectrum 6

2.1 Non-interacting fermions . . . . . . . . . . . . . . . . . . 6

2.1.1 Hamiltonian ................. 6

2.1.2 Entanglement spectrum of the equal-time one-point correlation matrix 8

2.1.3 Equal-time one-point correlation matrix and SUSY QM . . . . . . 10

2.1.4 Chiral symmetry of the entanglement spectrum $\sigma\left(Q_{A}\right) \ldots \ldots$

2.1.5 Equal-time one-point correlation matrix and $\mathscr{P} \mathscr{C} \mathscr{T}$ symmetry . . . 17

2.2 Interacting fermions . . . . . . . . . . . . . . . 20

3 Physical versus entangling boundaries, spectral gap, and locality 24

3.1 Spectral gap and locality of the equal-time one-point correlation matrix . . . 25

3.2 Spectral gap and locality of the spectral symmetry $(2.45 \mathrm{~d}) \ldots \ldots$

4 Topological insulator protected by reflection (inversion) symmetry in one dimension 27

4.1 Hamiltonian . . . . . . . . . . . . . . . . . . 27

4.2 Symmetries . . . . . . . . . . . . . . . . . . . 29

4.3 Partition, topological numbers, and zero modes . . . . . . . . . . 33

4.4 Stability analysis of the zero modes . . . . . . . . . . . . . . . 37

4.5 Numerical verification that $\Gamma_{\mathscr{P} A}$ is local . . . . . . . . . . . . 41

5 Topological insulator protected by one reflection symmetry in two dimensions 42

5.1 Hamiltonian and topological quantum numbers . . . . . . . . . . . . 42

5.2 Symmetries . . . . . . . . . . . . . . . . . . . 43

5.3 Partition and zero modes . . . . . . . . . . . . . . . . 44

5.4 Stability analysis of the zero modes . . . . . . . . . . . . . . 46

5.4.1 Definitions of $\widetilde{\mathcal{H}}_{i \mu \nu}$ and $\widetilde{\mathcal{Q}}_{k_{i} \mu \nu A_{i+1}} \ldots \ldots \ldots \ldots$

$5.4 .2 \quad \operatorname{Spectra} \sigma\left(\tilde{\mathcal{H}}_{i \mu \nu}\right) \ldots \ldots \ldots \ldots \ldots$

$5.4 .3 \quad \operatorname{Spectra} \sigma\left(\widetilde{\mathcal{Q}}_{\mu \nu A_{i+1}}\right) \ldots \ldots \ldots \ldots \ldots \ldots$

5.4.4 Spectra $\sigma\left(\mathcal{H}_{k_{i} \mathscr{R} ; \mathscr{S}}\right)$ and $\sigma\left(Q_{k_{i} \mathscr{R} ; \mathscr{S} A_{i+1}}\right) \ldots \ldots \ldots \ldots$

5.5 Existence of spectral flows in the entanglement spectra . . . . . . . . 55

6 Topological band insulator protected by two reflection symmetries in two dimensions

6.1 Hamiltonian and topological quantum numbers . . . . . . . . . . . . 58

6.2 Symmetries ........................ 59

6.3 Partition and zero modes . . . . . . . . . . . . . . 61

6.4 Stability analysis of the zero modes . . . . . . . . . . . . . . . . . 64

6.4.1 Hamiltonian spectrum ................ 64 
6.4.2 Entanglement spectrum ................... 65

7 Graphene with Kekule order as an inversion-symmetric topological insulator in two dimensions 66

7.1 Introduction . . . . . . . . . . . . . . . . . . . 66

7.2 Hamiltonian .......................... 67

7.3 Symmetries . . . . . . . . . . . . . . . . . 69

7.4 Partition .......................... 69

7.5 Kekule with armchair edges . . . . . . . . . . . . 71

7.5.1 Time-reversal symmetry breaking ............ 72

7.5.2 Sublattice symmetry breaking ............... 73

7.5.3 Inversion symmetry breaking . . . . . . . . . . . 73

7.5.4 Stability analysis of the zero modes . . . . . . . . . . . . 74

7.6 Rotated Kekule with armchair edges . . . . . . . . . . . . . . . . . . 76

7.6.1 Rotated Kekule distortion . . . . . . . . . . . . . 77

7.6.2 Spectra for rotated Kekule . . . . . . . . . . . . . . . . . 79

7.7 Kekule with zigzag edges . . . . . . . . . . . . . . . . . 79

7.7 .1 Hamiltonian . . . . . . . . . . . . . . . 79

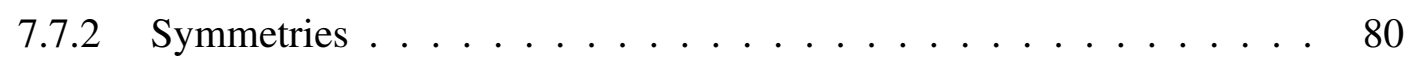

7.7 .3 Partition . . . . . . . . . . . . . . . 82

7.7.4 Hamiltonian and entanglement spectra . . . . . . . . . . . . 82

7.7.5 Stability analysis of the zero modes ............ 83

7.8 Counting the mid-gap states protected by inversion symmetry . . . . . . . 85

8 Conclusion 87 


\section{Introduction}

One of the fundamental distinctive feature of phases of matter is the spontaneous breaking of symmetries. As a corollary, phases of matter are gapless under very general conditions when the broken symmetry group is continuous. There are, however, incompressible phases of matter at zero temperature that are featureless from the point of view of spontaneous symmetry breaking. Examples thereof are the integer quantum Hall effect (IQHE) and topological insulators (superconductors). [1, 2] Such incompressible phases of matter are inherently quantum mechanical. They have no classical counterparts, unlike phases breaking spontaneously a symmetry. They are characterized by topological attributes such as a quantized response function or the existence of (symmetry protected) gapless modes that propagate along a physical boundary, while they are exponentially localized away from the physical boundary (in short gapless boundary modes or edge states), when the ground state is non-degenerate in the thermodynamic limit. Another probe of their topological character is the entanglement of their incompressible ground state, [3, 4, 5, 6, 7, 8, 9, 10, 11] whether short ranged when the ground state is non-degenerate (as in the IQHE), or long ranged when the ground states becomes degenerate in the thermodynamic limit [as in the fractional quantum Hall effect (FQHE)].

One probe that measures the entanglement of an incompressible ground state $|\Psi\rangle$ is the entanglement entropy (von Neumann entropy) defined by

$$
S_{A}:=-\operatorname{tr}_{A}\left(\hat{\rho}_{A} \ln \hat{\rho}_{A}\right) .
$$

Here, the total system is divided into two subsystems $A$ and $B$,

$$
\hat{\rho}_{A}:=\operatorname{tr}_{B}|\Psi\rangle\langle\Psi|
$$

is the reduced density matrix obtained by tracing over the states in subsystem $B$ of the total density matrix

$$
\hat{\rho}:=|\Psi\rangle\langle\Psi|
$$

in the incompressible ground state $|\Psi\rangle$. In this paper, we will almost exclusively consider single-particle Hamiltonians, their non-degenerate incompressible ground states $|\Psi\rangle$, and partitionings into $A$ and $B$ with respect to a referred basis for which locality is manifest. We will also assume that it is possible to associate with $A$ and $B$ two regions of $d$-dimensional position space sharing a $(d-1)$-dimensional boundary. This boundary is called the entangling boundary of the partition into $A$ and $B$. This entangling boundary is unrelated to any physical boundary selected by the choice of open boundary conditions.

Another probe for the entanglement contained in an incompressible ground state $|\Psi\rangle$ is the entanglement spectrum. [3, 6, 7, 8] On the one hand, topological phases that are characterized by the presence of gapless boundary states in the energy spectrum must support gapless modes that propagate along the entangling boundary but decay exponentially fast 
away from the entangling boundary (in short entangling boundary states or entangling edge modes) in the entanglement spectrum. [3, 12, 8, 13] On the other hand, there are symmetryprotected topological phases that do not show gapless boundary modes in the energy spectrum, while they do show gapless boundary modes in the entanglement spectrum. The entanglement spectrum can thus be thought of as a more refined diagnostic for identifying and classifying topological incompressible ground states than the energy spectrum. For example, noninteracting insulators that owe their topological character to the existence of an inversion symmetry, may support mid-gap states in the entanglement spectrum that are protected by the inversion symmetry, while they need not support gapless boundary modes in the energy spectrum. These mid-gap modes in the entanglement spectrum cannot be removed by an adiabatic and local deformation of the single-particle Hamiltonian. As such there existence may be used as a mean to quantify a topological invariant. Symmetry protected topological phases can also arise from other discrete symmetries, such as reflection symmetry, [14, 15, 16, 17, 18, 19, 20, 21] or more general point-group symmetries. [22, 23, 24, 25, 26] Moreover, it is known that the eigenvalues of discrete symmetry operators at the symmetric points in the Brillouin zone (BZ) are related to the number of the mid-gap states in the entanglement spectrum for inversion- and point-group-symmetric topological ground states. [13, 23, 24, 25].

In this paper, we explain how crystalline symmetries of single-particle Hamiltonians with an incompressible ground state manifest themselves in the entanglement spectrum and how they can protect gapless boundary states in the entanglement spectrum, when no such protection is operative for the boundary states in the energy spectrum due to the non-local character of crystalline symmetries.

This paper is organized as follows. In Sec. 2, after some preliminary definitions in Secs. 2.1.1 and 2.1.2, we show that the entanglement Hamiltonian is an example of supersymmetric quantum mechanics in Sec. 2.1.3. The main result of this paper follows in Sec. 2.1.4, where we show under what conditions a crystalline symmetry can induce a spectral symmetry of the entanglement spectrum that is absent from the energy spectrum of a single-particle Hamiltonian with an incompressible ground state. [12, 13, 27] The subtle interplay between the geometry imposed by the boundary conditions, the non-local crystalline symmetry, and its (local) realization on the physical (entangling) boundaries is explained in Sec. 3. We then apply our main result, Eqs. (2.45) and (2.52), to the stability analysis of gapless edge states in the energy and entanglement spectra for four examples of single-particle Hamiltonians with incompressible ground states in Secs,4, 5, 6, and 7, respectively. This stability analysis is the most intricate when treating a one-dimensional tight-binding model with inversion symmetry in Sec. 4. Two copies of a pair of Chern insulators differing by the sign of their Chern numbers that accommodate a reflection symmetry are treated in Sec. 5 as the first two-dimensional example. By considering two copies of the filled lowest Landau level with opposite Chern numbers, we treat the example of two reflection symmetries in two dimensions in Sec. 6. We close the applications of Eqs. 2.45) and (2.52) by demonstrating that the Kekule distortion of graphene (see Ref. [28]) should be thought of as a crystalline topological insulator. We conclude in Sec. 8 . 


\section{Symmetries and entanglement spectrum}

The goal of this section is to study the relationship between the symmetries shared by a fermionic Hamiltonian and its ground state and the symmetries of the reduced density matrix of the pure-state density matrix constructed from the ground state, whereby the reduced density matrix presumes a partitioning of the fermionic Fock space into the tensor product of two Fock subspaces, i.e., the decomposition of the single-particle Hilbert space into the direct sum of two subspaces.

We shall begin by studying the non-interacting case in Sec. 2.1. The non-interacting limit is defined in Sec. 2.1.1. The ground state for $N_{\mathrm{f}}$ fermions hopping between the sites and orbitals of a lattice is a Slater determinant (Fermi sea). By Wick's theorem, all the information contained in the density matrix for the Fermi sea can be retrieved from the equaltime one-point correlation function (matrix) and conversely, as is reviewed in Sec. 2.1.2. The direct sum decomposition of the single-particle Hilbert space is an example of a graded vector space. We show in Sec. 2.1.3 that this grading allows to construct from the equal-time onepoint correlation matrix a supersymmetric single-particle Hamiltonian. This supersymmetry has consequences for the spectral properties of the reduced density matrix (entanglement spectrum) as is shown in Sec. 2.1.4, where we demonstrate that certain symmetries of the Hamiltonian induce symmetries of the entanglement spectrum. Conversely, we show in Sec.2.1.5 that a spectral symmetry of the Hamiltonian can turn into a symmetry of the reduced density matrix in view of the hidden supersymmetry of the latter. Spectral symmetry of the Hamiltonian can thus turn into degeneracies of the entanglement spectrum.

The interacting case is considered in Sec. 2.2. We show how a symmetry of the Hamiltonian and of its ground state that interchanges the partition is realized on the reduced density matrix.

\subsection{Non-interacting fermions}

2.1.1. Hamiltonian We consider a lattice $\Lambda \subset \mathbb{Z}^{d}$ whose $N$ sites are labeled by the vector $r:=\left(r_{1}, \cdots, r_{N}\right)^{\top}$. Each site $r$ is also associated with $N_{\text {orb }}$ orbital (flavors) labeled by the Greek letter $\alpha=1, \cdots, N_{\text {orb }}$. We define the non-interacting second-quantized Hamiltonian

$$
\hat{H}:=\sum_{\boldsymbol{r}, \boldsymbol{r}^{\prime}=1}^{N} \sum_{\alpha, \alpha^{\prime}=1}^{N_{\text {orb }}} \hat{\psi}_{\alpha, \boldsymbol{r}}^{\dagger} \mathcal{H}_{\alpha, \boldsymbol{r} ; \alpha^{\prime}, \boldsymbol{r}^{\prime}} \hat{\psi}_{\alpha^{\prime}, \boldsymbol{r}^{\prime}} .
$$

The pair of creation $\left(\hat{\psi}_{\alpha, r}^{\dagger}\right)$ and annihilation operators $\left(\hat{\psi}_{\alpha^{\prime}, r^{\prime}}\right)$ obey the fermion algebra

$$
\begin{aligned}
& \left\{\hat{\psi}_{\alpha, \boldsymbol{r}}^{\dagger}, \hat{\psi}_{\alpha^{\prime}, \boldsymbol{r}^{\prime}}\right\}=\delta_{\alpha, \alpha^{\prime}} \delta_{\boldsymbol{r}, \boldsymbol{r}^{\prime}}, \\
& \left\{\hat{\psi}_{\alpha, \boldsymbol{r}}^{\dagger}, \hat{\psi}_{\alpha^{\prime}, \boldsymbol{r}^{\prime}}^{\dagger}\right\}=\left\{\hat{\psi}_{\alpha, \boldsymbol{r}}, \hat{\psi}_{\alpha^{\prime}, \boldsymbol{r}^{\prime}}\right\}=0 .
\end{aligned}
$$

Hermiticity $\hat{H}=\hat{H}^{\dagger}$ is imposed by demanding that the single-particle matrix elements obey

$$
\mathcal{H}_{\alpha, \boldsymbol{r} ; \alpha^{\prime}, \boldsymbol{r}^{\prime}}=\mathcal{H}_{\alpha^{\prime}, \boldsymbol{r}^{\prime} ; \alpha, \boldsymbol{r}}^{*}
$$


Locality, in the orbital-lattice basis, is imposed by demanding that

$$
\lim _{\left|\boldsymbol{r}-\boldsymbol{r}^{\prime}\right| \rightarrow \infty}\left|\mathcal{H}_{\alpha, \boldsymbol{r} ; \alpha^{\prime}, \boldsymbol{r}^{\prime}}\right| \leqslant \lim _{\left|\boldsymbol{r}-\boldsymbol{r}^{\prime}\right| \rightarrow \infty} c \times e^{-\left|\boldsymbol{r}-\boldsymbol{r}^{\prime}\right| / \ell}
$$

for some positive constant $c$ and for some characteristic length scale $\ell$ independent of the lattice sites $\boldsymbol{r}, \boldsymbol{r}^{\prime}=1, \cdots, N$ and of the orbital indices $\alpha, \alpha^{\prime}=1, \cdots, N_{\text {orb }}$. If we collect the orbital $(\alpha)$ and lattice $(\boldsymbol{r})$ indices into a single collective index $I \equiv(\alpha, \boldsymbol{r})$, we may introduce the short-hand notation

$$
\hat{H} \equiv \sum_{I, I^{\prime} \in \Omega} \hat{\psi}_{I}^{\dagger} \mathcal{H}_{I I^{\prime}} \hat{\psi}_{I^{\prime}} \equiv \hat{\psi}^{\dagger} \mathcal{H} \hat{\psi}
$$

where $\mathcal{H}$ is a Hermitian $N_{\text {tot }} \times N_{\text {tot }}$ matrix with $N_{\text {tot }}=N_{\text {orb }} \times N$ and $\Omega$ is the set

$$
\Omega:=\left\{1, \cdots, N_{\text {orb }}\right\} \times \Lambda
$$

obtained by taking the Cartesian product of the set of orbitals with the set of lattice points.

The Hermitian matrix $\mathcal{H}$ has $N_{\text {tot }}$ pairs of eigenvalues and eigenvectors $\left(\varepsilon_{I}, v_{I}\right)$ that are defined by demanding that

$$
\mathcal{H} v_{I}=\varepsilon_{I} v_{I}, \quad v_{I}^{\dagger} v_{I^{\prime}}=\delta_{I, I^{\prime}}
$$

for $I, I^{\prime}=1, \cdots, N_{\text {tot }}$. With the help of the unitary matrix

$$
\mathcal{U}=\left(v_{1}, \ldots, v_{N_{\text {tot }}}\right),
$$

we may represent the single-particle Hamiltonian $\mathcal{H}$ as the diagonal matrix

$$
\mathcal{U}^{\dagger} \mathcal{H} \mathcal{U}=\operatorname{diag}\left(\varepsilon_{1}, \ldots, \varepsilon_{N_{\text {tot }}}\right)
$$

The canonical transformation

$$
\hat{\psi}_{I}=: \sum_{J=1}^{N_{\text {tot }}} \mathcal{U}_{I J} \hat{\chi}_{J}
$$

gives the representation

$$
\hat{H}=\sum_{I=1}^{N_{\text {tot }}} \hat{\chi}_{I}^{\dagger} \varepsilon_{I} \hat{\chi}_{I} .
$$

The ground state of $N_{\mathrm{f}}$ fermions is then the Fermi sea

$$
\left|\Psi_{\mathrm{FS}}\right\rangle:=\prod_{I=1}^{N_{\mathrm{f}}} \hat{\chi}_{I}^{\dagger}|0\rangle, \quad \hat{\chi}_{I}|0\rangle=0,
$$

whereby we have assumed that

$$
I<I^{\prime} \Longrightarrow \varepsilon_{I} \leqslant \varepsilon_{I^{\prime}} .
$$


2.1.2. Entanglement spectrum of the equal-time one-point correlation matrix We seek to partition the Fock space $\mathfrak{F}$, on which the non-interacting Hamiltonian $\hat{H}$ defined by Eq. (2.2) acts, into two Fock subspace, which we denote by $\mathfrak{F}_{A}$ and $\mathfrak{F}_{B}$, according to the tensorial decomposition

$$
\mathfrak{F}=\mathfrak{F}_{A} \otimes \mathfrak{F}_{B} .
$$

Two ingredients are necessary to define the subspaces $\mathfrak{F}_{A}$ and $\mathfrak{F}_{B}$. We need a state from $\mathfrak{F}$ that is a single Slater determinant. It is for this quality that we choose the ground state $(2.3)$. We need a basis, that we choose to be the orbital-lattice basis defined by the representation (2.2).

Following Ref. [29], we start from the equal-time one-point correlation function (matrix)

$$
\mathcal{C}_{I J}:=\left\langle\Psi_{\mathrm{FS}}\left|\hat{\psi}_{I}^{\dagger} \hat{\psi}_{J}\right| \Psi_{\mathrm{FS}}\right\rangle, \quad I, J=1, \cdots, N_{\mathrm{tot}} .
$$

Insertion of Eqs. (2.3) and (2.2d) delivers

$$
\mathcal{C}_{I J}=\sum_{I^{\prime}=1}^{N_{\mathrm{f}}} \mathcal{U}_{I I^{\prime}}^{*} \mathcal{U}_{J I^{\prime}} \equiv \sum_{I^{\prime}=1}^{N_{\mathrm{f}}}\left\langle v_{I^{\prime}} \mid I\right\rangle\left\langle J \mid v_{I^{\prime}}\right\rangle,
$$

where $I, J=1, \cdots, N_{\text {tot }}$. We define the $N_{\text {tot }} \times N_{\text {tot }}$ correlation matrix $\mathcal{C}$ by its matrix elements 2.6. One then verifies that

$$
\mathcal{C}^{\dagger}=\mathcal{C}, \quad \mathcal{C}^{2}=\mathcal{C},
$$

i.e., the correlation matrix $\mathcal{C}$ is a Hermitian projector. The last equality of Eq. 2.6 introduces the bra and ket notation of Dirac for the single-particle eigenstates of $\mathcal{H}$ defined by Eq. (2.2) to emphasize that this projector is nothing but the sum over all the single-particle eigenstates of $\mathcal{H}$ that are occupied in the ground state. Thus, all $N_{\text {tot }}$ eigenvalues of the correlation matrix $\mathcal{C}$ are either the numbers 0 or 1 . When it is convenient to shift the eigenvalues of the correlation matrix from the numbers 0 or 1 to the numbers \pm 1 , this is achieved through the linear transformation

$$
\mathcal{Q}_{I J}:=\mathbb{I}-2 \mathcal{C}_{I J}, \quad I, J=1, \cdots, N_{\text {tot }} .
$$

The occupied single-particle eigenstates of $\mathcal{H}$ in the ground state of $\hat{H}$ are all eigenstates of $\mathcal{Q}$ with eigenvalue -1 . The unoccupied single-particle eigenstates of $\mathcal{H}$ in the ground state of $\hat{H}$ are all eigenstates of $\mathcal{Q}$ with eigenvalue +1 . In other words, $\mathcal{Q}$ is the difference between the projector onto the unoccupied single-particle eigenstates of $\mathcal{H}$ in the ground state of $\hat{H}$ and the projector onto the occupied single-particle eigenstates of $\mathcal{H}$ in the ground state of $\hat{H}$. As such $\mathcal{Q}$ possesses all the symmetries of $\mathcal{H}$ and all the spectral symmetries of $\mathcal{H}$.

We denote the $N_{\text {tot }}$-dimensional single-particle Hilbert space on which the correlation function $\mathcal{C}$ acts by $\mathfrak{H}$. The labels $A$ and $B$ for the partition are introduced through the direct sum decomposition

$$
\mathfrak{H}=\mathfrak{H}_{A} \oplus \mathfrak{H}_{B},
$$

whereby $A$ and $B$ are two non-intersecting subsets of the set $\Omega$ defined by Eq. (2.1f) such that

$$
\Omega=A \cup B, \quad A \cap B=\emptyset,
$$


and

$$
\mathcal{C}=\left(\begin{array}{cc}
C_{A} & C_{A B} \\
C_{A B}^{\dagger} & C_{B}
\end{array}\right), \quad \mathcal{Q}=\left(\begin{array}{cc}
\mathbb{I}-2 C_{A} & -2 C_{A B} \\
-2 C_{A B}^{\dagger} & \mathbb{I}-2 C_{B}
\end{array}\right) .
$$

By construction, the $N_{A} \times N_{A}$ block $C_{A}$ and the $N_{B} \times N_{B}$ block $C_{B}$ are Hermitian matrices. These blocks inherit the property that their eigenvalues are real numbers bounded between the numbers 0 and 1 from $\mathcal{C}$ being a Hermitian projector. We call the set

$$
\sigma\left(C_{A}\right):=\left\{\zeta_{\iota} \mid \exists v_{\iota} \in \mathbb{C}^{N_{\mathrm{A}}}, C_{A} v_{\iota}=\zeta_{\iota} v_{\iota}, \iota=1, \cdots, N_{\mathrm{A}}\right\}
$$

of single-particle eigenvalues of the block $C_{A}$ the entanglement spectrum of the correlation matrix $\mathcal{C}$. Any eigenvalue from $\sigma\left(C_{A}\right)$ obeys

$$
0 \leqslant \zeta_{\iota} \leqslant 1, \quad \iota=1, \cdots, N_{A}
$$

The single-particle eigenvalues of the $N_{A} \times N_{A}$ Hermitian matrix $C_{A}$ can be shifted from their support in the interval $[0,1]$ to the interval $[-1,+1]$ through the linear transformation

$$
Q_{A}:=\mathbb{I}-2 C_{A}
$$

We refer to the set $\sigma\left(Q_{A}\right)$ of single-particle eigenvalues of the $N_{A} \times N_{A}$ Hermitian matrix $Q_{A}$ as the entanglement spectrum of the correlation matrix $\mathcal{Q}$, which we shall abbreviate as the entanglement spectrum.

It is shown in Ref. [29] that there exists a $N_{A} \times N_{A}$ block Hermitian matrix (entanglement Hamiltonian) $H^{E} \equiv\left(H_{K, L}^{E}\right)_{K, L \in A}$ with the positive definite operator

$$
\hat{\rho}_{A}:=\frac{e^{-\sum_{K^{\prime}, L^{\prime} \in A} \hat{\psi}_{K^{\prime}}^{\dagger} H_{K^{\prime} L^{\prime}}^{E}, \hat{\psi}_{L^{\prime}}}}{\operatorname{tr}_{\mathfrak{F}_{A}} e^{-\sum_{K^{\prime}, L^{\prime} \in A} \hat{\psi}_{K^{\prime}}^{\dagger} H_{K^{\prime} L^{\prime}}^{E} \hat{\psi}_{L^{\prime}}}}
$$

whose domain of definition defines the Fock space $\mathfrak{F}_{A}$ such that the block $C_{A}$ from the correlation matrix $2.9 \mathrm{c})$ is

$$
C_{A}=\left(\operatorname{tr}_{\mathfrak{F}_{A}} \hat{\rho}_{A} \hat{\psi}_{K}^{\dagger} \hat{\psi}_{L}\right)_{K, L \in A}
$$

The positive definite matrix $\hat{\rho}_{A}$ is the reduced density matrix acting on the Fock space $\mathfrak{F}_{A}$ obtained by tracing the degrees of freedom from the Fock space $\mathfrak{F}_{B}$ in the density matrix

$$
\hat{\rho}_{\mathrm{FS}}:=\left|\Psi_{\mathrm{FS}}\right\rangle\left\langle\Psi_{\mathrm{FS}}\right|
$$

whose domain of definition is the Fock space $\mathfrak{F}$.

It is also shown in Ref. [29] that the single-particle spectrum $\sigma\left(C_{A}\right)$ of the $N_{A} \times N_{A}$ Hermitian matrix $C_{A}$ is related to the single-particle spectrum $\sigma(H)$ of the $N_{A} \times N_{A}$ Hermitian matrix $H^{E}$ by

$$
\zeta_{\iota}=\frac{1}{e^{\varpi}+1}, \quad \iota=1, \cdots, N_{A}
$$

Equation 2.13 states that the dependence of the eigenvalue $\zeta_{\iota}$ of $C_{A}$ on the eigenvalue $\varpi_{\iota}$ of $H$ is the same as that of the Fermi-Dirac function on the single-particle energy $\varpi_{\iota}$ when 
the inverse temperature is unity and the chemical potential is vanishing in units for which the Boltzmann constant is unity. Equation 2.13 is a one-to-one mapping between $\sigma\left(C_{A}\right)$ and $\sigma(H)$.

Equation (2.13) allows to express the entanglement entropy

$$
S_{A}^{\mathrm{ee}}:=-\operatorname{tr}_{\mathfrak{F}_{A}}\left(\hat{\rho}_{A} \ln \hat{\rho}_{A}\right)
$$

of the reduced density matrix $\hat{\rho}_{A}$ in terms of $\sigma\left(C_{A}\right)$ through

$$
S_{A}^{\mathrm{ee}}=-\sum_{\iota=1}^{N_{A}}\left[\zeta_{\iota} \ln \zeta_{\iota}+\left(1-\zeta_{\iota}\right) \ln \left(1-\zeta_{\iota}\right)\right]
$$

Zero modes of $C_{A}$ are eigenstates of $C_{A}$ with vanishing eigenvalues. The vector space spanned by the zero modes of $C_{A}$ is denoted by $\operatorname{ker}\left(C_{A}\right)$ in linear algebra. We conclude that the zero modes of $C_{A}$ do not contribute to the entanglement entropy.

2.1.3. Equal-time one-point correlation matrix and SUSY QM It was observed in Refs. [12] and [13] that the $2 \times 2$ block structure (2.9c) on the Hermitian correlation matrix $\mathcal{C}$ defined by its matrix elements 2.5$)$ is compatible with the condition $(2.7)$ that the correlation matrix is a projector if and only if the four conditions

$$
\begin{aligned}
& C_{A}^{2}-C_{A}=-C_{A B} C_{A B}^{\dagger}, \\
& Q_{A} C_{A B}=-C_{A B} Q_{B}, \\
& C_{A B}^{\dagger} Q_{A}=-Q_{B} C_{A B}^{\dagger}, \\
& C_{B}^{2}-C_{B}=-C_{A B}^{\dagger} C_{A B},
\end{aligned}
$$

hold. Here, the $N_{A} \times N_{A}$ matrix $Q_{A}$ was defined in Eq. (2.11) and we have introduced the $N_{B} \times N_{B}$ matrix $Q_{B}:=\mathbb{I}-2 C_{B}$.

The family of $2 N_{\text {susy }}+1$ operators

$$
\left(\widehat{\mathcal{Q}}_{1}^{\dagger}, \widehat{\mathcal{Q}}_{1}, \cdots, \widehat{\mathcal{Q}}_{N_{\text {susy }}}^{\dagger}, \widehat{\mathcal{Q}}_{N_{\text {susy }}}, \widehat{\mathcal{H}}\right)
$$

acting on a common Hilbert space realizes the graded Lie algebra of supersymmetric quantum mechanics (SUSY QM) if and only if

$$
\begin{aligned}
& {\left[\widehat{\mathcal{Q}}_{i}^{\dagger}, \widehat{\mathcal{H}}\right]=\left[\widehat{\mathcal{Q}}_{i}, \widehat{\mathcal{H}}\right]=0,} \\
& \left\{\widehat{\mathcal{Q}}_{i}, \widehat{\mathcal{Q}}_{j}^{\dagger}\right\}=\delta_{i, j} \widehat{\mathcal{H}}
\end{aligned}
$$

holds for $i, j=1, \cdots, N_{\text {susy }}$. SUSY QM is realized when $\widehat{\mathcal{H}}$, which is Hermitian by construction because of Eq. (2.17c), can be identified with a Hamiltonian. Conversely, a Hamiltonian in quantum mechanics is supersymmetric if there exists a factorization of the form 2.17). It is then convention to call the operators $\widehat{\mathcal{Q}}_{i}^{\dagger}$ and $\widehat{\mathcal{Q}}_{i}$ with $i=1, \cdots, N_{\text {susy }}$ supercharges owing to Eq. 2.17b). We are going to show that the algebra (2.16) is an example of SUSY QM with $N_{\text {susy }}=1$ in disguise. 
To this end, we define the $N_{A} \times N_{A}, N_{B} \times N_{B}, N_{A} \times N_{B}$, and $N_{B} \times N_{A}$ block matrices

$$
\begin{aligned}
& S_{A}:=\mathbb{I}-Q_{A}^{2}, \quad S_{B}:=\mathbb{I}-Q_{B}^{2}, \\
& M^{+} \equiv M^{\dagger}:=2 C_{A B}, \quad M^{-} \equiv M:=2 C_{A B}^{\dagger},
\end{aligned}
$$

respectively. By construction, the spectrum of the Hermitian $N_{A} \times N_{A}$ matrix $S_{A}$ and that of the Hermitian $N_{B} \times N_{B}$ matrix $S_{B}$ belong to the interval $[0,1]$. One verifies with the help of Eq. 2.16 that

$$
\begin{aligned}
& M^{+} S_{B}-S_{A} M^{+}=0, \quad M^{-} S_{A}-S_{B} M^{-}=0, \\
& M^{+} M^{-}=S_{A}, \quad M^{-} M^{+}=S_{B} .
\end{aligned}
$$

We cannot close the graded Lie algebra (2.17) with the four block matrices $(2.18)$. However, we still have the possibility to define the triplet of $N_{\text {tot }} \times N_{\text {tot }}$ matrices

$$
\mathcal{S}_{\text {susy }}=\left(\begin{array}{cc}
S_{A} & 0 \\
0 & S_{B}
\end{array}\right), \quad \mathcal{Q}_{\text {susy }}:=\left(\begin{array}{cc}
0 & 0 \\
M & 0
\end{array}\right),
$$

and $\mathcal{Q}^{\dagger}$. One verifies that they satisfy the graded Lie algebra

$$
\begin{aligned}
& {\left[\mathcal{Q}_{\text {susy }}, \mathcal{S}_{\text {susy }}\right]=\left[\mathcal{Q}_{\text {susy }}^{\dagger}, \mathcal{S}_{\text {susy }}\right]=0} \\
& \left\{\mathcal{Q}_{\text {susy }}, \mathcal{Q}_{\text {susy }}\right\}=\left\{\mathcal{Q}_{\text {susy }}^{\dagger}, \mathcal{Q}_{\text {susy }}^{\dagger}\right\}=0 \\
& \left\{\mathcal{Q}_{\text {susy }}, \mathcal{Q}_{\text {susy }}^{\dagger}\right\}=\mathcal{S}_{\text {susy }}
\end{aligned}
$$

i.e., the pair of supercharge matrices $\mathcal{Q}_{\text {susy }}^{\dagger}$ and $\mathcal{Q}_{\text {susy }}$ and the Hamiltonian matrix $\mathcal{S}_{\text {susy }}$ realize SUSY QM with $N_{\text {tot }}=1$.

The grading labeled by $A$ and $B$ in the definition of the matrices in Eq. 2.21) originates from the decomposition of the single particle Hilbert space $\mathcal{H}$ into the direct sum (2.9a). The matrix $\mathcal{S}_{\text {susy }}$ is block diagonal, i.e., it does not mix the subspaces $\mathfrak{H}_{A}$ and $\mathfrak{H}_{B}$. The pair of matrices $\mathcal{Q}_{\text {susy }}^{\dagger}$ and $\mathcal{Q}_{\text {susy }}$ are off-diagonal with respect to the labels $A$ and $B$, i.e., they mix the subspaces $\mathfrak{H}_{A}$ and $\mathfrak{H}_{B}$. The matrix $M^{-} \equiv M$ maps $\mathfrak{H}_{A}$ into $\mathfrak{H}_{B}$. Its adjoint $M^{+} \equiv M^{\dagger}$ maps $\mathfrak{H}_{B}$ into $\mathfrak{H}_{A}$. In the context of SUSY QM, the pair $M^{-}$and $M^{+}$are called intertwiner.

We are now going to show that the Eqs. (2.18), 2.20), and 2.21) imply that the number of linearly independent eigenstates of $\mathcal{S}_{\text {susy }}$ with vanishing eigenvalues, i.e., the number of linearly independent zero modes is larger or equal to

$$
\left|\operatorname{dim} \mathfrak{H}_{A}-\operatorname{dim} \mathfrak{H}_{B}\right| \equiv\left|N_{A}-N_{B}\right|
$$

To see this, we assume without loss of generality that $N_{A}>N_{B}$. When the dimension $N_{A}$ of $\mathfrak{H}_{A}$ is larger than the dimension $N_{B}$ of $\mathfrak{H}_{B}, M^{+}$is a rectangular matrix with more rows than columns while $M^{-}$is a rectangular matrix with more columns than rows. There follows two consequences. On the one hand, the condition

$$
M^{-} \mathfrak{H}_{A}=0
$$


that defines the null space of $M^{-}$necessarily admits $N_{A}-N_{B}>0$ linearly independent solutions in $\mathfrak{H}_{A}$, for we must solve $N_{B}$ equations for $N_{A}$ unknowns. On the other hand, the condition

$$
M^{+} \mathfrak{H}_{B}=0
$$

that defines the null space of $M^{+}$is overdetermined, for we must solve $N_{A}$ equations for $N_{B}$ unknowns. The condition

$$
\mathcal{S}_{\text {susy }} \mathfrak{H}=0
$$

that defines the null space of $\mathcal{S}_{\text {susy }}$ delivers at least $N_{A}-N_{B}>0$ linearly independent solutions of the form

$$
v=\left(\begin{array}{c}
v_{A} \\
0
\end{array}\right)
$$

where

$$
M^{-} v_{A}=0 .
$$

As observed by Witten in Ref. [30], the number of zero modes plays an important role in SUSY QM. The number of zero modes of $\mathcal{S}_{\text {susy }}$ is given by the Witten index

$$
\Delta_{\mathrm{w}}:=\left|\operatorname{dim} \operatorname{ker}\left(M^{-}\right)-\operatorname{dim} \operatorname{ker}\left(M^{+}\right)\right| \geqslant\left|N_{A}-N_{B}\right| \text {. }
$$

The relevance of Eq. (2.28) to the entanglement spectrum of non-interacting fermions is the main result of $\mathrm{Sec}$.2.1.3.

2.1.4. Chiral symmetry of the entanglement spectrum $\sigma\left(Q_{A}\right)$ We have seen that the existence of zero modes in the spectrum $\sigma\left(\mathcal{S}_{\text {susy }}\right)$ of $\mathcal{S}_{\text {susy }}$ defined in Eq. 2.20 is guaranteed when the dimensionalities $N_{A}$ and $N_{B}$ of the single-particle Hilbert spaces $\mathfrak{H}_{A}$ and $\mathfrak{H}_{B}$, respectively, are unequal. We have shown that this property is a consequence of a hidden supersymmetry. When $N_{A}=N_{B}$, we cannot rely on SUSY QM to decide if $0 \in \sigma\left(\mathcal{S}_{\text {susy }}\right)$.

The non-interacting Hamiltonian $\hat{H}$ defined by Eq. (2.1a) in the orbital-lattice basis acts on the Fock space $\mathfrak{F}$. Let $\mathscr{O}$ denote an operation, i.e., an invertible mapping of time, of the lattice, of the orbital degrees of freedom, or of compositions thereof. We represent this operation by either a unitary or an anti-unitary transformation on the Fock space $\mathfrak{F}$. In turn, it suffices to specify how the creation and annihilation operators defined by their algebra (2.1b) transform under the operation $\mathscr{O}$ to represent $\mathscr{O}$ on the Fock space $\mathfrak{F}$.

For example, the transformation law

$$
\hat{\psi}_{I} \rightarrow \hat{O} \hat{\psi}_{I} \hat{O}^{\dagger} \equiv \sum_{I^{\prime}=1}^{N_{\text {tot }}} \mathcal{O}_{I^{\prime} I} \hat{\psi}_{I^{\prime}}, \quad I=1, \cdots, N_{\text {tot }}
$$

where $\mathcal{O}=\left(\mathcal{O}_{I J}\right) \in U\left(N_{\text {tot }}\right)$ is a unitary matrix, realizes in a unitary fashion the operation $\mathscr{O}$. The non-interacting Hamiltonian $\hat{H}$ defined in Eq. 2.1) and the correlation matrix $\mathcal{C}$ defined in Eq. (2.5) obey the transformation laws

$$
\hat{H} \rightarrow \hat{O} \hat{H} \hat{O}^{\dagger} \Rightarrow \mathcal{H}^{\top} \rightarrow \mathcal{O}^{\dagger} \mathcal{H}^{\top} \mathcal{O}
$$


and

$$
\mathcal{C} \rightarrow \mathcal{O}^{\dagger} \mathcal{C} \mathcal{O}
$$

respectively.

For comparison, the operations of time reversal $\left(\mathscr{O}_{\mathrm{tr}}, \hat{O}_{\mathrm{tr}}, \mathcal{O}_{\mathrm{tr}}\right)$ and charge conjugation (that exchanges particle and holes) $\left(\mathscr{O}_{\mathrm{ph}}, \hat{O}_{\mathrm{ph}}, \mathcal{O}_{\mathrm{ph}}\right)$ are anti-unitary transformations for which

$$
\mathcal{C} \rightarrow \mathcal{O}_{\text {tr }}^{\dagger} \mathcal{C} \mathcal{O}_{\text {tr }}, \quad \mathcal{O}_{\text {tr }} \equiv \mathcal{T}_{\text {tr }} K, \quad \mathcal{T}_{\text {tr }}^{-1}=\mathcal{T}_{\text {tr }}^{\dagger}
$$

and

$$
\mathcal{C} \rightarrow \mathcal{O}_{\mathrm{ph}}^{\dagger} \mathcal{C} \mathcal{O}_{\mathrm{ph}}, \quad \mathcal{O}_{\mathrm{ph}} \equiv \mathcal{T}_{\mathrm{ph}} K, \quad \mathcal{T}_{\mathrm{ph}}^{-1}=\mathcal{T}_{\mathrm{ph}}^{\dagger}
$$

respectively. Here, $K$ is the anti-linear operation of complex conjugation.

The non-interacting Hamiltonian $\hat{H}$ has $\mathscr{O}$ as a symmetry if and only if

$$
\hat{O} \hat{H} \hat{O}^{\dagger}=\hat{H} \Longleftrightarrow \mathcal{H}^{\top}=\mathcal{O}^{\dagger} \mathcal{H}^{\top} \mathcal{O} \text {. }
$$

Moreover, if we assume the transformation law

$$
\left|\Psi_{\mathrm{FS}}\right\rangle \rightarrow e^{\mathrm{i} \Theta}\left|\Psi_{\mathrm{FS}}\right\rangle, \quad 0 \leqslant \Theta<2 \pi
$$

for the ground state (2.3), i.e., if we assume that the ground state does not break spontaneously the symmetry (2.34), then

$$
\mathcal{C}=\mathcal{O}^{\dagger} \mathcal{C} \mathcal{O} \Longleftrightarrow \mathcal{Q}=\mathcal{O}^{\dagger} \mathcal{Q} \mathcal{O}
$$

We want to derive what effect condition 2.36a has on the entanglement spectrum $\sigma\left(Q_{A}\right)$, i.e., when the direct sum decompositions (2.9) and

$$
\mathcal{O}=\left(\begin{array}{cc}
O_{A} & O_{A B} \\
O_{B A} & O_{B}
\end{array}\right) \in U\left(N_{\text {tot }}\right)
$$

hold for two special cases.

First, we assume that

$$
\mathcal{O}=\left(\begin{array}{cc}
O_{A} & 0 \\
0 & O_{B}
\end{array}\right), \quad O_{A} \in U\left(N_{A}\right), \quad O_{B} \in U\left(N_{B}\right)
$$

This situation arises when the operation $\mathscr{O}$ is compatible with the partitioning encoded by the two subsets $A$ and $B$ of $\Omega$ in the sense that

$$
A=\mathscr{O} A, \quad B=\mathscr{O} B
$$

If so, condition 2.36 simplifies to

$$
\begin{aligned}
& Q_{A}=O_{A}^{\dagger} Q_{A} O_{A}, \\
& C_{A B}=O_{A}^{\dagger} C_{A B} O_{B}, \\
& Q_{B}=O_{B}^{\dagger} Q_{B} O_{B} .
\end{aligned}
$$


Thus, the $N_{A} \times N_{A}$ Hermitian block matrices $C_{A}$ and $Q_{A}=\mathbb{I}-2 C_{A}$ inherit the symmetry obeyed by the $N_{\text {tot }} \times N_{\text {tot }}$ Hermitian matrix $\mathcal{H}$ when Eq. (2.37) holds.

Second, we assume that

$$
N_{A}=N_{B}=N_{\text {tot }} / 2
$$

and

$$
\mathcal{O}=\left(\begin{array}{cc}
0 & O_{A B} \\
O_{B A} & 0
\end{array}\right), \quad O_{A B}, O_{B A} \in U\left(N_{\text {tot }} / 2\right) .
$$

This situation arises when the operation $\mathscr{O}$ interchange the two subsets $A$ and $B$ of $\Omega$,

$$
A=\mathscr{O} B, \quad B=\mathscr{O} A .
$$

If so, condition (2.36) simplifies to

$$
\begin{aligned}
& Q_{A}=O_{B A}^{\dagger} Q_{B} O_{B A}, \\
& C_{A B}=O_{B A}^{\dagger} C_{B A} O_{A B} \\
& Q_{B}=O_{A B}^{\dagger} Q_{A} O_{A B} .
\end{aligned}
$$

We are going to combine Eq. 2.40) with Eq. 2.16). Multiplication of Eq. 2.40a) from the right by Eq. 2.40b gives the relation

$$
Q_{A} C_{A B}=O_{B A}^{\dagger} Q_{B} C_{B A} O_{A B},
$$

while multiplication of Eq. (2.40b) from the right by Eq. (2.40c) gives the relation

$$
C_{A B} Q_{B}=O_{B A}^{\dagger} C_{B A} Q_{A} O_{A B}
$$

We may then use Eq. (2.16b) to infer that

$$
Q_{A} C_{A B}=-C_{A B} Q_{B}
$$

A second use of Eq. (2.40c) delivers

$$
Q_{A} C_{A B}=-C_{A B} O_{A B}^{\dagger} Q_{A} O_{A B}
$$

We introduce the auxiliary $\left(N_{\text {tot }} / 2\right) \times\left(N_{\text {tot }} / 2\right)$ matrix

$$
\Gamma_{\mathscr{O} A}:=C_{A B} O_{A B}^{\dagger},
$$

whose domain and co-domain are the single-particle Hilbert space $\mathfrak{H}_{A}$. We may then rewrite Eq. (2.44) as the vanishing anti-commutator

$$
\left\{Q_{A}, \Gamma_{\mathscr{O} A}\right\}=0
$$

The same manipulations with the substitution $A \leftrightarrow B$ deliver

$$
\left\{Q_{B}, \Gamma_{\mathscr{O} B}\right\}=0
$$


where

$$
\Gamma_{\mathscr{O} B}:=C_{B A} O_{B A}^{\dagger},
$$

Equations 2.45b) and 2.45c) are the main result of Sec. 2.1.4.

An important consequence of Eq. 2.45b) is that the spectra $\sigma\left(Q_{A}\right)$ and $\sigma\left(Q_{B}\right)$, are endowed with a symmetry not necessarily present in the spectrum $\sigma(\mathcal{H})$ when Eqs. 2.34, 2.36, and 2.39) hold. This spectral symmetry is reminiscent of the so-called chiral symmetry, the property that a single-particle Hamiltonian anti-commutes with a unitary operator, although, here, the operator $\Gamma_{\mathscr{O}}$ is not necessarily unitary. If we assume that $C_{A B}$ is an invertible $\left(N_{\text {tot }} / 2\right) \times\left(N_{\text {tot }} / 2\right)$ matrix, to any pair $\left(1-2 \zeta_{\iota} \neq 0, v_{\iota}\right)$ that belongs to $\sigma\left(Q_{A}\right)$, the image pair $\left(-1+2 \zeta_{\iota} \neq 0, \Gamma_{\mathscr{O}} v_{\iota}\right)$ also belongs to $\sigma\left(Q_{A}\right)$, and conversely. We shall say that the entanglement spectrum $\sigma\left(Q_{A}\right)$ is chiral symmetric, when Eqs. 2.34), 2.36), (2.39) hold with $\Gamma_{\mathscr{O}}$ defined in Eq. (2.45d) invertible.

In this paper, we shall consider a regular $d$-dimensional lattice $\Lambda$, say a Bravais lattice. We denote with $P_{\|} \subset \mathbb{R}^{d}$ the plane with the coordinates

$$
\left(\begin{array}{c}
\boldsymbol{r}_{\|} \\
\mathbf{0}
\end{array}\right), \quad \boldsymbol{r}_{\|} \in \mathbb{R}^{n}, \quad n=1, \cdots, d-1
$$

We denote with $P_{\perp} \subset \mathbb{R}^{d}$ the plane with the coordinates

$$
\left(\begin{array}{c}
\mathbf{0} \\
\boldsymbol{r}_{\perp}
\end{array}\right), \quad \boldsymbol{r}_{\perp} \in \mathbb{R}^{d-n}, \quad n=1, \cdots, d-1 .
$$

Any lattice point $\boldsymbol{r}$ from $\Lambda$ can be written as

$$
\boldsymbol{r} \equiv\left(\begin{array}{c}
\boldsymbol{r}_{\|} \\
\boldsymbol{r}_{\perp}
\end{array}\right) \in \mathbb{R}^{d}
$$

We define the operation of reflection $\mathscr{R}$ about the plane $P_{\perp} \subset \mathbb{R}^{d}$ by

$$
\begin{aligned}
& \boldsymbol{r} \equiv\left(\begin{array}{c}
\boldsymbol{r}_{\|} \\
\boldsymbol{r}_{\perp}
\end{array}\right) \rightarrow \mathscr{R} \boldsymbol{r}:=\left(\begin{array}{c}
-\boldsymbol{r}_{\|} \\
+\boldsymbol{r}_{\perp}
\end{array}\right) \\
& \hat{\psi}_{\alpha, \boldsymbol{r}} \rightarrow \hat{R} \hat{\psi}_{\alpha, \boldsymbol{r}} \hat{R}^{\dagger} \equiv \sum_{\beta=1}^{N_{\text {orb }}} \mathrm{R}_{\alpha \beta} \hat{\psi}_{\beta, \mathscr{R} \boldsymbol{r}},
\end{aligned}
$$

for any $\alpha=1, \cdots, N_{\text {orb }}$ and $\boldsymbol{r} \in \Lambda$. We define the operation of parity (inversion) $\mathscr{P}$ by

$$
\begin{aligned}
& \boldsymbol{r} \rightarrow \mathscr{P} \boldsymbol{r}:=-\boldsymbol{r} \\
& \hat{\psi}_{\alpha, \boldsymbol{r}} \rightarrow \hat{P} \hat{\psi}_{\alpha, \boldsymbol{r}} \hat{P}^{\dagger} \equiv \sum_{\beta=1}^{N_{\text {orb }}} \mathrm{P}_{\alpha \beta} \hat{\psi}_{\beta, \mathscr{P} \boldsymbol{r}},
\end{aligned}
$$

for any $\alpha=1, \cdots, N_{\text {orb }}$ and $\boldsymbol{r} \in \Lambda$. 
Even though we may impose on the non-interacting Hamiltonian (2.1) the conditions of translation and point-group symmetries when the lattice $\Lambda$ is regular, a partitioning (2.9) might break these symmetries, for it involves lattice degrees of freedom. We seek a partitioning that preserves translation invariance within the "plane" (2.46b) that is normal to the "plane" 2.46a). With this partition in mind, we first perform the Fourier transformations

$$
\mathcal{H}_{I, I^{\prime}}\left(\boldsymbol{k}_{\perp}\right):=\frac{1}{N_{\perp}} \sum_{\boldsymbol{r}_{\perp}, \boldsymbol{r}_{\perp}^{\prime}} e^{\mathrm{i} \boldsymbol{k}_{\perp} \cdot\left(\boldsymbol{r}_{\perp}-\boldsymbol{r}_{\perp}^{\prime}\right)} \mathcal{H}_{I, \boldsymbol{r}_{\perp} ; I^{\prime}, \boldsymbol{r}_{\perp}^{\prime}},
$$

and

$$
\mathcal{C}_{I, I^{\prime}}\left(\boldsymbol{k}_{\perp}\right):=\frac{1}{N_{\perp}} \sum_{\boldsymbol{r}_{\perp}, \boldsymbol{r}_{\perp}^{\prime}} e^{\mathrm{i} \boldsymbol{k}_{\perp} \cdot\left(\boldsymbol{r}_{\perp}-\boldsymbol{r}_{\perp}^{\prime}\right)} \mathcal{C}_{I, \boldsymbol{r}_{\perp} ; I^{\prime}, \boldsymbol{r}_{\perp}^{\prime}}
$$

on the single-particle Hamiltonian (2.1c) and equal-time one-point correlation matrix (2.5), respectively, where

$$
I \equiv\left(\alpha, \boldsymbol{r}_{\|}\right), \quad I^{\prime} \equiv\left(\alpha^{\prime}, \boldsymbol{r}_{\|}^{\prime}\right),
$$

and $N_{\perp}$ is the number of lattice sites in $\Lambda$ holding a suitably chosen $\boldsymbol{r}_{\|}$fixed. For some suitably chosen $\boldsymbol{r}_{\perp}$ held fixed, we may then define the non-intersecting partitioning of the set

$$
\Omega:=\left\{\left(\alpha, \boldsymbol{r}_{\|}\right) \mid \alpha=1, \cdots, N_{\text {orb }},\left(\begin{array}{c}
\boldsymbol{r}_{\|} \\
\boldsymbol{r}_{\perp}
\end{array}\right) \in \Lambda\right\}
$$

into

$$
A=\mathscr{R} B=\mathscr{P} B
$$

and

$$
B=\mathscr{R} A=\mathscr{P} A .
$$

The discussion in Sec 2.1.3 is now applicable for each $\boldsymbol{k}_{\perp}$ separately, i.e., Eq. 2.16 becomes

$$
\begin{aligned}
& C_{A}^{2}\left(\boldsymbol{k}_{\perp}\right)-C_{A}\left(\boldsymbol{k}_{\perp}\right)=-C_{A B}\left(\boldsymbol{k}_{\perp}\right) C_{A B}^{\dagger}\left(\boldsymbol{k}_{\perp}\right), \\
& Q_{A}\left(\boldsymbol{k}_{\perp}\right) C_{A B}\left(\boldsymbol{k}_{\perp}\right)=-C_{A B}\left(\boldsymbol{k}_{\perp}\right) Q_{B}\left(\boldsymbol{k}_{\perp}\right), \\
& C_{A B}^{\dagger}\left(\boldsymbol{k}_{\perp}\right) Q_{A}\left(\boldsymbol{k}_{\perp}\right)=-Q_{B}\left(\boldsymbol{k}_{\perp}\right) C_{A B}^{\dagger}\left(\boldsymbol{k}_{\perp}\right), \\
& C_{B}^{2}\left(\boldsymbol{k}_{\perp}\right)-C_{B}\left(\boldsymbol{k}_{\perp}\right)=-C_{A B}^{\dagger}\left(\boldsymbol{k}_{\perp}\right) C_{A B}\left(\boldsymbol{k}_{\perp}\right) .
\end{aligned}
$$

We impose the symmetry conditions

$$
\begin{aligned}
& \mathcal{R}^{\dagger} C\left(\boldsymbol{k}_{\perp}\right) \mathcal{R}=C\left(+\boldsymbol{k}_{\perp}\right), \\
& \mathcal{P}^{\dagger} C\left(\boldsymbol{k}_{\perp}\right) \mathcal{P}=C\left(-\boldsymbol{k}_{\perp}\right),
\end{aligned}
$$

where $\mathcal{R}$ and $\mathcal{P}$ are unitary representations of the actions of the operations of reflection $\mathscr{R}$ and parity (inversion) $\mathscr{P}$ on the labels $2.49 \mathrm{c}$ for each $\boldsymbol{k}_{\perp}$ separately, respectively. We assume that $N_{A}=N_{B}$, for which it is necessary that the dimensionality $d$ of the lattice is even with 
the pair of orthogonal planes spanned by $\boldsymbol{r}_{\|}$and $\boldsymbol{r}_{\perp}$ each $(d / 2)$-dimensional, respectively. We then conclude Sec. 2.1.4 with the identities

$$
\begin{aligned}
& \left\{Q_{A}\left(\boldsymbol{k}_{\perp}\right), \Gamma_{\mathscr{R}}\left(\boldsymbol{k}_{\perp}\right)\right\}=0, \\
& Q_{A}\left(\boldsymbol{k}_{\perp}\right) \Gamma_{\mathscr{P}}\left(\boldsymbol{k}_{\perp}\right)+\Gamma_{\mathscr{P}}\left(\boldsymbol{k}_{\perp}\right) Q_{A}\left(-\boldsymbol{k}_{\perp}\right)=0,
\end{aligned}
$$

where $\Gamma_{\mathscr{R}}\left(\boldsymbol{k}_{\perp}\right)$ and $\Gamma_{\mathscr{P}}\left(\boldsymbol{k}_{\perp}\right)$ are not assumed invertible for each $\boldsymbol{k}_{\perp}$ separately.

2.1.5. Equal-time one-point correlation matrix and $\mathscr{P} \mathscr{C} \mathscr{T}$ symmetry Any local quantum field theory with a Hermitian Hamiltonian for which Lorentz invariance is neither explicitly nor spontaneously broken must preserve the composition $(\mathscr{P} \mathscr{C} \mathscr{T})$ of parity $(\mathscr{P})$, charge conjugation $(\mathscr{C})$, and time-reversal $(\mathscr{T})$, even though neither $\mathscr{P}$, nor $\mathscr{C}$, nor $\mathscr{T}$ need to be separately conserved. [31] The $\mathscr{P} \mathscr{C} \mathscr{T}$ theorem implies the existence of antiparticles. It also implies that any composition of two out of the triplet of transformations $\mathscr{P}, \mathscr{C}$, and $\mathscr{T}$ is equivalent to the third. [ Both the $\mathscr{P}$ and the $\mathscr{C} \mathscr{P}$ symmetries are violated by the weak interactions. [32, 33, 34] Consequently, the $\mathscr{P} \mathscr{C} \mathscr{T}$ theorem predicts that the $\mathscr{T}$ symmetry is violated by the weak interactions. A direct observation for the violation of the $\mathscr{T}$ symmetry by the weak interaction has been reported in Ref. [35].]

The $\mathscr{P} \mathscr{C} \mathscr{T}$ theorem does not hold anymore after relaxing the condition of Lorentz invariance. The effective Hamiltonians used in condensed matter physics generically break Lorentz invariance. However, the extend to which $\mathscr{P}, \mathscr{C}$, and $\mathscr{T}$ are separately conserved is not to be decided as a matter of principle but depends on the material and the relevant energy scales in condensed matter physics. For example, the effects of the earth magnetic field or those of cosmic radiation are for most practical purposes irrelevant to the properties of materials in condensed matter physics. A non-relativistic counterpart to charge conjugation symmetry holds in a mean-field treatment of superconductivity. Inversion symmetry, the nonrelativistic counterpart to symmetry under parity, is common to many crystalline states of matter. The effective Hamiltonian of electrons in magnetically inert materials preserves timereversal symmetry in the absence of an external magnetic field. Conversely, the effective Hamiltonian of electrons in the presence of magnetic impurities in metals or if the crystalline host is magnetic break explicitly time-reversal symmetry.

It has become clear in the last eight years that the notion of symmetry protected phases of matter is useful both theoretically and experimentally in condensed matter physics. It has first lead to a classification for the possible insulating phases of electrons that are adiabatically connected to Slater determinants. [36, 37, 38, 39, 40, 41] Some of the predicted insulating phases have then been observed in suitable materials. For each insulating phase, there exists a non-interacting many-body Hamiltonian $\hat{H}$ that is smoothly connected to all Hamiltonians describing this insulating phase by local unitary transformations. [9] This classification depends on the dimensionality of space and on the presence or absence of the following three discrete (i.e., involutive) symmetries. There is the symmetry of $\hat{H}$ under time reversal $\hat{O}_{\text {tr }}$,

$$
\left[\hat{H}, \hat{O}_{\mathrm{tr}}\right]=0, \quad \hat{O}_{\mathrm{tr}}^{2}= \pm 1 .
$$


There is the spectral symmetry of $\hat{H}$ under an anti-unitary transformation (particle-hole) $\hat{O}_{\mathrm{ph}}$,

$$
\left\{\hat{H}, \hat{O}_{\mathrm{ph}}\right\}=0, \quad \hat{O}_{\mathrm{ph}}^{2}= \pm 1 .
$$

There is the spectral symmetry of $\hat{H}$ under a unitary transformation (chiral) $\hat{O}_{\text {ch }}$,

$$
\left\{\hat{H}, \hat{O}_{\mathrm{ch}}\right\}=0 .
$$

There are two distinct insulating phases characterized by the presence or absence of the chiral spectral symmetry when both time reversal and particle-hole symmetry are broken. There are another eight distinct insulating phases when both time reversal and particle-hole symmetry are satisfied. For a fixed dimension of space, five of the ten insulating phases support gapless extended boundary states in geometries with open boundaries. Moreover, these three discrete symmetries can be augmented by discrete (involutive) symmetries that enforce reflection or mirror symmetries resulting in a rearrangement of which of the five insulating phases supporting gapless extended boundary states.

The single-particle matrix $\mathcal{Q}$ defined in Eq. (2.8) inherits from the single-particle matrix $\mathcal{H}$ defined by Eq. (2.2) any one of the three symmetries

$$
\begin{aligned}
& {\left[\mathcal{Q}, \mathcal{O}_{\mathrm{tr}}\right]=0, \quad \mathcal{O}_{\mathrm{tr}}^{2}= \pm 1,} \\
& \left\{\mathcal{Q}, \mathcal{O}_{\mathrm{ph}}\right\}=0, \quad \mathcal{O}_{\mathrm{ph}}^{2}= \pm 1, \\
& \left\{\mathcal{Q}, \mathcal{O}_{\mathrm{ch}}\right\}=0,
\end{aligned}
$$

with $\mathscr{O}_{\mathrm{ch}}$ implementing the chiral transformation and $\mathcal{O}_{\mathrm{ch}}$ its single-particle representation, $\mathscr{O}_{\mathrm{ph}}$ implementing the particle-hole transformation and $\mathcal{O}_{\mathrm{ph}}$ its single-particle representation, and $\mathscr{O}_{\mathrm{tr}}$ implementing reversal of time and $\mathcal{O}_{\mathrm{tr}}$ its single-particle representation, respectively. Here, we are assuming that there exists a partition

$$
\Omega=A \cup B, \quad A \cap B=\emptyset,
$$

that obeys

$$
A=\mathscr{O} A, \quad B=\mathscr{O} B,
$$

with $\mathscr{O}=\mathscr{O}_{\mathrm{tr}}, \mathscr{O}_{\mathrm{ph}}, \mathscr{O}_{\mathrm{ch}}$, when the time-reversal symmetry or the particle-hole or the chiral spectral symmetries of $\mathcal{Q}$ are inherited from those of $\mathcal{H}$.

Conservation of $\mathscr{P} \mathscr{C} \mathscr{T}$, as occurs in a relativistic local quantum-field theory, implies that the operations of parity $\mathscr{P}$, charge conjugation $\mathscr{C}$, and time-reversal $\mathscr{T}$ are not independent. The composition of any two of them is equivalent to the third one.

Even though $\mathscr{P} \mathscr{C} \mathscr{T}$ may not be conserved for the non-interacting Hamiltonian $\mathcal{H}$ defined in Eq. 2.2], its correlation matrix $\mathcal{Q}$ defined in Eq. (2.8) or its entanglement matrix $Q_{A}$ defined in Eq. 2.11) may obey a weaker form of a $\mathscr{P} \mathscr{C} \mathscr{T}$-like relation as we now illustrate by way of two examples.

Case when $\mathscr{P} \mathscr{C}$ is equivalent to $\mathscr{T}:$ When the parity (inversion) transformation $\mathscr{P}$ is a symmetry of $\mathcal{H}$, there exists a $\Gamma_{\mathscr{P} A}$ such that, by Eq. (2.45b), it anti-commutes with $Q_{A}$, i.e.,

$$
\left\{Q_{A}, \Gamma_{\mathscr{P} A}\right\}=0 .
$$


If we also assume that there exists a particle-hole transformation $O_{\mathrm{ph}}$ (an anti-unitary transformation) that anti-commutes with with $Q_{A}$ (e.g., if $\mathcal{H}$ is the Bogoliubov-de-Gennes Hamiltonian of a superconductor), i.e.,

$$
\left\{Q_{A}, O_{\mathrm{ph}}\right\}=0
$$

it then follows that the composition

$$
O_{\mathrm{tr}}:=O_{\mathrm{ph}} \Gamma_{\mathscr{P} A}
$$

is an anti-linear transformation that commutes with $Q_{A}$, i.e.,

$$
\left[Q_{A}, O_{\mathrm{tr}}\right]=0
$$

The symmetry of $\mathcal{H}$ under parity and the spectral symmetry of $\mathcal{H}$ under particle-hole transformation have delivered an effective anti-linear symmetry of $Q_{A}$.

Case when $\mathscr{C} \mathscr{T}$ is equivalent to $\mathscr{P}$ : We assume that $\mathcal{H}$ defined in Eq. (2.2) anticommutes with the conjugation of charge $\mathscr{C}$ represented by $\mathcal{O}_{\mathrm{ph}}$, while it commutes with reversal of time $\mathscr{T}$ represented by $\mathcal{O}_{\text {tr }}$. We also assume that the partition 2.56d obeys Eq. $2.56 \mathrm{e}$ when $\mathscr{O}$ is $\mathscr{O}_{\mathrm{ph}}$ or $\mathcal{O}_{\text {tr }}$, i.e.,

$$
\mathcal{O}_{\mathrm{ph}}=\left(\begin{array}{cc}
O_{\mathrm{ph} A} & 0 \\
0 & O_{\mathrm{ph} B}
\end{array}\right), \quad \mathcal{O}_{\operatorname{tr}}=\left(\begin{array}{cc}
O_{\operatorname{tr} A} & 0 \\
0 & O_{\operatorname{tr} B}
\end{array}\right)
$$

We define

$$
\mathcal{O}_{\mathrm{ch}}:=\mathcal{O}_{\mathrm{ph}} \mathcal{O}_{\mathrm{tr}}, \quad \mathcal{O}_{\mathrm{ch}}=\left(\begin{array}{cc}
O_{\mathrm{ch} A} & 0 \\
0 & O_{\mathrm{ch} B}
\end{array}\right) .
$$

It follows that $\mathcal{O}_{\text {ch }}$ is unitary, obeys Eq. 2.56e, and anti-commutes with $\mathcal{H}$. Hence, we may interpret $\mathcal{O}_{\text {ch }}$ as an effective chiral transformation. The symmetries and spectral symmetries of $\mathcal{H}$ are passed on to $\mathcal{Q}$ defined in Eq. (2.8). In particular,

$$
\mathcal{O}_{\mathrm{ch}} \mathcal{Q}=-\mathcal{Q} \mathcal{O}_{\mathrm{ch}}
$$

We make the additional assumption that $\mathfrak{h}_{A}$ and $\mathfrak{h}_{B}$ are isomorphic and that the action of $O_{\operatorname{ch} A}$ on $\mathfrak{h}_{A}$ is isomorphic to that of $O_{\operatorname{ch} B}$ on $\mathfrak{h}_{B}$. If so, we write

$$
O_{\mathrm{ch} A} \cong O_{\mathrm{ch} B} \cong O_{\mathrm{ch}}, \quad \mathcal{O}_{\mathrm{ch}}=\left(\begin{array}{cc}
O_{\mathrm{ch}} & 0 \\
0 & O_{\mathrm{ch}}
\end{array}\right)
$$

Equation 2.63 then gives four conditions,

$$
\begin{aligned}
& O_{\mathrm{ch}} Q_{A}=-Q_{A} O_{\mathrm{ch}}, \\
& O_{\mathrm{ch}} C_{A B}=-C_{A B} O_{\mathrm{ch}}, \\
& O_{\mathrm{ch}} C_{B A}=-C_{B A} O_{\mathrm{ch}}, \\
& O_{\mathrm{ch}} Q_{B}=-Q_{B} O_{\mathrm{ch}} .
\end{aligned}
$$


With the help of Eq. (2.16), one verifies that

$$
\begin{aligned}
& \left(O_{\mathrm{ch}} C_{B A}\right) Q_{A}=-O_{\mathrm{ch}} Q_{B} C_{B A}=Q_{B}\left(O_{\mathrm{ch}} C_{B A}\right), \\
& \left(O_{\mathrm{ch}} C_{B A}\right) C_{A B}=C_{B A}\left(C_{A B} O_{\mathrm{ch}}\right), \\
& \left(C_{A B} O_{\mathrm{ch}}\right) C_{B A}=C_{A B}\left(O_{\mathrm{ch}} C_{B A}\right), \\
& \left(C_{A B} O_{\mathrm{ch}}\right) Q_{B}=-C_{A B} Q_{B} O_{\mathrm{ch}}=Q_{A}\left(C_{A B} O_{\mathrm{ch}}\right) .
\end{aligned}
$$

This should be compared with Eq. (2.40). We conclude with the observation that there exists the transformation

$$
\mathcal{O}_{\text {eff }}:=\left(\begin{array}{cc}
0 & C_{A B} O_{\mathrm{ch}} \\
O_{\mathrm{ch}} C_{B A} & 0
\end{array}\right)
$$

that commutes with $\mathcal{Q}$,

$$
\left[\mathcal{Q}, \mathcal{O}_{\text {eff }}\right]=0
$$

The spectral symmetry of $\mathcal{H}$ under a particle-hole transformation and the symmetry of $\mathcal{H}$ under time reversal have conspired to provide $\mathcal{Q}$ with a symmetry under the transformation $\mathcal{O}_{\text {eff }}$.

\subsection{Interacting fermions}

We consider a many-body Hamiltonian acting on the Fock space $\mathfrak{F}$ introduced in Eq. (2.2) that describes $N_{\mathrm{f}}$ interacting fermions. Its normalized ground state is

$$
|\Psi\rangle:=\sum_{n=1}^{2^{N_{\text {tot }}}} \sum_{n_{1}^{(n)}=0,1} \cdots \sum_{n_{N_{\text {tot }}^{(n)}=0,1}} \delta_{n_{1}^{(n)}+\cdots+n_{N_{\text {tot }}}^{(n)}, N_{\mathrm{f}}} \times c_{n_{1}^{(n)}, \cdots, n_{N_{\text {tot }}}^{(n)}}^{(\Psi)}\left|n_{1}^{(n)}, \cdots, n_{N_{\text {tot }}}^{(n)}\right\rangle .
$$

The Slater determinant

$$
\left\langle n_{1}^{(n)}, \cdots, n_{N_{\text {tot }}}^{(n)}\right|:=\prod_{I=1}^{N_{\text {tot }}}\langle 0|\left(\hat{\chi}_{I}\right)^{n_{I}^{(n)}}
$$

has $c_{n_{1}^{(n)}, \cdots, n_{N_{\text {tot }}}^{(n)}}^{(\Psi)}$ as its overlap with the ground state $|\Psi\rangle$.

In the presence of interactions, the equal-time one-point correlation function (matrix)

$$
\mathcal{C}_{I J}:=\left\langle\Psi\left|\hat{\psi}_{I}^{\dagger} \hat{\psi}_{J}\right| \Psi\right\rangle, \quad I, J=1, \cdots, N_{\text {tot }},
$$

does not convey anymore the same information as the density matrix

$$
\hat{\rho}_{\Psi}:=|\Psi\rangle\langle\Psi|, \quad\langle\Psi \mid \Psi\rangle=1
$$

In particular, Eq. 2.70 does not encode the full information contained in the reduced density matrices

$$
\hat{\rho}_{\Psi}^{(A)}:=\operatorname{tr}_{\mathfrak{F}_{B}} \hat{\rho}_{\Psi}
$$


and

$$
\hat{\rho}_{\Psi}^{(B)}:=\operatorname{tr}_{\mathfrak{F}_{A}} \hat{\rho}_{\Psi},
$$

where we have defined the partition in Eqs. (2.4) and (2.9).

We seek a useful representation of the reduced density matrices $2.71 \mathrm{~b})$ and $(2.71 \mathrm{c})$ and how they might be related by symmetries of the interacting Hamiltonian. To this end, we assign the labels $\mu_{A}=1, \cdots, \operatorname{dim} \mathfrak{F}_{A}$ and $\mu_{B}=1, \cdots, \operatorname{dim} \mathfrak{F}_{B}$ to any orthonormal basis $\left\{\left|\Psi_{\mu_{A}}^{(A)}\right\rangle\right\}$ and $\left\{\left|\Psi_{\mu_{B}}^{(B)}\right\rangle\right\}$ that span the Fock spaces $\mathfrak{F}_{A}$ and $\mathfrak{F}_{B}$, respectively. Without loss of generality, we assume $\operatorname{dim} \mathfrak{F}_{A} \geqslant \operatorname{dim} \mathfrak{F}_{B}$. We may write the expansion

$$
|\Psi\rangle=\sum_{\mu_{A}=1}^{\operatorname{dim} \mathfrak{F}_{A}} \sum_{\mu_{B}=1}^{\operatorname{dim} \mathfrak{F}_{B}} D_{\mu_{A} \mu_{B}}\left|\Psi_{\mu_{A}}^{(A)}\right\rangle \otimes\left|\Psi_{\mu_{B}}^{(B)}\right\rangle
$$

with the overlaps $D_{\mu_{A} \mu_{B}} \in \mathbb{C}$ defining the matrix elements of the $\operatorname{dim} \mathfrak{F}_{A} \times \operatorname{dim} \mathfrak{F}_{B}$ matrix $D$.

At this stage, we make use of the singular value decomposition

$$
D=U \Sigma V^{\dagger}
$$

with

$$
U=\left(U_{\mu_{A} \mu_{A}^{\prime}}\right)
$$

a $\operatorname{dim} \mathfrak{F}_{A} \times \operatorname{dim} \mathfrak{F}_{A}$ unitary matrix,

$$
\Sigma=\left(\begin{array}{cccccc}
\sigma_{1} & 0 & \cdots & & & 0 \\
0 & \ddots & \ddots & & & \vdots \\
\vdots & \ddots & \sigma_{\mathfrak{R}} & & & \\
& & & 0 & \ddots & \\
& & & \ddots & \ddots & 0 \\
0 & & & & 0 & 0 \\
\vdots & & & & \vdots & \vdots
\end{array}\right), \quad 0<\sigma_{1} \leqslant \cdots \leqslant \sigma_{\mathfrak{R}}
$$

a $\operatorname{dim} \mathfrak{F}_{A} \times \operatorname{dim} \mathfrak{F}_{B}$ rectangular diagonal matrix of rank $\mathfrak{R} \leqslant \operatorname{dim} \mathfrak{F}_{B}$, and

$$
V=\left(V_{\mu_{B} \mu_{B}^{\prime}}\right)
$$

a $\operatorname{dim} \mathfrak{F}_{B} \times \operatorname{dim} \mathfrak{F}_{B}$ unitary matrix.

With the help of Eq. (2.73), we have the Schmidt decomposition:

$$
|\Psi\rangle=\sum_{\nu=1}^{\Re} \sigma_{\nu}\left|\widetilde{\Psi}_{\nu}^{(A)}\right\rangle \otimes\left|\widetilde{\Psi}_{\nu}^{(B)}\right\rangle
$$

where

$$
\left|\widetilde{\Psi}_{\nu}^{(A)}\right\rangle:=\sum_{\mu_{A}=1}^{\operatorname{dim} \mathfrak{F}_{A}} U_{\mu_{A} \nu}\left|\Psi_{\mu_{A}}^{(A)}\right\rangle
$$


and

$$
\left|\widetilde{\Psi}_{\nu}^{(B)}\right\rangle:=\sum_{\mu_{B}=1}^{\operatorname{dim} \mathfrak{F}_{B}} V_{\mu_{B}}^{*}\left|\Psi_{\mu_{B}}^{(B)}\right\rangle .
$$

The singular values $\left\{\sigma_{\nu}\right\} \equiv \sigma(\Sigma)$ of the rectangular matrix $\Sigma$ are non-negative and obey the normalization condition

$$
\sum_{\nu=1}^{\Re} \sigma_{\nu}^{2}=1
$$

owing to the facts that the ground state $(2.69)$ is normalized to one and that the basis of $\mathfrak{F}_{A}$ and $\mathfrak{F}_{B}$ are chosen orthonormal.

In the basis (2.74),

$$
\hat{\rho}_{\Psi}:=\sum_{\nu=1}^{\mathfrak{R}} \sum_{\nu^{\prime}=1}^{\mathfrak{R}} \sigma_{\nu} \sigma_{\nu^{\prime}}\left|\widetilde{\Psi}_{\nu}^{(A)}\right\rangle\left\langle\widetilde{\Psi}_{\nu^{\prime}}^{(A)}|\otimes| \widetilde{\Psi}_{\nu}^{(B)}\right\rangle\left\langle\widetilde{\Psi}_{\nu^{\prime}}^{(B)}\right|
$$

and the reduced density matrices $2.71 \mathrm{~b}$ ) and $2.71 \mathrm{c})$ become the spectral decompositions

$$
\hat{\rho}_{\Psi}^{(A)}=\sum_{\nu=1}^{\Re} \sigma_{\nu}^{2}\left|\widetilde{\Psi}_{\nu}^{(A)}\right\rangle\left\langle\widetilde{\Psi}_{\nu}^{(A)}\right|
$$

and

$$
\hat{\rho}_{\Psi}^{(B)}:=\sum_{\nu=1}^{\Re} \sigma_{\nu}^{2}\left|\widetilde{\Psi}_{\nu}^{(B)}\right\rangle\left\langle\widetilde{\Psi}_{\nu}^{(B)}\right|
$$

respectively. The reduced density matrices $\hat{\rho}_{\Psi}^{(A)}$ and $\hat{\rho}_{\Psi}^{(B)}$ are explicitly positive semi-definite. They share the same non-vanishing eigenvalues

$$
0<\sigma_{\nu}^{2} \equiv e^{-\omega_{\nu}}, \quad 0 \leqslant \omega_{\nu}<\infty, \quad \nu=1, \cdots, \mathfrak{R}
$$

each of which can be interpreted as the probability for the ground state $(2.69)$ to be in the orthonormal basis state $\left|\widetilde{\Psi}_{\nu}^{(A)}\right\rangle \otimes\left|\widetilde{\Psi}_{\nu}^{(B)}\right\rangle$.

As we did with Eq. (2.39a), we assume that

$$
\operatorname{dim} \mathfrak{F}_{A}=\operatorname{dim} \mathfrak{F}_{B}=\mathfrak{D} .
$$

Hence, $\operatorname{dim} \mathfrak{F}_{A}$ and $\operatorname{dim} \mathfrak{F}_{B}$ are isomorphic. This means that $\hat{\Gamma}_{A B}: \mathfrak{F}_{B} \mapsto \mathfrak{F}_{A}$ defined by

$$
\hat{\Gamma}_{A B}:=\sum_{\nu=1}^{\mathfrak{D}}\left|\widetilde{\Psi}_{\nu}^{(A)}\right\rangle\left\langle\widetilde{\Psi}_{\nu}^{(B)}\right|
$$

and $\hat{\Gamma}_{B A}: \mathfrak{F}_{A} \mapsto \mathfrak{F}_{B}$ defined by

$$
\hat{\Gamma}_{B A}:=\sum_{\nu=1}^{\mathfrak{D}}\left|\widetilde{\Psi}_{\nu}^{(B)}\right\rangle\left\langle\widetilde{\Psi}_{\nu}^{(A)}\right|
$$


are linear one-to-one maps. One verifies that

$$
\begin{aligned}
& \hat{\Gamma}_{A B}^{-1}=\hat{\Gamma}_{B A}=\hat{\Gamma}_{A B}^{\dagger}, \\
& \hat{\rho}_{\Psi}^{(A)}=\hat{\Gamma}_{A B} \hat{\rho}_{\Psi}^{(B)} \hat{\Gamma}_{A B}^{\dagger}, \quad \hat{\rho}_{\Psi}^{(B)}=\hat{\Gamma}_{A B}^{\dagger} \hat{\rho}_{\Psi}^{(A)} \hat{\Gamma}_{A B} .
\end{aligned}
$$

We conclude Sec. 2.2 with a counterpart for interacting fermions of the spectral symmetry (2.45). We assume that the operation $\mathscr{O}$ under which the partition is interchanged,

$$
A=\mathscr{O} B, \quad B=\mathscr{O} A,
$$

is represented by the unitary map $\mathcal{O}: \mathfrak{F}_{A} \mapsto \mathfrak{F}_{B}$ defined either by

$$
\left|\widetilde{\Psi}_{\nu}^{(B)}\right\rangle_{\mathscr{O}}:=\sum_{\nu^{\prime}=1}^{\Re} \mathcal{O}_{\nu \nu^{\prime}}^{*}\left|\widetilde{\Psi}_{\nu^{\prime}}^{(A)}\right\rangle
$$

or by its inverse $\mathcal{O}^{\dagger}: \mathfrak{F}_{B} \mapsto \mathfrak{F}_{A}$ defined by

$$
\left|\widetilde{\Psi}_{\nu^{\prime}}^{(A)}\right\rangle=: \sum_{\nu^{\prime \prime}=1}^{\Re} \mathcal{O}_{\nu^{\prime \prime} \nu^{\prime}}\left|\widetilde{\Psi}_{\nu^{\prime \prime}}^{(B)}\right\rangle_{\mathscr{O}},
$$

whereby

$$
\sum_{\nu^{\prime}=1}^{\Re} \mathcal{O}_{\nu \nu^{\prime}}^{*} \mathcal{O}_{\nu^{\prime \prime} \nu^{\prime}}=\delta_{\nu, \nu^{\prime \prime}}
$$

with $\nu, \nu^{\prime}, \nu^{\prime \prime}=1, \cdots, \mathfrak{R}$.

By Eq. 2.75b,

$$
\left(\hat{\rho}_{\Psi}^{(A)}\right)_{\mathscr{O}}:=\sum_{\nu, \nu^{\prime}, \nu^{\prime \prime}=1}^{\Re} \sigma_{\nu}^{2} \mathcal{O}_{\nu^{\prime} \nu}^{*} \mathcal{O}_{\nu^{\prime \prime} \nu}\left|\widetilde{\Psi}_{\nu^{\prime}}^{(B)}\right\rangle_{\mathscr{O}} \mathcal{O}\left\langle\widetilde{\Psi}_{\nu^{\prime \prime}}^{(B)}\right| .
$$

If we impose the symmetry constraint

$$
\sum_{\nu=1}^{\Re} \sigma_{\nu}^{2} \mathcal{O}_{\nu^{\prime} \nu}^{*} \mathcal{O}_{\nu^{\prime \prime} \nu}= \begin{cases}\sigma_{\nu^{\prime}}^{2} \delta_{\nu^{\prime}, \nu^{\prime \prime}}, & \text { if } \nu^{\prime}=1, \cdots, \mathfrak{R}, \\ 0, & \text { otherwise },\end{cases}
$$

(i.e., $\mathcal{O}^{\dagger} \Sigma \mathcal{O}=\Sigma$ ), we conclude that

$$
\left(\hat{\rho}_{\Psi}^{(A)}\right)_{\mathscr{O}}=\sum_{\nu=1}^{\Re} \sigma_{\nu}^{2}\left|\widetilde{\Psi}_{\nu}^{(B)}\right\rangle_{\mathscr{O} \mathscr{O}}\left\langle\widetilde{\Psi}_{\nu}^{(B)}\right| \equiv \hat{\rho}_{\mathscr{O} \Psi}^{(B)}
$$

We can combine Eqs. (2.77) and (2.81) as follows. Given are the interacting Hamiltonian $\hat{H}$ acting on the Fock space $\mathfrak{F}$ defined in the orbital-lattice basis, the density matrix $\hat{\rho}_{\Psi}$ for the ground state $|\Psi\rangle$ of $\hat{H}$, the partition $\mathfrak{F}=\mathfrak{F}_{A} \otimes \mathfrak{F}_{B}$, and the reduced density matrix $\hat{\rho}_{\Psi}^{(A)}$ and $\hat{\rho}_{\Psi}^{(B)}$ acting on the Fock spaces $\mathfrak{F}_{A}$ and $\mathfrak{F}_{B}$ with $\operatorname{dim} \mathfrak{F}_{A}=\operatorname{dim} \mathfrak{F}_{B}$, respectively. Let $\hat{\Sigma}$ 
represent the action by conjugation on the space of operators of the matrix $2.73 \mathrm{c})$. Let $\hat{O}$ represent the action by conjugation on the space of operators of the matrix $\mathcal{O}$ with the matrix elements (2.79). Let

$$
\hat{\Xi}:=\hat{\Gamma}_{B A} \hat{O}
$$

and assume the symmetry

$$
\hat{O}^{\dagger} \hat{\Sigma} \hat{O}=\hat{\Sigma}
$$

The symmetry

$$
\hat{\rho}_{\Psi}^{(A)}=\hat{\Xi}^{\dagger} \hat{\rho}_{\Psi}^{(A)} \hat{\Xi}
$$

obeyed by the reduced density matrix then follows.

\section{Physical versus entangling boundaries, spectral gap, and locality}

Topological band insulators are insulators with two equivalent properties in the thermodynamic limit. If the discrete symmetry of time reversal, or the spectral symmetries of charge conjugation or chirality are imposed together with periodic boundary conditions, the bundle of Bloch states making up a ground state defines a quantized index, i.e., a number that does not change if the bundle of Bloch states is changed smoothly without violating the discrete symmetries or closing the band gap. The quantization of this index is a topological attribute of the occupied bands that is protected by certain discrete symmetries. If open boundary conditions are imposed instead of periodic ones, mid-gap single-particle states that are exponentially localized on the boundaries descend from the continuum of occupied and unoccupied Bloch states. These boundary states are protected, i.e., robust to perturbations that are compliant with the discrete symmetries, change smoothly Bloch states, and do not close the band gap. This equivalence between a topological index when periodic boundary conditions are imposed and protected boundary states when open boundary conditions are imposed is an example of a bulk-edge correspondence.

We shall call the boundaries in position space (i.e., in the orbital basis) that are selected by imposing open boundary conditions on the single-particle Hamiltonian $\mathcal{H}$ defined in Eq. (2.1) the physical boundaries. It is possible to define another class of boundaries in position space (i.e., in the orbital basis) by performing the partitioning (2.9). We call the boundaries that separate the lattice labels of the orbital basis in set $A$ from those in set $B$ the entangling boundaries. The number of entangling boundaries depends on whether periodic, open, or mixed boundary conditions are imposed. For a one-dimensional lattice such as the one shown in Fig. 2(a), choosing periodic boundary conditions selects no physical boundaries as is shown in the left panel of Fig. 2(b), while it selects two identical entangling boundaries as is shown in the left panel of Fig. 2(c). On the other hand, choosing open boundary conditions selects two identical physical boundaries as is shown in the right panel of Fig. 2(b), while it selects one physical boundary and one entangling boundary as is shown in the right panel of Fig. 2(c).

It has been proposed that the existence of protected single-particle states that are localized at the entangling boundaries, i.e., protected entangling boundary states, is a more refined signature for topological band insulators than the existence of protected physical boundary 
states. Indeed, it is argued that the existence of protected physical boundary states implies the existence of protected entangling boundary states for a suitable partition [3, 12, 8, 13]. However, the converse is known not to hold. For example, three-dimensional inversion symmetric topological insulators have been constructed such that the physical boundary states can be gapped out by inversion-symmetric perturbations, while the entangling boundary states remain gapless. [13, 12]

\subsection{Spectral gap and locality of the equal-time one-point correlation matrix}

In this context, the following observations are crucial. On the one hand, none of the results of Sec. 2 are sensitive to the presence or absence of a spectral gap $\Delta$ separating the Slater determinant entering the definition (2.5) of the equal-time one-point correlation matrix $\mathcal{C}$ from all excited states when periodic boundary conditions are imposed. On the other hand, the stability analysis from Secs. 4,7 only makes sense if $\Delta>0$, since the exponential decay of the boundary states away from the boundaries is controlled by the characteristic length $\xi \propto 1 / \Delta$. Consequently, the overlap of two boundary states localized on disconnected boundaries a distance $\propto N$ apart vanishes exponentially fast as the thermodynamic limit $N \rightarrow \infty$ is taken, i.e., the matrix elements $\mathcal{C}_{I J}$ in Eq. (2.5) also decay exponentially fast if the pair of repeat unit cells in the collective labels $I$ and $J$ are a distance larger than $\xi$ apart. In particular, the matrix elements entering the upper-right block $C_{A B}$ in Eq. (2.9) and those entering the transformation (2.45d) are suppressed exponentially in magnitude if they correspond to two repeat unit cells a distance larger than $\xi$ apart. It is only because of the presence of a spectral gap above the ground state of the Hamiltonian $\mathcal{H}$ defined in Eq. (2.1) that $\mathcal{C}$ defined in Eq. (2.9) inherits from $\mathcal{H}$ its locality in the orbital basis.

\subsection{Spectral gap and locality of the spectral symmetry (2.45d)}

In the remaining of this paper, we shall be concerned with exploring the effects of the spectral symmetry $(2.45 \mathrm{~d})$ on the entanglement spectra when a single-particle bulk spectral gap $\Delta$ guarantees that the equal-time one-point correlation matrices (2.5) and (2.8) are local for bulk-like separations, i.e., their matrix elements in the position basis can be bounded from above by the exponential factor $a \exp (-b|\boldsymbol{r}| \Delta)$ where $a$ and $b$ are some numerical factor of order unity and $|\boldsymbol{r}| \geq 1 / \Delta$ (in units with the Planck constant set to $\hbar=1$ and the speed of light set to $c=1$ ) is the bulk-like distance in space of the lattice degrees of freedom entering the bra and ket. In other words, we seek to understand how the spectral properties of the block $Q_{A}$ of the equal-time one-point correlation matrix defined in Eq. (2.8) are affected by the assumption that a symmetry operation $\mathscr{O}$ conspires with the partition of the degrees of freedom of the single-particle Hilbert space so as to obey Eq. (2.39), i.e., the map

$$
\Gamma_{\mathscr{O} A}: \mathfrak{H}_{A} \mapsto \mathfrak{H}_{A}
$$

defined by the matrix multiplication $\Gamma_{\mathscr{O} A}:=C_{A B} O_{A B}^{\dagger}$ anti-commutes with $Q_{A}$.

Typically, the symmetry operation $\mathscr{O}$ is a point-group symmetry, say an inversion or reflection symmetry. Hence, we shall assume that the symmetry operation $\mathscr{O}$ is not local. 


\section{(a)}

$A$

\begin{abstract}
$\mathrm{x}=0$
\end{abstract}
$B$

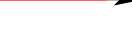

(b)

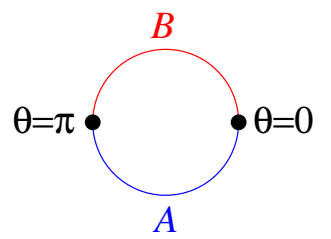

Figure 1. (Color online) (a) The real line $\{x \in \mathbb{R}\}$ is partitioned into two open sets, the negative axis $A$ and the positive axis $B$. The boundary between $A$ and $B$ is the origin at $x=0$, a compact set. This boundary is the entangling boundary of the real line, a connected set. The real line has two disconnected physical boundaries at $x=-\infty$ and $x=+\infty$. The inversion about the origin $x \mapsto-x$ is smooth, exchanges $A$ and $B$, and has the entangling boundary as its unique fixed point. The map $x \mapsto-1 / x$ is not smooth at the origin, exchanges $A$ and $B$, and has no fixed point. The real line is the limit $r \rightarrow 0$ of a cylinder with the radius $r$ embedded in three-dimensional Cartesian space. (b) The circle $\{\theta \in[0,2 \pi[\}$ can be interpreted as the compactification of the real line if the physical boundaries at $x=-\infty$ and $x=+\infty$ are identified with the angle $\theta=\pi$ and the origin of the real line is identified as the angle $\theta=0$. In doing so, the partition into the open sets $A$ and $B$ of the real line acquires two entangling boundaries at $\theta=0$ and $\theta=\pi$, respectively. These are two disconnected compact sets. The inversion about $\theta=0$ defined by $\theta \mapsto-\theta$ is smooth, exchanges $A$ and $B$, and has the two distinct fixed points $\theta=0$ and $\theta=\pi$. The inversion about $\theta=\pi$ defined by $\theta \mapsto \pi-\theta$ is smooth, leaves $A(B)$ invariant as a set, and exchanges $\theta=0$ and $\theta=\pi$. The translation defined by $\theta \mapsto \pi+\theta$, the composition of the two previous inversions, is smooth, exchanges $A$ and $B$, and has no fixed points. A circle of radius $R$ is the limit $r \rightarrow 0$ of a ring torus obtained by revolving a circle of radius $r$ in three-dimensional Cartesian space about an axis coplanar with the circle a distance $R>r$ apart from the center of mass of the revolving circle.

The spectral symmetry of $Q_{A}$ generated by $\Gamma_{\mathscr{O} A}$ allows to draw definitive conclusions on the existence of protected zero modes in the entanglement spectrum $\sigma\left(Q_{A}\right)$ only if $\Gamma_{\mathscr{O} A}$ is a local operation for bulk-like separations. For this reason, we devote section (3.2) to studying the conditions under which $\Gamma_{\mathscr{O} A}$ is local for bulk-like separations.

We shall consider two geometries for simplicity. Either we impose on $d$-dimensional space the geometry of a cylinder by imposing periodic boundary conditions in all $(d-1)$ directions in space while imposing open boundary conditions for the last direction. Or we impose on $d$-dimensional space the geometry of a torus by imposing periodic boundary conditions to all $d$ directions in space. When space is one dimensional, the cases of cylindrical and torus geometry are illustrated in Fig. 1(a) and 1(b), respectively.

For a cylindrical geometry, as illustrated in Fig. 1(a), there are two disconnected compact physical boundaries at $x= \pm \infty$ and one compact entangling boundary at $x=0$, where $x$ is the non-compact coordinate along the cylinder axis. We choose the symmetry operation $\mathscr{O}$ to be the mirror operation $x \mapsto-x$ that leaves the compact entangling boundary at $x=0$ point-wise invariant, while it exchanges the two disconnected compact physical boundaries at $x= \pm \infty$. The matrix elements of $\Gamma_{\mathscr{O} A}$ involving bra and kets with lattice degrees of freedom a distance $|\boldsymbol{r}| \gg 1 / \Delta$ away from the entangling boundary at $x=0$ are exponentially suppressed by the factor $\exp (-b|\boldsymbol{r}| \Delta)$ originating from $C_{A B}^{\dagger}$. Hence, $\Gamma_{\mathscr{O} A}$ is a local operator for bulk-like separations that generates a spectral symmetry on $Q_{A}$ without mixing boundary 
states localized on disconnected boundaries (whether physical or entangling).

For a torus geometry, as illustrated in Fig. 11(a), there are two disconnected compact entangling boundaries at $\theta=0$ and $\theta=\pi$. On the one hand, we may choose the symmetry operation $\mathscr{O}$ to be the mirror operation $\theta \mapsto-\theta$ that leaves the compact entangling boundary at $\theta=0$ and $\theta=\pi$ point-wise invariant. The matrix elements of $\Gamma_{\mathscr{O} A}$ involving bra and kets with lattice degrees of freedom a distance $|\boldsymbol{r}| \gg 1 / \Delta$ away from the entangling boundaries at $\theta=0$ and $\theta=\pi$ are exponentially suppressed by the factor $\exp (-b|\boldsymbol{r}| \Delta)$ originating from $C_{A B}^{\dagger}$. Hence, $\Gamma_{\mathscr{O} A}$ is again a local operator for bulk-like separations that generates a spectral symmetry on $Q_{A}$ without mixing boundary states localized on disconnected entangling boundaries. On the other hand, we may choose the symmetry operation $\mathscr{O}$ to be the mirror operation $\theta \mapsto \pi+\theta$ that exchanges the compact entangling boundary at $\theta=0$ and $\theta=\pi$. Hence, $\Gamma_{\mathscr{O} A}$ is not a local operator for it mixes with an amplitude of order unity boundary states localized on disconnected entangling boundaries separated by an arbitrary distance. Hence, even though $\Gamma_{\mathscr{O} A}$ generates a spectral symmetry of the entanglement spectrum of $Q_{A}$, it cannot be used to deduce any stability properties of the zero modes localized on the boundaries.

Sections 477 are devoted to the stability analysis of entangling boundary states by way of examples in one and two dimensions. This stability analysis requires distinguishing the nature of the boundaries in the partition (2.9), for whether these boundaries are physical or entangling depends on the choice of the boundary conditions imposed along the $d$ dimensions of space, as we have illustrated for the case of $d=1$ in Fig. 2. This stability analysis also requires determining if the spectral symmetry $\Gamma_{\mathscr{O} A}$ is local or not, as we have illustrated for the case of $d=1$ in Fig. 1 .

\section{Topological insulator protected by reflection (inversion) symmetry in one dimension}

\subsection{Hamiltonian}

Our first example is defined by choosing $d=1$ and $N_{\text {orb }}=4$ in Eq. 2.1). The lattice $\Lambda$ is one-dimensional with the lattice spacing $2 \mathfrak{a}$. It is labeled by the integers $r=1, \cdots, N$. To represent the single-particle Hamiltonian with the matrix elements (2.1d), we introduce two sets of Pauli matrices. We associate to the unit $2 \times 2$ matrix $\sigma_{0}$ and the three Pauli matrices $\sigma_{1}, \sigma_{2}$, and $\sigma_{3}$ two geometrical shapes, a square or a circle, corresponding to the eigenvalues of $\sigma_{3}$. We associate to the unit $2 \times 2$ matrix $\tau_{0}$ and the three Pauli matrices $\tau_{1}, \tau_{2}$, and $\tau_{3}$ two colors, black or white, corresponding to the eigenvalues of $\tau_{3}$. We choose the representation

$$
\hat{\psi}_{r}^{\dagger} \equiv\left(\begin{array}{llll}
\hat{\psi}_{\mathbf{a} ; r}^{\dagger} & \left.\hat{\psi}_{\square ; r}^{\dagger} \quad \hat{\psi}_{\bullet ; r}^{\dagger} \quad \hat{\psi}_{o ; r}^{\dagger} .\right)
\end{array}\right)
$$

and impose periodic boundary conditions

$$
\hat{\psi}_{r+N}^{\dagger}=\hat{\psi}_{r}^{\dagger}, \quad r=1, \cdots, N .
$$


(a)

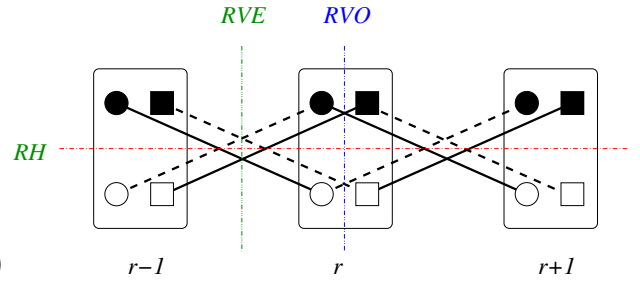

(b)
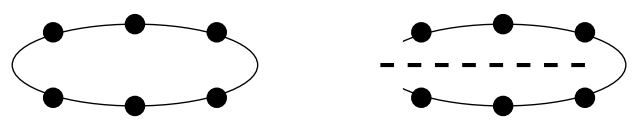

(c) $B$

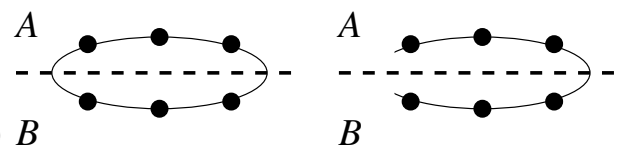

Figure 2. (Color online) (a) Hoppings of fermions between three consecutive sites $r-1, r$, and $r+1$ along a one-dimensional ring with four orbitals per site. A repeat unit cell is labeled by the integer $r=1, \cdots, N$ and is pictured by a rounded rectangular frame. A unit cell contains four orbitals that are pictured by black or white discs or squares, respectively. The hopping amplitude $t+\delta t \in \mathbb{R}$ is pictured by a full connecting line. The hopping amplitude $t-\delta t \in \mathbb{R}$ is pictured by a dashed connecting line. Hopping is only possible between orbitals of the same shape but distinct colors belonging to nearest-neighbor repeat unit cell. The figure is invariant under (i) the composition of the interchange of the full and dashed lines with the interchange of the black and white filling colors with a reflection about the horizontal dashone-dot (red) line $R H$ and (ii) the composition of the interchange of the circular and square shapes with a reflection about the vertical dash-two-dots (blue) line $R V O$ if $N$ is odd or the vertical dash-three-dots (green) line $R V E$ if $N$ is even. (b) Periodic boundary conditions are imposed (ring geometry) on the repeat unit cells represented by filled circles in the left panel, whereas open boundary conditions are imposed (open line geometry) on the repeat unit cells in the right panel. There are two physical boundaries on either sides of the cut represented by the dashed line a distance $N$ apart. (c) Periodic boundary conditions are imposed (ring geometry) on the repeat unit cells represented by filled circles in the left panel, whereas open boundary conditions are imposed (open line geometry) on the repeat unit cells in the right panel. The partitions $A$ and $B$ are made of the unit cells above and below the dashed line, respectively. There are two identical entangling boundaries an integer distance $N / 2$ apart in the left panel. There are two identical physical boundaries a distance $N$ apart in the right panel, each of which is an integer distance $N / 2$ apart from a single entangling boundary.

The non-interacting Hamiltonian is then defined by

$$
\begin{aligned}
\hat{H}:= & (t+\delta t) \sum_{r=1}^{N}\left(\hat{\psi}_{\square ; r+1}^{\dagger} \hat{\psi}_{\square ; r}+\hat{\psi}_{\circ ; r+1}^{\dagger} \hat{\psi}_{\bullet ; r}+\hat{\psi}_{\square ; r}^{\dagger} \hat{\psi}_{\square ; r+1}+\hat{\psi}_{\bullet ; r} \hat{\psi}_{\circ ; r+1}^{\dagger}\right) \\
& +(t-\delta t) \sum_{r=1}^{N}\left(\hat{\psi}_{\square ; r+1}^{\dagger} \hat{\psi}_{\square ; r}+\hat{\psi}_{\bullet ; r+1}^{\dagger} \hat{\psi}_{\circ ; r}+\hat{\psi}_{\square ; r} \hat{\psi}_{\square ; r+1}^{\dagger}+\hat{\psi}_{\circ ; r}^{\dagger} \hat{\psi}_{\bullet ; r+1}\right)
\end{aligned}
$$

in second quantization. It describes the nearest-neighbor hops of fermions with hopping amplitudes $t \pm \delta t \in \mathbb{R}$ as is illustrated in Fig. 2. The corresponding single-particle Hamiltonian 
can be written $\left[\tau_{ \pm}:=\left(\tau_{1} \pm \mathrm{i} \tau_{2}\right) / 2\right]$

$$
\begin{aligned}
\mathcal{H}_{r, r^{\prime}}(t, \delta t):=\delta_{r^{\prime}, r-1}\{ & (t+\delta t)\left[\frac{\sigma_{0}+\sigma_{3}}{2} \otimes \tau_{+}+\frac{\sigma_{0}-\sigma_{3}}{2} \otimes \tau_{-}\right] \\
& \left.+(t-\delta t)\left[\frac{\sigma_{0}+\sigma_{3}}{2} \otimes \tau_{-}+\frac{\sigma_{0}-\sigma_{3}}{2} \otimes \tau_{+}\right]\right\} \\
+\delta_{r^{\prime}, r+1} & \left\{(t+\delta t)\left[\frac{\sigma_{0}+\sigma_{3}}{2} \otimes \tau_{-}+\frac{\sigma_{0}-\sigma_{3}}{2} \otimes \tau_{+}\right]\right. \\
+ & \left.(t-\delta t)\left[\frac{\sigma_{0}+\sigma_{3}}{2} \otimes \tau_{+}+\frac{\sigma_{0}-\sigma_{3}}{2} \otimes \tau_{-}\right]\right\} .
\end{aligned}
$$

In the Bloch basis, the single-particle Hamiltonian (4.1e) becomes

$\mathcal{H}_{k, k^{\prime}}=\mathcal{H}_{k} \delta_{k, k^{\prime}}, \quad \mathcal{H}_{k}=2 t \cos k \sigma_{0} \otimes \tau_{1}-2 \delta t \sin k \sigma_{3} \otimes \tau_{2}, \quad k, k^{\prime}=-\pi+\frac{2 \pi}{N}, \cdots, \pi$,

with the single-particle spectrum consisting of the two-fold degenerate pair of bands

$$
\varepsilon_{ \pm ; k}= \pm 2|t| \sqrt{\cos ^{2} k+(\delta t / t)^{2} \sin ^{2} k} .
$$

Consequently, the band gap

$$
\Delta=4|\delta t|
$$

opens at the boundary of the first Brillouin zone for any non-vanishing $\delta t$.

\subsection{Symmetries}

The symmetries of the single-particle Hamiltonian defined by Eq. (4.1e) are the following, for any pair of sites $r, r^{\prime}=1, \cdots, N$.

[1] Translation symmetry holds,

$$
\mathcal{H}_{r, r^{\prime}}(t, \delta t)=\mathcal{H}_{r+n, r^{\prime}+n}(t, \delta t), \quad \forall n \in \mathbb{Z} .
$$

[2] When the dimerization vanishes

$$
\mathcal{H}_{r, r^{\prime}}(t, \delta t=0)=t\left(\delta_{r^{\prime}, r-1}+\delta_{r^{\prime}, r+1}\right) \sigma_{0} \otimes \tau_{1},
$$

the discrete symmetries

$$
0=\left[\mathcal{H}_{r, r^{\prime}}(t, \delta t=0), \sigma_{\mu} \otimes \tau_{\nu}\right]
$$

hold for $\mu=0,1,2,3$ and $\nu=0,1$, whereas the discrete spectral symmetries

$$
0=\left\{\mathcal{H}_{r, r^{\prime}}(t, \delta t=0), \sigma_{\mu} \otimes \tau_{\mu}\right\}
$$

hold for $\mu=0,1,2,3$ and $\nu=2,3$. 


\begin{tabular}{|c|c|c|c|c|c|c|c|c|c|c|c|c|c|c|c|c|c|c|}
\hline \multirow{2}{*}{$\mathcal{X}_{\mu \nu} \equiv \sigma_{\mu} \otimes \tau_{\nu}$} & \multicolumn{4}{|c|}{ Symmetry under $\mathscr{P}$} & \multicolumn{4}{|c|}{ Symmetry under $\mathscr{T}$} & \multicolumn{4}{|c|}{ Symmetry under $\mathscr{C}$} & \multicolumn{4}{|c|}{ Symmetry under $\mathscr{S}$} & \multirow{2}{*}{$\sigma\left(\widetilde{\mathcal{H}}_{\mu \nu}\right)$} & \multirow{2}{*}{$\sigma\left(\widetilde{Q}_{\mu \nu A}\right)$} \\
\hline & $\mathcal{P}_{01}$ & $\mathcal{P}_{10}$ & $\mathcal{P}_{20}$ & $\mathcal{P}_{31}$ & $\mathcal{T}_{00}$ & $\mathcal{T}_{11}$ & $\mathcal{T}_{21}$ & $\mathcal{T}_{30}$ & $\mathcal{C}_{03}$ & $\mathcal{C}_{12}$ & $\mathcal{C}_{22}$ & $\mathcal{C}_{33}$ & $\mathcal{S}_{03}$ & $\mathcal{S}_{12}$ & $\mathcal{S}_{22}$ & $\mathcal{S}_{33}$ & & \\
\hline $\mathcal{X}_{00}$ & $\circ$ & 0 & $\circ$ & $\circ$ & $\circ$ & $\circ$ & $\circ$ & $\circ$ & $x$ & $\times$ & $\times$ & $\times$ & $\times$ & $\times$ & $\times$ & $\times$ & $\times$ & $\circ$ \\
\hline $\mathcal{X}_{01}$ & ० & o & o & ० & $\circ$ & $\circ$ & o & $\circ$ & $\circ$ & $\circ$ & o & $\circ$ & $\circ$ & ० & o & o & $\circ$ & $\circ$ \\
\hline $\mathcal{X}_{02}$ & $x$ & 0 & 0 & $x$ & $x$ & $\circ$ & $\circ$ & $\times$ & $x$ & 0 & $\circ$ & $\times$ & 0 & $x$ & $\times$ & $\circ$ & $\circ$ & 0 \\
\hline $\mathcal{X}_{03}$ & $\times$ & $\circ$ & ○ & $\times$ & $\circ$ & $\times$ & $\times$ & $\circ$ & $\times$ & $\circ$ & $\circ$ & $\times$ & $\times$ & $\circ$ & $\circ$ & $\times$ & $x$ & $\times$ \\
\hline $\mathcal{X}_{10}$ & $\circ$ & 0 & $\times$ & $\times$ & 0 & 0 & $x$ & $\times$ & $\times$ & $x$ & 0 & $\circ$ & $x$ & $\times$ & 0 & 0 & $\circ$ & $\circ$ \\
\hline $\mathcal{X}_{11}$ & 0 & 0 & $x$ & $x$ & 0 & 0 & $\times$ & $\times$ & $\circ$ & 0 & $\times$ & $\times$ & 0 & 0 & $\times$ & $x$ & $x$ & 0 \\
\hline $\mathcal{X}_{12}$ & $\times$ & $\circ$ & $\times$ & $\circ$ & $\times$ & $\circ$ & $\times$ & $\circ$ & $\times$ & $\circ$ & $\times$ & $\circ$ & $\circ$ & $\times$ & $\circ$ & $\times$ & $\times$ & $\times$ \\
\hline $\mathcal{X}_{13}$ & $x$ & o & $x$ & o & 0 & $\times$ & $\circ$ & $\times$ & $x$ & 0 & $\times$ & 0 & $\times$ & 0 & $\times$ & 0 & 0 & $\circ$ \\
\hline $\mathcal{X}_{20}$ & 0 & $\times$ & 0 & $\times$ & $\times$ & 0 & $\times$ & 0 & 0 & $\times$ & 0 & $\times$ & $\times$ & 0 & $\times$ & 0 & 0 & 0 \\
\hline $\mathcal{X}_{21}$ & 0 & $\times$ & 0 & $x$ & $x$ & 0 & $x$ & 0 & $x$ & 0 & $\times$ & 0 & 0 & $\times$ & 0 & $x$ & $x$ & $\circ$ \\
\hline $\mathcal{X}_{22}$ & $\times$ & $\times$ & 0 & 0 & 0 & 0 & $\times$ & $\times$ & 0 & 0 & $\times$ & $\times$ & 0 & 0 & $\times$ & $\times$ & $\times$ & $\times$ \\
\hline $\mathcal{X}_{23}$ & $x$ & $x$ & 0 & $\circ$ & $x$ & $\times$ & $\circ$ & 0 & 0 & 0 & $x$ & $\times$ & $x$ & $\times$ & 0 & 0 & $\circ$ & $\circ$ \\
\hline $\mathcal{X}_{30}$ & 0 & $\times$ & $x$ & 0 & 0 & $x$ & $\times$ & 0 & $x$ & $\circ$ & $\circ$ & $\times$ & $\times$ & 0 & 0 & $x$ & $\times$ & 0 \\
\hline $\mathcal{X}_{31}$ & 0 & $\times$ & $\times$ & 0 & 0 & $x$ & $\times$ & 0 & 0 & $x$ & $x$ & $\circ$ & 0 & $\times$ & $x$ & 0 & 0 & 0 \\
\hline $\mathcal{X}_{32}$ & $x$ & $x$ & $x$ & $\times$ & $x$ & $\times$ & $\times$ & $\times$ & $x$ & $x$ & $\times$ & $\times$ & 0 & 0 & 0 & 0 & $\circ$ & 0 \\
\hline $\mathcal{X}_{33}$ & $x$ & $\times$ & $x$ & $x$ & 0 & 0 & 0 & 0 & $x$ & $\times$ & $x$ & $\times$ & $x$ & $\times$ & $x$ & $x$ & $x$ & $\times$ \\
\hline
\end{tabular}

Table 1. The spectrum $\sigma\left(\widetilde{\mathcal{H}}_{\mu \nu}\right)$ of the single-particle Hamiltonian $\widetilde{\mathcal{H}}_{\mu \nu}$ defined by Eq. 4.23 with open boundary conditions. Hamiltonian $\widetilde{\mathcal{H}}_{\mu \nu}$ is nothing but Hamiltonian $\mathcal{H}$ [defined in Eq. 4.1e]] perturbed additively by the term $\delta_{r, r^{\prime}}(t / 10) \mathcal{X}_{\mu \nu}$ with $\mathcal{X}_{\mu \nu} \equiv \sigma_{\mu} \otimes \tau_{\nu}$. The choices for $\mathcal{X}_{\mu \nu}$ made in the first eight rows enumerate all perturbations $\mathcal{V}_{\mu \nu}$ defined by Eq. 4.23b that enter Eq. 4.22), i.e., that preserve the symmetry under the parity of $\mathcal{H}$ generated by $\mathcal{P}_{10}$. The last two rows are two examples of a perturbation $\mathcal{V}_{\mu \nu}$ that breaks parity. The entanglement spectrum $\sigma\left(\widetilde{Q}_{\mu \nu A}\right)$ defined by Eq. 2.11 for the single-particle Hamiltonian $\widetilde{\mathcal{H}}_{\mu \nu}$ obeying periodic boundary conditions. The entry $\circ$ or $\times$ denotes the presence or the absence, respectively, of the symmetries under parity $\mathscr{P}$, charge conjugation $\mathscr{C}$, and time reversal $\mathscr{T}$ of the perturbation $\delta_{r, r^{\prime}}(t / 10) \mathcal{X}_{\mu \nu}$ for the first sixteen columns. In the last two columns, the entry $\circ$ or $\times$ denotes the presence or the absence, respectively, of zero modes (mid-gap) states in the spectra $\sigma\left(\widetilde{\mathcal{H}}_{\mu \nu}\right)$ and $\sigma\left(\widetilde{\mathcal{Q}}_{\mu \nu}\right)$ as determined by extrapolation to the thermodynamic limit of exact diagonalization with $N=12$.

[3] For any dimerization, the transformation laws

$$
\mathcal{H}_{r, r^{\prime}}(t,+\delta t)=\sigma_{\mu} \otimes \tau_{1} \mathcal{H}_{r, r^{\prime}}(t,-\delta t) \sigma_{\mu} \otimes \tau_{1}
$$

with $\mu=0,3$ implement the symmetry of Fig. 2 under the composition of the interchange of the full and dashed lines with the interchange of the black and white filling colors with a reflection about the horizontal dash-one-dot (red) line $R H$, whereas the transformation laws

$$
\mathcal{H}_{r, r^{\prime}}(t,+\delta t)=\sigma_{\mu} \otimes \tau_{1} \mathcal{H}_{r, r^{\prime}}(t,+\delta t) \sigma_{\mu} \otimes \tau_{1}
$$

hold for $\mu=1,2$ otherwise.

[4] Let $\mathscr{O}_{\mathrm{R}}$ be the operation that interchanges site $r_{1}$ with site $r_{N}$, site $r_{2}$ with site $r_{N-1}$, and so on, i.e., a reflection about the vertical dash-two-dots (blue) line $R V O$ if $N$ is odd or the vertical dash-three-dots (green) line $R V E$ if $N$ is even. For any dimerization, the transformation laws

$$
\mathcal{H}_{r, r^{\prime}}(t, \delta t)=+\sigma_{\mu} \otimes \tau_{0} \mathcal{H}_{\mathscr{O}_{\mathrm{R}} r, \mathscr{O}_{\mathrm{R}} r^{\prime}}(t, \delta t) \sigma_{\mu} \otimes \tau_{0}
$$


with $\mu=1,2$ are unitary symmetries, whereas the transformation laws

$$
\mathcal{H}_{r, r^{\prime}}(t, \delta t)=-\sigma_{\mu} \otimes \tau_{3} \mathcal{H}_{\mathscr{O}_{\mathrm{R}} r, \mathscr{O}_{\mathrm{R}} r^{\prime}}(t, \delta t) \sigma_{\mu} \otimes \tau_{3}
$$

with $\mu=1,2$ are unitary spectral symmetries.

It is also instructive to derive the symmetries and spectral symmetries of the singleparticle Hamiltonian 4.2 for any $k=\pi / N, \cdots, \pi$ from the first Brillouin zone.

To this end, it is convenient to introduce the more compact notation

$$
\mathcal{X}_{\mu \nu}:=\sigma_{\mu} \otimes \tau_{\nu}, \quad \mu, \nu=0,1,2,3
$$

for the sixteen linearly independent $4 \times 4$ Hermitian matrices that generate the unitary group $U(4)$. In the Bloch basis 4.2a),

$$
\mathcal{H}_{k, k^{\prime}}=\mathcal{H}_{k} \delta_{k, k^{\prime}}, \quad \mathcal{H}_{k}=2 t \cos k \mathcal{X}_{01}-2 \delta t \sin k \mathcal{X}_{32}
$$

for $k, k^{\prime}=\pi / N, \cdots, \pi$. We have taken advantage of the fact that $\mathcal{X}_{01}$ and $\mathcal{X}_{32}$ anti-commute to derive the band dispersions $4.2 \mathrm{~b}$ ).

There are eight matrices $\mathcal{X}_{\mu \nu}$ with $\mu=0,1,2,3$ and $\nu=0,1$ that commute with $\mathcal{X}_{01}$, there are eight matrices $\mathcal{X}_{\mu \nu}$ with $\mu=1,2$ and $\nu=1,3$ or $\mu=0,3$ and $\nu=0,2$ that commute with $\mathcal{X}_{32}$. This leaves the four matrices $\mathcal{X}_{00}, \mathcal{X}_{30}, \mathcal{X}_{11}$, and $\mathcal{X}_{21}$ that commute with $\mathcal{H}_{k}$ for all $k$ in the Brillouin zone.

There are eight matrices $\mathcal{X}_{\mu \nu}$ with $\mu=0,1,2,3$ and $\nu=2,3$ that anti-commute with $\mathcal{X}_{01}$, there are eight matrices $\mathcal{X}_{\mu \nu}$ with $\mu=1,2$ and $\nu=0,2$ or $\mu=0,3$ and $\nu=1,3$ that anticommute with $\mathcal{X}_{32}$. This leaves the four matrices $\mathcal{X}_{12}, \mathcal{X}_{22}, \mathcal{X}_{03}$, and $\mathcal{X}_{33}$ that anti-commute with $\mathcal{H}_{k}$ for all $k$ in the Brillouin zone.

The symmetries

$$
\begin{aligned}
\mathcal{O}_{\mathscr{P}}^{\dagger} \mathcal{H}_{-k} \mathcal{O}_{\mathscr{P}} & =\mathcal{H}_{+k}, \\
\mathcal{O}_{\mathscr{T}}^{\dagger} \mathcal{H}_{-k}^{*} \mathcal{O}_{\mathscr{T}} & =\mathcal{H}_{+k}
\end{aligned}
$$

with

$$
\begin{aligned}
& \mathcal{O}_{\mathscr{P}} \in\left\{\mathcal{X}_{01}, \mathcal{X}_{10}, \mathcal{X}_{20}, \mathcal{X}_{31}\right\} \\
& \mathcal{O}_{\mathscr{T}} \in\left\{\mathcal{X}_{00}, \mathcal{X}_{11}, \mathcal{X}_{21}, \mathcal{X}_{30}\right\}
\end{aligned}
$$

whereas the spectral symmetries

$$
\begin{aligned}
& \mathcal{O}_{\mathscr{C}}^{\dagger} \mathcal{H}_{-k}^{\top} \mathcal{O}_{\mathscr{C}}=-\mathcal{H}_{k}, \\
& \mathcal{O}_{\mathscr{S}}^{\dagger} \mathcal{H}_{k} \mathcal{O}_{\mathscr{S}}=-\mathcal{H}_{k},
\end{aligned}
$$

with

$$
\begin{aligned}
& \mathcal{O}_{\mathscr{C}} \in\left\{\mathcal{X}_{03}, \mathcal{X}_{12}, \mathcal{X}_{22}, \mathcal{X}_{33}\right\} \\
& \mathcal{O}_{\mathscr{S}} \in\left\{\mathcal{X}_{03}, \mathcal{X}_{12}, \mathcal{X}_{22}, \mathcal{X}_{33}\right\}
\end{aligned}
$$


follow. As anticipated, the set of chiral-like spectral symmetries is identical the set of particlehole-like spectral symmetries in view of the presence of four time-reversal-like symmetries. We shall use the notation $K$ for the anti-linear operation of complex conjugation. We shall also introduce the notation

$$
\begin{array}{lrl}
\text { Parity: } & \mathcal{P}_{10}:=\sigma_{1} \otimes \tau_{0}=+\mathcal{P}_{10}^{\top}, \\
\text { Charge Conjugation: } & \mathcal{C}_{03}:=\sigma_{0} \otimes \tau_{3} K=+\mathcal{C}_{03}^{\top}, \\
\text { Time Reversal: } & \mathcal{T}_{21}:=\sigma_{2} \otimes \tau_{1} K=-\mathcal{T}_{21}^{\top}, \\
\text { Chirality: } & \mathcal{S}_{22}:=\sigma_{2} \otimes \tau_{2}=+\mathcal{S}_{22}^{\top},
\end{array}
$$

say, to distinguish the operations of parity (reflection or inversion), charge conjugation (particle hole interchange), time reversal, and chirality, respectively. The symmetry under parity of $\mathcal{H}$ can be realized in four inequivalent ways. Correspondingly, we define the $4 \times 4$ matrices

$$
\text { Parity } \mathscr{P}: \quad \mathcal{P}_{01}, \mathcal{P}_{10}, \mathcal{P}_{20}, \mathcal{P}_{31} \text {, }
$$

that realize the algebra of the unit $2 \times 2$ matrix $\rho_{0}$ and of the three Pauli matrices $\rho_{1}, \rho_{2}$, and $\rho_{3}$. The symmetry under time reversal of $\mathcal{H}$ can be realized in four inequivalent ways. Correspondingly, we define the $4 \times 4$ matrices

$$
\text { Time Reversal } \mathscr{T}: \quad \mathcal{T}_{00}, \mathcal{T}_{11}, \mathcal{T}_{21}, \mathcal{T}_{30}
$$

that realize the algebra of $\rho_{0} K, \rho_{1} K, \rho_{2} K$, and $\rho_{3} K$. The symmetry under charge conjugation of $\mathcal{H}$ can be realized in four inequivalent ways. Correspondingly, we define the $4 \times 4$ matrices

$$
\text { Charge Conjugation } \mathscr{C}: \quad \mathcal{C}_{03}, \mathcal{C}_{12}, \mathcal{C}_{22}, \mathcal{C}_{33}
$$

that realize the algebra of $\rho_{0} K, \rho_{1} K, \rho_{2} K$, and $\rho_{3} K$. All the possible compositions of the operations for charge conjugation and time reversal give four realizations for the chiral symmetry of $\mathcal{H}$. Correspondingly, we define the $4 \times 4$ matrices

$$
\text { Chiral } \mathscr{S}: \quad \mathcal{S}_{03}, \mathcal{S}_{12}, \mathcal{S}_{22}, \mathcal{S}_{33}
$$

that realize the algebra of $\rho_{0}, \rho_{1}, \rho_{2}$, and $\rho_{3}$. There are sixteen columns in Table 11, each of which correspond to one of these matrix operations. We shall then select 12 triplets $\left(\mathcal{P}_{\mu \nu}, \mathcal{T}_{\mu \nu}, \mathcal{C}_{\mu \nu}\right)$ from Eqs. 4.13 to built the rows of Table 2 .

The single-particle Hamiltonian $\mathcal{H}$ is extremely sparse. This is reflected by it obeying the symmetries $\left(\mathcal{P}_{\mu}, \mathcal{T}_{\mu}, \mathcal{C}_{\mu}\right)$ with the pair $\mu$ and $\nu$ fixed by the columns from Table 1 . In particular, $\mathcal{H}$ cannot be assigned in a unique way the symmetry under time-reversal and the spectral symmetry under charge conjugation without additional informations of microscopic origin. Identifying the symmetric space generated by $\mathcal{H}$ is thus ambiguous. Example $1, \mathcal{H}$ can be thought of as representative of the Cartan symmetry class CI if the choice $\mathcal{T}_{\mu \nu}$ obeying $\mathcal{T}_{\mu \nu}^{2}=+\mathbb{I}$ and $\mathcal{C}_{\mu \nu}$ obeying $\mathcal{C}_{\mu \nu}^{2}=-\mathbb{I}$ for the symmetry under time-reversal and the spectral symmetry under charge-conjugation is dictated by a microscopic derivation of $\mathcal{H}$. Example $2, \mathcal{H}$ can be thought of as representative of the symmetry class DIII if the choice $\mathcal{T}_{\mu \nu}$ obeying 


\begin{tabular}{|l|l|l|l|l|}
\hline Triplet $(\mathscr{P}, \mathscr{T}, \mathscr{C})$ of symmetries in class CI & Generic perturbation $\mathcal{V}_{\mathscr{P} ; \mathscr{T} ; \mathscr{C}}$ & $\left(\eta_{\mathscr{T}}, \eta_{\mathscr{C}}\right)$ & Topological index & $\sigma\left(Q_{\mathscr{P} ; \mathscr{T} ; \mathscr{C} A}\right)$ \\
\hline \hline $\mathcal{P}_{01}, \mathcal{T}_{00}, \mathcal{C}_{12}$ & $v_{01} \mathcal{X}_{01}+v_{11} \mathcal{X}_{11}+v_{30} \mathcal{X}_{30}$ & $(+,-)$ & $\mathbb{Z}$ & $\circ$ \\
\hline $\mathcal{P}_{01}, \mathcal{T}_{11}, \mathcal{C}_{12}$ & $v_{01} \mathcal{X}_{01}+v_{11} \mathcal{X}_{11}+v_{21} \mathcal{X}_{21}$ & $(+,-)$ & $\mathbb{Z}$ & $\circ$ \\
\hline $\mathcal{P}_{01}, \mathcal{T}_{30}, \mathcal{C}_{12}$ & $v_{01} \mathcal{X}_{01}+v_{21} \mathcal{X}_{21}+v_{30} \mathcal{X}_{30}$ & $(+,-)$ & $\mathbb{Z}$ & $\circ$ \\
\hline $\mathcal{P}_{10}, \mathcal{T}_{00}, \mathcal{C}_{12}$ & $v_{01} \mathcal{X}_{01}+v_{03} \mathcal{X}_{03}+v_{11} \mathcal{X}_{11}+v_{13} \mathcal{X}_{13}$ & $(+,+)$ & 0 & \\
\hline $\mathcal{P}_{10}, \mathcal{T}_{11}, \mathcal{C}_{12}$ & $v_{01} \mathcal{X}_{01}+v_{02} \mathcal{X}_{02}+v_{11} \mathcal{X}_{11}+v_{12} \mathcal{X}_{12}$ & $(+,+)$ & 0 & $\times$ \\
\hline $\mathcal{P}_{10}, \mathcal{T}_{30}, \mathcal{C}_{12}$ & $v_{01} \mathcal{X}_{01}+v_{03} \mathcal{X}_{03}+v_{12} \mathcal{X}_{12}$ & $(-,+)$ & 0 & $\times$ \\
\hline $\mathcal{P}_{20}, \mathcal{T}_{00}, \mathcal{C}_{12}$ & $v_{01} \mathcal{X}_{01}+v_{03} \mathcal{X}_{03}+v_{22} \mathcal{X}_{22}$ & $(-,+)$ & 0 & $\times$ \\
\hline $\mathcal{P}_{20}, \mathcal{T}_{11}, \mathcal{C}_{12}$ & $v_{01} \mathcal{X}_{01}+v_{02} \mathcal{X}_{02}+v_{21} \mathcal{X}_{21}+v_{22} \mathcal{X}_{22}$ & $(+,+)$ & 0 & $\times$ \\
\hline $\mathcal{P}_{20}, \mathcal{T}_{30}, \mathcal{C}_{12}$ & $v_{01} \mathcal{X}_{01}+v_{03} \mathcal{X}_{03}+v_{21} \mathcal{X}_{21}+v_{23} \mathcal{X}_{23}$ & $(+,+)$ & 0 & $\times$ \\
\hline $\mathcal{P}_{31}, \mathcal{T}_{00}, \mathcal{C}_{12}$ & $v_{01} \mathcal{X}_{01}+v_{13} \mathcal{X}_{13}+v_{22} \mathcal{X}_{22}+v_{30} \mathcal{X}_{30}$ & $(+,+)$ & 0 & $\times$ \\
\hline $\mathcal{P}_{31}, \mathcal{T}_{11}, \mathcal{C}_{12}$ & $v_{01} \mathcal{X}_{01}+v_{12} \mathcal{X}_{12}+v_{22} \mathcal{X}_{22}$ & $(-,+)$ & 0 & $\times$ \\
\hline $\mathcal{P}_{31}, \mathcal{T}_{30}, \mathcal{C}_{12}$ & $v_{01} \mathcal{X}_{01}+v_{12} \mathcal{X}_{12}+v_{23} \mathcal{X}_{23}+v_{30} \mathcal{X}_{30}$ & $(+,+)$ & 0 & $\times$ \\
\hline
\end{tabular}

Table 2. The first column gives all possible combinations for the triplet of symmetries $\mathcal{P}_{\mu \nu}$, $\mathcal{T}_{\mu \nu}$, and $\mathcal{C}_{\mu \nu}$, from Table 1 that are compatible with the Cartan symmetry class CI defined by the conditions $\mathcal{T}_{\mu \nu}^{2}=+1$ and $\mathcal{C}_{\mu \nu}^{2}=-1$. The second column gives for each row the most general perturbation $\mathcal{V}_{\mathscr{P} ; \mathscr{T} ; \mathscr{C}}$ that obeys the triplet of symmetries $(\mathcal{P}, \mathcal{T}, \mathcal{C})$ on any given row. The third column gives the doublet $\left(\eta_{\mathscr{T}}, \eta_{\mathscr{C}}\right) \in\{-,+\} \times\{-,+\}$ where the sign $\eta_{\mathscr{T}}$ is defined by $\mathcal{P} \mathcal{T} \mathcal{P}=\eta_{\mathscr{T}} \mathcal{T}$ and similarly for $\eta_{\mathscr{C}}$. The fourth column is an application of the classification for the symmetry-protected topological band insulators in one-dimensional space derived in Refs. [15] and [16] (Table VI from Ref. [16] was particularly useful). The topological index $\mathbb{Z}$ and 0 correspond to topologically nontrivial and trivial bulk phases, respectively. The entry $\circ$ or $x$ in the last column denotes the presence or absence of zero modes in the spectrum $\sigma\left(\widetilde{Q}_{\mu \nu A}\right)$ as is explained in Sec. 4.4 and verified by numerics.

$\mathcal{T}_{\mu \nu}^{2}=-\mathbb{I}$ and $\mathcal{C}_{\mu \nu}$ obeying $\mathcal{C}_{\mu \nu}^{2}=+\mathbb{I}$ for the symmetry under time-reversal and the spectral symmetry under charge-conjugation is dictated by a microscopic derivation of $\mathcal{H}$. Example $3, \mathcal{H}$ can be thought of as representative of the Cartan symmetry class BDI if the choice $\mathcal{T}_{\mu \nu}$ obeying $\mathcal{T}_{\mu \nu}^{2}=+\mathbb{I}$ and $\mathcal{C}_{\mu \nu}$ obeying $\mathcal{C}_{\mu \nu}^{2}=+\mathbb{I}$ for the symmetry under time-reversal and the spectral symmetry under charge-conjugation is dictated by a microscopic derivation of $\mathcal{H}$. In fact, all complex Cartan symmetry classes AI, BDI, D, DIII, AII, CII, C, and CI are obtained from perturbing $\mathcal{H}$ under the condition that either a symmetry under time reversal or a spectral symmetry under charge conjugation is imposed by a microscopic derivation of $\mathcal{H}$. Finally, if a microscopic derivation of $\mathcal{H}$ does not prevent perturbations that break all the symmetries under time reversal and all the spectral symmetries under charge conjugations from Table 1 . then the remaining two real Cartan symmetry classes A and AIII are realized.

\subsection{Partition, topological numbers, and zero modes}

It is time to turn our attention to the topological properties of the single-particle Hamiltonian $\mathcal{H}$ defined by its matrix elements (4.1) or (4.2) in the orbital basis or in the Bloch basis, respectively.

The representation (4.2) demonstrates that the single-particle Hamiltonian $\mathcal{H}$ is reducible 
for all $k$ in the Brillouin zone,

$$
\begin{aligned}
& \mathcal{H}=\bigoplus_{n=1}^{N} \mathcal{H}_{\pi n / N}, \\
& \mathcal{H}_{k}=\mathcal{H}_{k}^{(+)} \oplus \mathcal{H}_{k}^{(-)},
\end{aligned}
$$

where the $4 \times 4$ Hermitian matrices $\mathcal{H}_{k}^{(+)}$and $\mathcal{H}_{k}^{(-)}$are isomorphic to the $2 \times 2$ Hermitian matrices

$$
H_{k}^{(+)}:=2 t \cos k \tau_{1}-2 \delta t \sin k \tau_{2}
$$

and

$$
H_{k}^{(-)}:=2 t \cos k \tau_{1}+2 \delta t \sin k \tau_{2}
$$

respectively.

The reducibility (4.14) defines the partition (2.9) for the one-dimensional example 4.2 . For simplicity, we take the thermodynamic limit $N \rightarrow \infty$ with $N=2 M$ so that we may define

$$
\mathfrak{H}_{A}:=\bigoplus_{n=1}^{M} \bigoplus_{\mu, \nu= \pm}\left|\chi_{\nu ; \pi n / 2 M}^{(\mu)}\right\rangle\left\langle\chi_{\nu ; \pi n / 2 M}^{(\mu)}\right|
$$

and

$$
\mathfrak{H}_{B}:=\bigoplus_{n=M+1}^{2 M} \bigoplus_{\mu, \nu= \pm}\left|\chi_{\nu ; \pi n / 2 M}^{(\mu)}\right\rangle\left\langle\chi_{\nu ; \pi n / 2 M}^{(\mu)}\right|
$$

Here, $\chi_{-; k}^{(+)}$and $\chi_{+; k}^{(+)}$are the pair of eigenstates with eigenvalues $\varepsilon_{-; k}^{(+)} \leqslant \varepsilon_{+; k}^{(+)}$of $H_{k}^{(+)}$. Similarly, $\chi_{-, k}^{(-)}, \chi_{+, k}^{(-)}$, and $\varepsilon_{-; k}^{(-)} \leqslant \varepsilon_{+; k}^{(-)}$denote the eigenstates and their eigenenergies from the lower and upper bands of $H_{k}^{(-)}$. This partition satisfies [recall Eq. 4.12] ]

$$
\begin{array}{ll}
\mathscr{P} A=B, & \mathscr{P} B=A, \\
\mathscr{C} A=A, & \mathscr{C} B=B, \\
\mathscr{T} A=A, & \mathscr{T} B=B, \\
\mathscr{S} A=A, & \mathscr{S} B=B .
\end{array}
$$

Hamiltonian (4.14c) describes a single-particle that hops between sites labeled by $\mathbf{\square}$ and $\square$ in Fig. 2 with the uniform hopping amplitude $t$ and the staggered hopping amplitude $\delta t$. Hamiltonian (4.14d) describes a single-particle that hops between sites labeled by $\bullet$ and $\circ$ in Fig. 2 with the uniform hopping amplitude $t$ and the staggered hopping amplitude $\delta t$.

If we take the thermodynamic limit

$$
N, N_{\mathrm{f}} \rightarrow \infty
$$

holding the fermion density

$$
N_{\mathrm{f}} / N=1
$$


fixed, we find the non-vanishing winding numbers

$$
W_{\mathrm{FS}}^{(+)}=-W_{\mathrm{FS}}^{(-)}:=\frac{\mathrm{i}}{2 \pi} \oint \mathrm{d} k \chi_{-}^{(+) \dagger}(k)\left(\frac{\partial \chi_{-}^{(+)}}{\partial k}\right)(k)
$$

for any non-vanishing dimerization $\delta$. Owing to the reducibility of $\mathcal{H}_{k}$, the winding number $W_{\mathrm{FS}}$ for the single-particle eigenstates making up the Fermi sea of $\mathcal{H}_{k}$ is

$$
W_{\mathrm{FS}}=W_{\mathrm{FS}}^{(+)}+W_{\mathrm{FS}}^{(-)}=0
$$

for any non-vanishing dimerization $\delta t$. The non-vanishing values of the winding numbers endow each of the single-particle Hamiltonians $H^{(+)}$and $H^{(-)}$with a topological attribute. The single-particle Hamiltonian $\mathcal{H}$ is topologically trivial as its winding number vanishes.

The very definition of the winding numbers (4.18) and (4.19) requires twisted boundary conditions and a spectral gap between the Fermi sea and all many-body excitations.

With open boundary conditions, two zero-dimensional boundaries at $r-1$ and $r$ follow from setting all hopping amplitudes between site $r-1$ and $r$ in Fig. 2 to zero, i.e., erasing the connecting full and dashed lines from Fig. 2 that intersect the vertical line $R V E$. In such an open geometry, the winding numbers (4.18) and (4.19) are ill defined. However, the bulk-edge correspondence implies the existence of mid-gap states (zero modes) that are localized at the boundaries (if the thermodynamic limit is taken with open boundary conditions), whenever the winding numbers (4.18) are non-vanishing (if the thermodynamic limit is taken with twisted boundary conditions). There are four such zero modes, a pair of zero modes for each boundary. On any given boundary, one of the zero modes localized on this boundary originates from $H_{r, r^{\prime}}^{(+)}$, while the other originates from $H_{r, r^{\prime}}^{(-)}$. These four zero modes are eigenstates of either $\mathcal{X}_{03}+\mathcal{X}_{33}$ or $\mathcal{X}_{03}-\mathcal{X}_{33}$. As a set, they are protected against any perturbation that anticommutes with $\mathcal{X}_{03}$ and $\mathcal{X}_{33}$, i.e., they are protected against any linear combination of $\mathcal{X}_{01}$, $\mathcal{X}_{02}, \mathcal{X}_{31}$, and $\mathcal{X}_{32}$. [We have verified by exact diagonalization that the zero modes in $\sigma(\mathcal{H})$ are indeed robust to the perturbations $\mathcal{X}_{01}, \mathcal{X}_{02}, \mathcal{X}_{31}$, or $\mathcal{X}_{32}$.]

It is believed that the existence of protected gapless boundary states, when taking the thermodynamic limit with open boundary conditions for a topological band insulator with Hamiltonian $\mathcal{H}$, implies the existence of protected gapless boundary states in the entanglement spectrum of $Q_{A}$ for a suitable partition of the form (2.9), when taking the thermodynamic limit with closed boundary conditions for the equal-time one-point correlation matrix $\mathcal{Q}$. [3, 12, 8, 13]

We have verified by exact diagonalization that this is also the case when we take the thermodynamic limit (4.17) of $\mathcal{H}$ defined by Eq. (4.14) with an even number of sites $N=2 M$ and with the partition defined by Eq. (4.15). The spectra for $\mathcal{H}$ and $Q_{A}$ are shown in Fig.3(a) for $\delta t=t$ and $N=12$. In both spectra, there are four zero modes within an exponential accuracy resulting from finite-size corrections. In the case of $\mathcal{H}$, a pair of zero modes is exponentially localized at one physical boundary a distance $2 M$ apart from the second pair of zero modes localized on the opposite boundary. In the case of $Q_{A}$, a pair of zero modes is exponentially localized at the boundary with $B$ to the left of $A$ a distance $M$ apart from the 

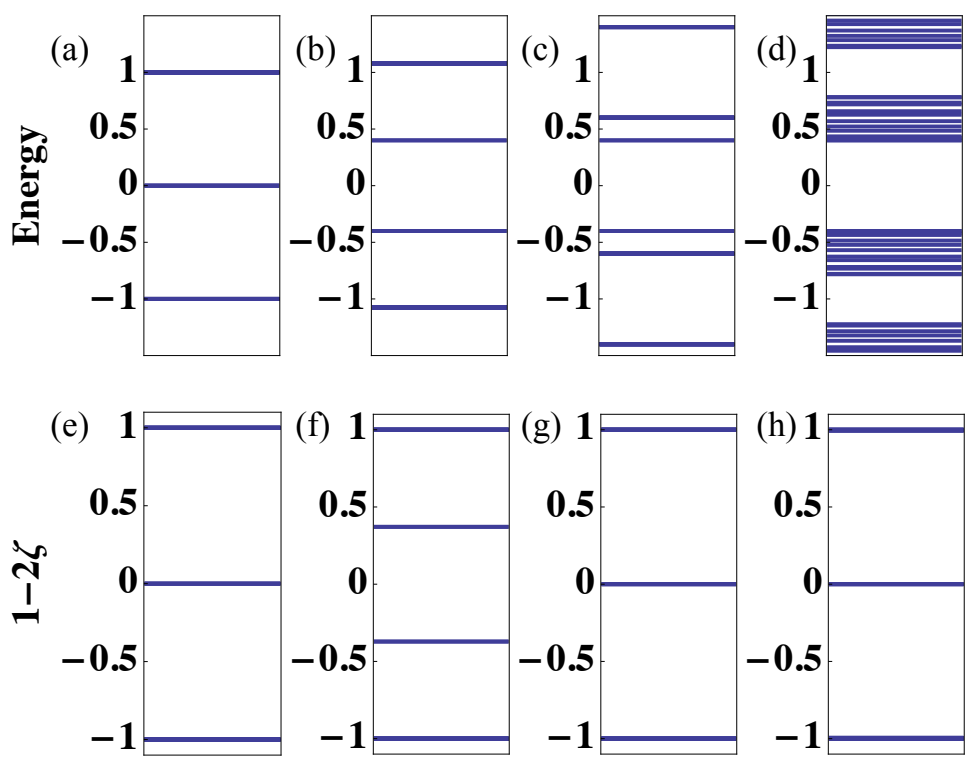

Figure 3. Energy spectra $\sigma\left(\widetilde{\mathcal{H}}_{\mu \nu}\right)$ in units of $2 t$ with $\widetilde{\mathcal{H}}_{\mu \nu}$ defined by Eq. 4.23 obeying open boundary conditions are plotted in panels (a-d). Entanglement spectra $\sigma\left(\widetilde{Q}_{\mu \nu}\right)$ for the equal-time one-point correlation matrix 2.8 derived from $\widetilde{\mathcal{H}}_{\mu \nu}$ obeying periodic boundary conditions are plotted in panels (f-h). The choices $\delta t=t$ and $\mathcal{V}_{\mu \nu}=0$ for panels (a) and (e), $\delta t=t$ and $\mathcal{V}_{12 r r^{\prime}}=\delta_{r, r^{\prime}}(t / 10) \mathcal{X}_{12}$ for panels (b) and (f), $\delta t=t$ and $\mathcal{V}_{11 r r^{\prime}}=\delta_{r, r^{\prime}}(t / 10) \mathcal{X}_{11}$ for panels (c) and $(\mathrm{g})$, and $\delta t=2 t / 3$ and $\mathcal{V}_{11 r r^{\prime}}=\delta_{r, r^{\prime}}(t / 10) \mathcal{X}_{11}$ for panels (d) and (h) are made. The spectra in panels (b) and (f) are unchanged if $\mathcal{V}_{12}$ is replaced by either $\mathcal{V}_{03}$ or $\mathcal{V}_{33}$.

second pair of zero modes exponentially localized at the boundary with $A$ to the left of $B$. The exponential decay of the zero modes away from their boundary is inversely proportional to the band gap of $\mathcal{H}$ when periodic boundary conditions are imposed.

We observe that the existence of zero modes in the spectrum of $Q_{A}$ does not imply the existence of zero modes in the spectrum of $\mathcal{H}$. For example, if we shift the spectrum of $\mathcal{H}$ defined in Eq. (4.14) in a uniform way by adding a chemical potential smaller than the band gap, we immediately lose the zero modes. However, all single-particle eigenstates are unperturbed by the chemical potential, for it enters as a perturbation that commutes with $\mathcal{H}$. Consequently, neither $\mathcal{Q}$, nor $Q_{A}$, nor their spectra depend on the chemical potential. In particular, the spectrum of $Q_{A}$ with a non-vanishing chemical potential contains the very same four zero modes that are present when the chemical potential vanishes.

The question we are after is the following. Are the four zero modes of $\mathcal{H}_{r, r^{\prime}}(t, \delta t)$, that are localized at both ends of an open chain, stable against perturbations that (i) commute with a reflection from Table 1 and (ii) have a characteristic energy that is small relative to the unperturbed band gap? Similarly, how stable are the four zero modes of $Q_{A}$ ? 


\subsection{Stability analysis of the zero modes}

The topological classification of non-interacting fermionic insulators based on the presence or absence of the discrete symmetries under the operations of time reversal, charge conjugation, and chirality satisfies a bulk-edge (holography) correspondence principle. According to this principle a non-vanishing value for a certain topological index defined for the bulk is equivalent to the existence of extended boundary states on physical boundaries. This correspondence is lost when crystalline symmetries such as inversion about a point or reflection about a mirror plane are also imposed. What remains is a symmetry-protected topological classification of non-interacting bulk fermionic insulators that obey a crystalline symmetry [15, 16]. This classification has two distinctive features. First, a symmetryprotected topologically phase is not required to support extended boundary states localized at the physical edges. Second, a bulk-edge correspondence principle is nevertheless believed to hold for the entanglement spectrum.

We are going to verify how this correspondence principle for symmetry-protected topologically phases of non-interacting fermionic insulating phases holds for the onedimensional model with the elementary building block defined by Eq. (4.1) and explain why. When $(\mu, \nu)=(0,0) ;(1,1) ;(2,1) ;(3,0)$, we are going to show that $\widetilde{\mathcal{H}}_{\mu \nu}$ defined in Eq. 4.23 fails to support robust edge states for an open geometry, whereas $\widetilde{\mathcal{Q}}_{\mu \nu A}$ supports robust edge states at the entangling boundaries.

To this end, our strategy is going to be to first explain the rows of Table 1. We shall then choose a combination of symmetry under time reversal and spectral symmetry under charge conjugation that puts $\widetilde{\mathcal{H}}_{\mu \nu}$ in the Cartan symmetry class CI. This symmetry class is a topologically trivial one, a generic perturbation of $\widetilde{\mathcal{H}}_{\mu \nu}$ destroys any boundary state that $\mathcal{H}_{\mu \nu}$ supports at a physical boundary. However, we shall show that imposing a suitable symmetry under parity guarantees the existence of protected boundary states in the entanglement spectrum $\sigma\left(\widetilde{\mathcal{Q}}_{\mu \nu A}\right)$.

If periodic boundary conditions are imposed, a perturbation that commutes with the parity transformation generated by $\mathcal{X}_{10}$ is of the general form

$$
\mathcal{V}_{k}:=\sum_{\nu=0}^{3}\left[\sum_{\mu=0,1} f_{\mu \nu ; k}+\sum_{\mu=2,3} g_{\mu \nu ; k}\right] \mathcal{X}_{\mu \nu}
$$

where the functions $f_{\mu \nu ; k}$ and $g_{\mu \nu ; k}$ are even and odd under the inversion $k \rightarrow-k$, respectively [recall Eq. $4.10 \mathrm{~b}$ ]. For simplicity, the perturbation $\mathcal{V}_{k}$ is taken independent of $k$, i.e.,

$$
\mathcal{V}_{k}:=\sum_{\nu=0}^{3} \sum_{\mu=0,1} v_{\mu \nu} \mathcal{X}_{\mu \nu}=\mathcal{X}_{10} V_{-k} \mathcal{X}_{10}
$$

with the eight parameters $v_{\mu \nu}$ real valued. In the orbital basis, we have

$$
\begin{aligned}
\mathcal{V}_{r, r^{\prime}}= & \delta_{r, r^{\prime}}\left[\underline{\underline{v_{01} \mathcal{X}_{01}}}+\underline{\underline{v_{02} \mathcal{X}_{02}}}+v_{00} \mathcal{X}_{00}+v_{03} \mathcal{X}_{03}\right] \\
& +\delta_{r, r^{\prime}}\left[\underline{v_{10} \mathcal{X}_{10}}+\underline{v_{11} \mathcal{X}_{11}}+\underline{v_{12} \mathcal{X}_{12}}+\underline{v_{13} \mathcal{X}_{13}}\right]
\end{aligned}
$$


The terms that have been underlined twice anti-commute with the two commuting matrices $\mathcal{X}_{03}$ and $\mathcal{X}_{33}$ that share the zero modes as eigenstates. Hence, the zero modes are protected as a set against the parity-preserving perturbations that are linear combinations of $\mathcal{X}_{01}$ and $\mathcal{X}_{02}$. All other terms in Eq. (4.22) fail to anti-commute with both $\mathcal{X}_{03}$ and $\mathcal{X}_{33}$. The zero modes are not necessarily protected as a set under these parity-preserving perturbations. The terms in Eq. (4.22) that are underlined once either anti-commute with $\mathcal{X}_{03}$ or $\mathcal{X}_{33}$ but not with both. The terms in Eq. 4.22) that are not underlined fail to anti-commute with both $\mathcal{X}_{03}$ and $\mathcal{X}_{33}$.

The same exercise can be repeated for the parity transformations generated by $\mathcal{X}_{01}, \mathcal{X}_{20}$, and $\mathcal{X}_{31}$. This deliver the first fourteen rows in Table 1 .

For any $\mu, \nu=0,1,2,3$, we define the single-particle Hamiltonian $\widetilde{\mathcal{H}}_{\mu \nu} \equiv \mathcal{H}+\mathcal{V}_{\mu \nu}$ by its matrix elements

$$
\begin{aligned}
& \widetilde{\mathcal{H}}_{\mu \nu r, r^{\prime}}:=\mathcal{H}_{r, r^{\prime}}+\mathcal{V}_{\mu \nu r, r^{\prime}} \\
& \mathcal{V}_{\mu \nu r, r^{\prime}}:=\delta_{r, r^{\prime}} v_{\mu \nu} \mathcal{X}_{\mu \nu} .
\end{aligned}
$$

The single-particle Hamiltonian $\mathcal{H}_{r, r^{\prime}}$ was defined in Eq. 4.1e for $r, r^{\prime}=1, \cdots, N-1$. We choose between imposing open boundary conditions by setting the hopping amplitudes to zero between sites $N$ and $N+1$ or periodic boundary conditions. The perturbation strength $v_{\mu \nu}$ is real-valued. It will be set to $t / 10$. The corresponding equal-time one-point correlation matrix defined by Eq. 2.8 is denoted $\widetilde{\mathcal{Q}}_{\mu \nu}$.

For illustrative purposes, we plot in Figs. 3 (a-d) the energy eigenvalue spectrum $\sigma\left(\widetilde{H}_{\mu \nu}\right)$ of $\widetilde{H}_{\mu \nu}$ obeying open boundary conditions by an exact diagonalization with $N=12$ that can be extrapolated to the thermodynamic limit. Energy eigenvalues are measured in units of $2 t$. In panels (a-c), $\delta t=t$ implies that all energy eigenstates have wave-functions that are localized on a pair of consecutive sites for which the hopping amplitude is $t+\delta t=2 t$. In the remaining panel (d), a $\delta t \neq t$ delocalizes bulk energy eigenstates that acquire a dispersion, i.e., a band width. The zero modes in panel (a) are four edge states. They are protected by the chiral symmetry in that they are eigenstates of either $\mathcal{X}_{03}$ or $\mathcal{X}_{33}$. For example, they are robust to changing the value of $\delta t$ away from $t$. However, these zero modes are shifted to non-vanishing energies by the perturbations $\mathcal{V}_{12}$ for panel (b) and $\mathcal{V}_{11}$ for panels (c-d). The spectrum in panel (b) is unchanged by the substitutions $\mathcal{V}_{12} \rightarrow \mathcal{V}_{03} \rightarrow \mathcal{V}_{33}$. The presence or absence of protected (against the perturbation from the second column) zero modes localized on the physical boundaries has been verified in this way for all rows and is reported in the penultimate column of Table 1

We plot in Figs. $3(\mathrm{e}-\mathrm{h})$ the eigenvalue spectrum $\sigma\left(\widetilde{Q}_{\mu \nu A}\right)$ of the upper-left block $\widetilde{Q}_{\mu \nu A}$ in the equal-time one-point correlation matrix $\widetilde{\mathcal{Q}}_{\mu \nu}$ corresponding to $\widetilde{\mathcal{H}}_{\mu \nu}$ obeying periodic boundary conditions by an exact diagonalization with $N=12$ that can be extrapolated to the thermodynamic limit. Panels (e-g) have $\delta t=t$. Panel (h) has $\delta t=2 t / 3$. There is no perturbation in panel (e), in which case four zero modes are present in the spectrum $\sigma\left(\widetilde{Q}_{\mu \nu A}\right)$. The perturbation $\mathcal{V}_{12}$ splits the four zero modes into two pairs of degenerate eigenstates with eigenvalues only differing by their sign in panel (f), as was the case for the Hamiltonian in panel (b). The spectrum in panel (f) is unchanged by the substitutions $\mathcal{V}_{12} \rightarrow \mathcal{V}_{03} \rightarrow \mathcal{V}_{33}$. 
Unlike in panels (c) and (d) for the Hamiltonian, panels (g) and (h) show that the four zero modes of $\widetilde{Q}_{\mu \nu A}$ are robust to the perturbation $\mathcal{V}_{11}$.

The lesson from Fig. 3 and Table 1 is that of all the seven

$$
\mathcal{V}_{00}, \mathcal{V}_{03}, \mathcal{V}_{11}, \mathcal{V}_{12}, \mathcal{V}_{21}, \mathcal{V}_{22}, \mathcal{V}_{30}
$$

out of fourteen parity-preserving perturbations that gap the zero modes of $\mathcal{H}$, only four, namely

$$
\mathcal{V}_{00}, \mathcal{V}_{11}, \mathcal{V}_{21}, \mathcal{V}_{30}
$$

fail to also gap the zero modes of $Q_{A}$.

To explain this observation, we rely on the reasoning that delivers Table 2 We assume that the underlying microscopic model has the triplet of symmetries $(\mathscr{P} ; \mathscr{T} ; \mathscr{C}) \sim$ $\left(\mathcal{P}_{\mu \nu}, \mathcal{T}_{\mu \nu}, \mathcal{C}_{\mu \nu}\right)$ where the doublet $(\mathscr{T}, \mathscr{C}) \sim\left(\mathcal{T}_{\mu \nu}, \mathcal{C}_{\mu \nu}\right)$ defines the symmetry class $\mathrm{CI}$, i.e., $\mathcal{T}_{\mu \nu}^{2}=+\mathbb{I}$ and $\mathcal{C}_{\mu \nu}^{2}=-\mathbb{I}$. We denote the most general perturbation that is compliant with the triplet of symmetries $(\mathscr{P} ; \mathscr{T} ; \mathscr{C})$ defining a given row of Table 2 by $\mathcal{V}_{\mathscr{P} ; \mathscr{T} ; \mathscr{C}}$. The explicit form of this perturbation is to be found in the second column of Table 2 as one varies $(\mathscr{P} ; \mathscr{T} ; \mathscr{C})$. For a given row in Table 2, $\mathcal{V}_{\mathscr{P} ; \mathscr{T} ; \mathscr{C}}$ is contained in the most general perturbation $\mathcal{V}_{\mathscr{T} ; \mathscr{C}}$ that is compliant with the doublet of symmetries $(\mathscr{T}, \mathscr{C})$. We define the single-particle Hamiltonian $\mathcal{H}_{\mathscr{P} ; \mathscr{T} ; \mathscr{C}} \equiv \mathcal{H}+\mathcal{V}_{\mathscr{P} ; \mathscr{T} ; \mathscr{C}}$ by its matrix elements

$$
\begin{aligned}
& \mathcal{H}_{\mathscr{P} ; \mathscr{T} ; \mathscr{C} r, r^{\prime}}:=\mathcal{H}_{r, r^{\prime}}+\mathcal{V}_{\mathscr{P} ; \mathscr{T} ; \mathscr{C} r, r^{\prime}} \\
& \mathcal{V}_{\mathscr{P} ; \mathscr{T} ; \mathscr{C} r, r^{\prime}}:=\delta_{r, r^{\prime}} \sum_{\mu, \nu \in \text { row }} v_{\mu \nu} \mathcal{X}_{\mu \nu}
\end{aligned}
$$

The corresponding equal-time one-point correlation matrix is $\mathcal{Q}_{\mathscr{P} ; \mathscr{T} ; \mathscr{C}}$ and its upper-left block is $Q_{\mathscr{P} ; \mathscr{T} ; \mathscr{C} A}$. The third column in Table 2 provides two signs for each row. The first sign $\eta_{\mathscr{T}}$ is positive if $\mathscr{P}$ commutes with $\mathscr{T}$ and negative if $\mathscr{P}$ anti-commutes with $\mathscr{T}$. The second sign $\eta_{\mathscr{C}}$ is positive if $\mathscr{P}$ commutes with $\mathscr{C}$ and negative if $\mathscr{P}$ anti-commutes with $\mathscr{C}$. The information contained with the doublet $\left(\eta_{\mathscr{T}}, \eta_{\mathscr{C}}\right)$ is needed to read from Table VI of Ref. [16] the bulk topological index of the single-particle Hamiltonian (4.26). This topological index does not guarantee that $\mathcal{H}_{\mathscr{P} ; \mathscr{T} ; \mathscr{C}}$ supports boundary states in an open geometry. In fact, $\mathcal{H}_{\mathscr{P} ; \mathscr{T} ; \mathscr{C}}$ does not support boundary states in an open geometry, for the physical boundaries are interchanged under the operation of parity $\mathscr{P}$. On the other hand, $Q_{\mathscr{P} ; \mathscr{T} ; \mathscr{C} A}$ supports boundary states on the entangling boundaries under the following conditions.

Hamiltonian $\mathcal{H}_{\mathscr{P} ; \mathscr{T} ; \mathscr{C}}$ is local by assumption and gaped if periodic boundary conditions are imposed. As explained in Sec. $3.2, \mathcal{Q}_{\mathscr{P} ; \mathscr{T} ; \mathscr{C}}$ and all its four blocks inherit this locality. We have shown with Eq. (2.38) that the upper-left block $Q_{\mathscr{P} ; \mathscr{T} ; \mathscr{C} A}$ inherits the symmetry $\mathcal{T}$ and the spectral symmetry $\mathcal{C}$ of $\mathcal{H}_{\mathscr{P} ; \mathscr{T} ; \mathscr{C}}$. We have also shown with Eq. (2.45) that the symmetry $\mathcal{P}$ of $\mathcal{H}_{\mathscr{P} ; \mathscr{T} ; \mathscr{C}}$ is turned into a spectral symmetry of $Q_{\mathscr{P} ; \mathscr{T} ; \mathscr{C} A}$ under $\Gamma_{\mathscr{P} A}:=C_{\mathscr{D} ; \mathscr{T} ; \mathscr{C} A B} P_{A B}^{\dagger}$. The unperturbed upper-left block $Q_{A}$ has two zero modes per entangling boundary. Because of locality, the perturbation

$$
\delta Q_{\mathscr{P} ; \mathscr{T} ; \mathscr{C} A}:=Q_{\mathscr{P} ; \mathscr{T} ; \mathscr{C} A}-Q_{A}
$$


only mixes the two members of a doublet of boundary states of $Q_{A}$ on a given entangling boundary. Hence, we may represent the effect of the perturbation $\delta Q_{\mathscr{P} ; \mathscr{T} ; \mathscr{C} A}$ by imposing on the Hermitian $2 \times 2$ matrix

$$
\delta Q_{\text {boundary }}:=\sum_{\mu=0}^{3} a_{\mu} \rho_{\mu}, \quad a_{\mu} \in \mathbb{R},
$$

( $\rho_{0}$ is the unit $2 \times 2$ matrix and $\rho_{1}, \rho_{2}, \rho_{3}$ are the Pauli matrices) the condition imposed by the symmetry $\mathscr{T} \sim \rho_{0} K$ and the spectral symmetries $\mathscr{C} \sim \rho_{2} K$ and $\Gamma_{\mathscr{P} A}$. The first two symmetries imply that

$$
\delta Q_{\text {boundary }}=a_{1} \rho_{1}+a_{3} \rho_{3} .
$$

A doublet of zero modes is thus protected if and only if

$$
\Gamma_{\mathscr{P} A}:=C_{\mathscr{P} ; \mathscr{T} ; \mathscr{C} A B} P_{A B}^{\dagger} \sim \rho_{0},
$$

for $\left\{\rho_{0}, \delta Q_{\text {boundary }}\right\}=0$ can then only be satisfied if $a_{1}=a_{3}=0$. We now show that condition 4.30 is only met for the first three rows of Table 2, the only rows from Table 2 with $\left(\eta_{\mathscr{T}}, \eta_{\mathscr{C}},\right)=(+,-)$, i.e., the only choice for the triplet $\left(\mathcal{P}_{\mu \nu}, \mathcal{T}_{\mu \nu}, \mathcal{C}_{\mu \nu}\right)$ for which

$$
[\mathscr{P}, \mathscr{T}]=0, \quad\{\mathscr{P}, \mathscr{C}\}=0
$$

To see this, we are going to combine Eq. (4.31) with

$$
\left[C_{\mathscr{P} ; \mathscr{T} ; \mathscr{C} A B}, T_{\mathscr{T}}\right]=0, \quad\left\{C_{\mathscr{P} ; \mathscr{T} ; \mathscr{C} A B}, C_{\mathscr{C}}\right\}=0
$$

where $T_{\mathscr{T}}$ and $C_{\mathscr{C}}$ represent the actions of time reversal and charge conjugation on the partition grading of the equal-time one-point correlation matrix. If we use the algebraic identity

$$
\begin{aligned}
{\left[\Gamma_{\mathscr{P} A}, T_{\mathscr{T}}\right] } & =C_{\mathscr{P} ; \mathscr{T} ; \mathscr{C} A B}\left[P_{A B}^{\dagger}, T_{\mathscr{T}}\right]+\left[C_{\mathscr{P} ; \mathscr{T} ; \mathscr{C} A B}, T_{\mathscr{T}}\right] P_{A B}^{\dagger}, \\
{\left[\Gamma_{\mathscr{P} A}, C_{\mathscr{C}}\right] } & =C_{\mathscr{P} ; \mathscr{T} ; \mathscr{C} A B}\left\{P_{A B}^{\dagger}, C_{\mathscr{C}}\right\}-\left\{C_{\mathscr{P} ; \mathscr{T} ; \mathscr{C} A B}, C_{\mathscr{C}}\right\} P_{A B}^{\dagger},
\end{aligned}
$$

Eq. (4.33), when combined with Eqs. (4.32) and (4.31), simplifies to

$$
\left[\Gamma_{\mathscr{P} A}, T_{\mathscr{T}}\right]=\left[\Gamma_{\mathscr{P} A}, C_{\mathscr{C}}\right]=0
$$

Equation (4.34) allows us to deduce that $\Gamma_{\mathscr{P} A}$ must be represented by $\rho_{0}$ on the twodimensional Hilbert space spanned by the boundary states on an entangling boundary,

$$
\Gamma_{\mathscr{P} A} \sim \rho_{0}
$$

Hence, the only $\delta Q_{\text {boundary }}$ in Eq. (4.29) that anti-commutes with $\rho_{0}$ is $\delta Q_{\text {boundary }}=0$, thereby proving the stability of the boundary states on an entangling boundary for the first three rows of Table 2 . 


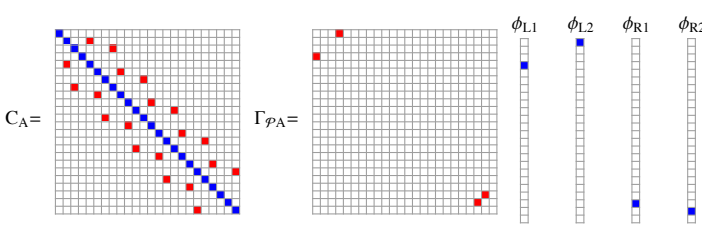

(a)

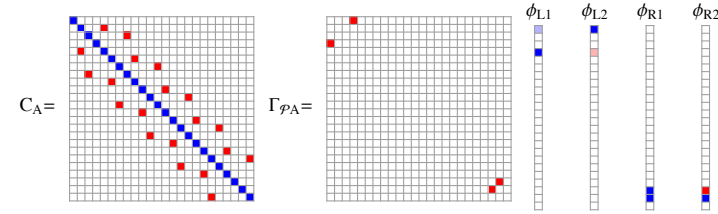

(b)

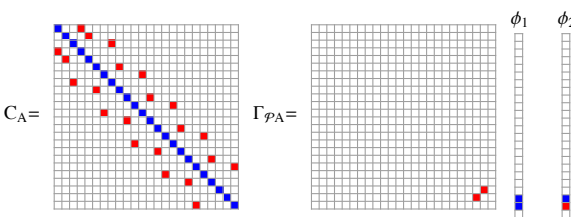

(c)

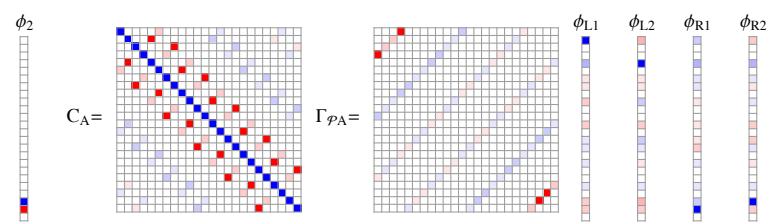

(d)

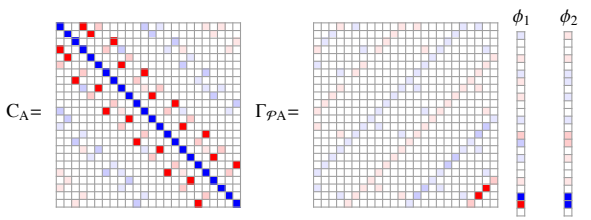

(e)

Figure 4. The one-dimensional lattice model is defined by Fig. 2 with $N:=N_{\text {tot }} / N_{\text {orb }}=$ $48 / 4=12$ repeat unit cells with either a torus or a cylindrical geometry. The partition is done by defining $A(B)$ to be the set of all the orbitals localized to the left (right) of the dashthree-dots (green) line $R V E$ in Fig. 2(a). The equal-time-correlation matrix $\mathcal{C}$ is defined in Eq. 2.5). It is a $48 \times 48$ Hermitian matrix with a $24 \times 24$ Hermitian block defining the matrix $C_{A}$, see Eq. 2.9c. The symmetry operation is the parity transformation (reflection) $\mathscr{P}$ about the dash-three-dots (green) line $R V E$ in Fig. 2(a). It interchanges $A$ and $B$ while leaving the entangling boundary $R V E$ unchanged. Hence, $\mathscr{P}$ can be represented by the $48 \times 48$ matrix $\mathcal{P}$ with the off-diagonal block structure displayed in Eq. 2.39b). There follows the existence of the $24 \times 24$ matrix $\Gamma_{\mathscr{P} A}$ defined in Eq. (2.45d) that anti-commutes with $Q_{A}:=\mathbb{I}-2 C_{A}$. The amplitudes of the matrix elements of $C_{A}$ and $\Gamma_{\mathscr{P} A}$ are represented by the coloring of the $24 \times 24$ elementary plaquettes of a square lattice. The blue (red) color of a plaquette determines the positive (negative) sign of the matrix element. The lighter the color, the smaller the magnitude with white representing zero. The darker the color, the larger the magnitude with 0.5 the largest magnitude. A $24 \times 1$ rectangular lattice represents as a column vector an eigenstate of $Q_{A}:=\mathbb{I}-2 C_{A}$. (a) The case of Hamiltonian 4.1c) obeying periodic boundary conditions for $\delta t=t$. The four entangling zero modes are also plotted. (b) The case of Hamiltonian 4.1c) obeying periodic boundary conditions for $\delta t=t$ and perturbed by $0.05 t \mathcal{X}_{11}$. The four entangling zero modes are also plotted. (c) The case of Hamiltonian 4.1c obeying open boundary conditions for $\delta t=t$ and perturbed by $0.05 t \mathcal{X}_{11}$. The two entangling zero modes are also plotted. (d) The case of Hamiltonian 4.1c obeying periodic boundary conditions for $\delta t=9 t / 11$ and perturbed by $0.05 t \mathcal{X}_{11}$. The four entangling zero modes are also plotted. (e) The case of Hamiltonian (4.1c) obeying open boundary conditions for $\delta t=9 t / 11$ and perturbed by $0.05 t \mathcal{X}_{11}$. The two entangling zero modes are also plotted.

\subsection{Numerical verification that $\Gamma_{\mathscr{P}}$ is local}

For completeness, we verify numerically the prediction from $S e c .3 .2$ that $\Gamma_{\mathscr{P} A}$ is local in that it does not mix zero modes localized on boundaries separated by a bulk-like distance. To 
this end, we consider Hamiltonian (4.1c) with or without perturbations for $N_{\text {tot }}=48$ orbitals (twelve repeat unit cells with four orbitals per repeat unit cell, $N=12$ and $N_{\text {orb }}=4$ ). Plotted in Fig. 4 as columnar vectors in the orbital basis are the zero modes $\phi$ that are localized at the entangling boundaries.

When periodic boundary conditions are imposed, there are two entangling boundaries separated by the bulk-like distance of order $N / 2=6$. Correspondingly, there are two zero modes $\phi_{\mathrm{L} 1}$ and $\phi_{\mathrm{L} 2}$ localized on the left entangling boundary and there are two zero modes $\phi_{\mathrm{R} 1}$ and $\phi_{\mathrm{R} 2}$ localized on the right entangling boundary. They are represented in Figs. 4(a), 4(b), and 4(d) as columnar vectors in the orbital basis. One verifies that the overlap of any pair of zero modes with one zero mode localized on the left entangling boundary and the other zero mode localized on the right entangling boundary are exponentially suppressed in magnitude by a factor of order $\exp (-b r \Delta)$ with $b$ a number or order unity and the separation $r$ of order $N / 2=6$.

When open boundary conditions are imposed, there are two physical boundaries separated by the bulk-like distance of order $N / 2=6$. and one entangling boundary a distance of order $N / 4=3$ away from either physical boundaries. The perturbation $0.05 t \mathcal{X}_{11}$ has been added to the Hamiltonian (4.1c). According to Table 1, this perturbation gaps the zero modes localized on the physical boundaries. Correspondingly, there are two zero modes $\phi_{1}$ and $\phi_{2}$ localized on the entangling boundary represented in Figs. 4 (c) and 4 (e) by columnar vectors in the orbital basis.

\section{Topological insulator protected by one reflection symmetry in two dimensions}

\subsection{Hamiltonian and topological quantum numbers}

Our second example is defined by choosing $d=2$ and $N_{\text {orb }}=4$ in Eq. 2.1. We represent the action on the orbital degrees of freedom by the $4 \times 4$ matrices defined in Eq. (4.9a). The Brillouin zone (BZ) is two-dimensional and the single-particle Hamiltonian admits the direct-sum decomposition [14]

$$
\begin{aligned}
& \mathcal{H}=\bigoplus_{\boldsymbol{k} \in \mathrm{BZ}} \mathcal{H}_{\boldsymbol{k}}, \\
& \mathcal{H}_{\boldsymbol{k}}:=\left[2 t\left(\cos k_{1}+\cos k_{2}\right)-\mu\right] \mathcal{X}_{03}-2 \Delta \sin k_{1} \mathcal{X}_{31}-2 \Delta \sin k_{2} \mathcal{X}_{02},
\end{aligned}
$$

with the real-valued characteristic energy scales $t, \Delta$, and $\mu$.

Hamiltonian $\mathcal{H}$ can be interpreted as the direct sum

$$
\mathcal{H}_{k}=\mathcal{H}_{k}^{(+)} \oplus \mathcal{H}_{k}^{(-)}
$$

of the Bogoliubov-de-Gennes Hamiltonian

$$
\mathcal{H}_{k}^{(+)} \sim\left[2 t\left(\cos k_{1}+\cos k_{2}\right)-\mu\right] \tau_{3}-2 \Delta\left[\sin k_{1} \tau_{1}+\sin k_{2} \tau_{2}\right]
$$

describing $p_{1}+\mathrm{i} p_{2}$ superconducting order and the Bogoliubov-de-Gennes Hamiltonian

$$
\mathcal{H}_{k}^{(-)} \sim\left[2 t\left(\cos k_{1}+\cos k_{2}\right)-\mu\right] \tau_{3}+2 \Delta\left[\sin k_{1} \tau_{1}-\sin k_{2} \tau_{2}\right]
$$


describing $p_{1}-\mathrm{i} p_{2}$ superconducting order. A gap is present when $|\mu|<4 t$, an assumption that is made throughout in Sec. 5 .

The bundle of single-particle eigenstates obtained by collecting the lower band from the bundle $\left\{\mathcal{H}_{\boldsymbol{k}}^{(+)}, \boldsymbol{k} \in \mathrm{BZ}\right\}$ has the opposite non-vanishing Chern number to that of the bundle of single-particle eigenstates obtained by collecting the lower band from the bundle $\left\{\mathcal{H}_{\boldsymbol{k}}^{(-)}, \boldsymbol{k} \in \mathrm{BZ}\right\}$. The bundle of single-particle eigenstates obtained by collecting the lower band from the bundle $\left\{\mathcal{H}_{k}, k \in \mathrm{BZ}\right\}$ is topologically trivial.

\subsection{Symmetries}

The symmetries of the single-particle Hamiltonian defined by Eq. (5.1) are the following. [1] There are two symmetries of the inversion type. If $\mathscr{I}$ denotes the inversion of space

$$
\mathscr{I}: \boldsymbol{r} \mapsto-\boldsymbol{r}
$$

then

$$
\mathcal{O}_{\mathscr{I}}^{\dagger} \mathcal{H}_{-k} \mathcal{O}_{\mathscr{I}}=\mathcal{H}_{+k}
$$

with

$$
\mathcal{O}_{\mathscr{I}} \in\left\{\mathcal{X}_{03}, \mathcal{X}_{33}\right\}
$$

[2] There are two symmetries of the reflection about the horizontal axis $\boldsymbol{k}=\left(k_{1}, 0\right)$ type. If $\mathscr{R}_{1}$ denotes the reflection

$$
\mathscr{R}_{1}: r_{1} \mapsto+r_{1}, \quad \mathscr{R}_{1}: r_{2} \mapsto-r_{2},
$$

then

$$
\mathcal{O}_{\mathscr{R}_{1}}^{\dagger} \mathcal{H}_{+k_{1},-k_{2}} \mathcal{O}_{\mathscr{R}_{1}}=\mathcal{H}_{+\boldsymbol{k}}
$$

with

$$
\mathcal{O}_{\mathscr{R}_{1}} \in\left\{\mathcal{X}_{13}, \mathcal{X}_{23}\right\}
$$

[3] There are two symmetries of the reflection about the vertical axis $\boldsymbol{k}=\left(0, k_{2}\right)$ type. If $\mathscr{R}_{2}$ denotes the reflection

$$
\mathscr{R}_{2}: r_{1} \mapsto-r_{1}, \quad \mathscr{R}_{2}: r_{2} \mapsto+r_{2}
$$

then

$$
\mathcal{O}_{\mathscr{R}_{2}}^{\dagger} \mathcal{H}_{-k_{1},+k_{2}} \mathcal{O}_{\mathscr{R}_{2}}=\mathcal{H}_{+k}
$$

with

$$
\mathcal{O}_{\mathscr{R}_{2}} \in\left\{\mathcal{X}_{10}, \mathcal{X}_{20}\right\}
$$

[4] There are two symmetries of the time-reversal type. If $\mathscr{T}$ denotes the reversal of time

$$
\mathscr{T}: t \mapsto-t
$$


(a)

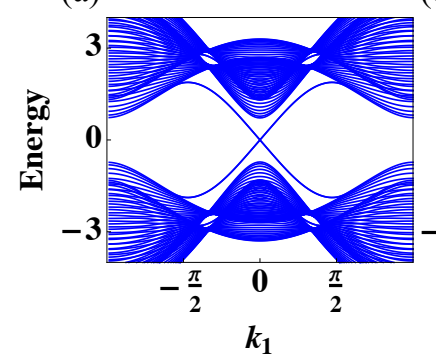

(e)

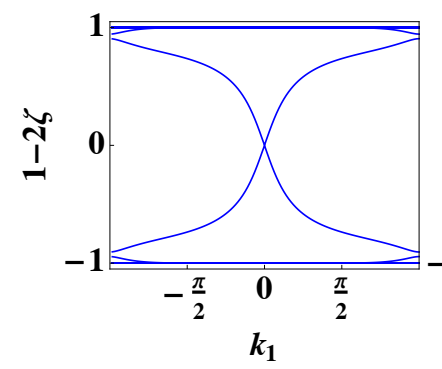

(b)

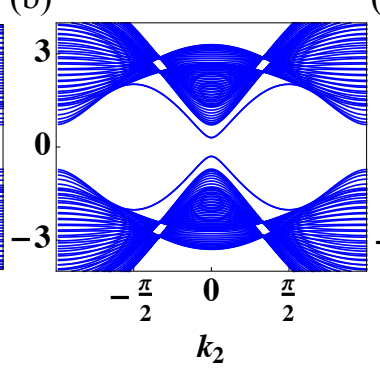

(f) (c)

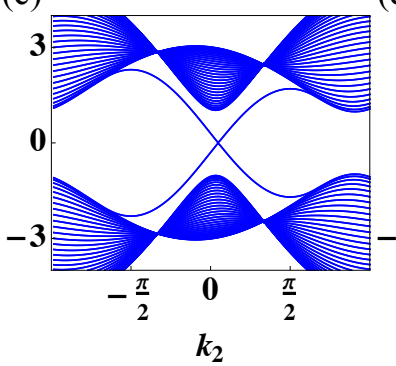

(d)

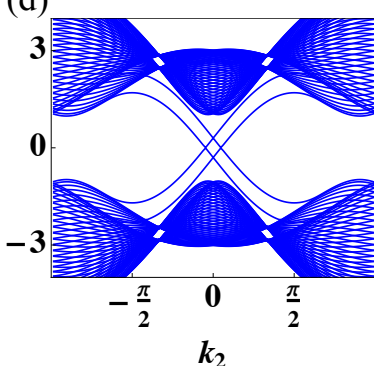

(h)
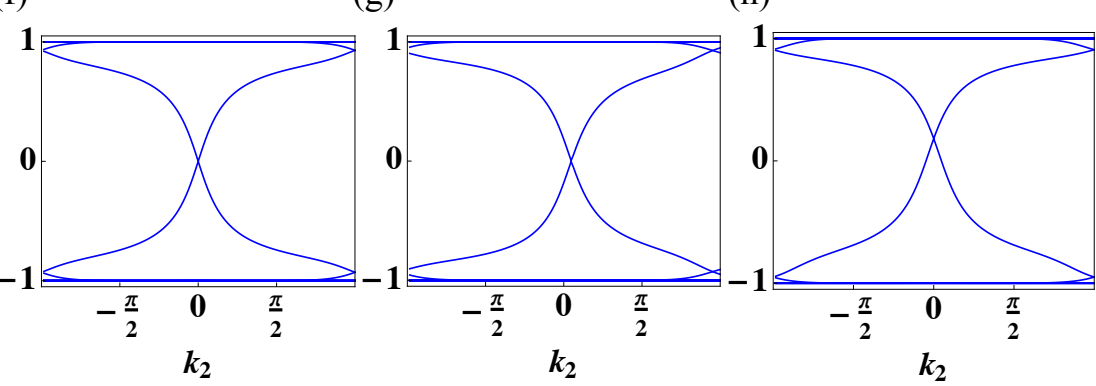

Figure 5. (Color online) (a-d) Single-particle energy spectra of $\widetilde{\mathcal{H}}_{k_{i} \mu \nu}$ defined in Eq. 5.15 with $i=1$, 2. (e-f) Single-particle spectra $1-2 \zeta$ of $\widetilde{\mathcal{Q}}_{k_{i} \mu \nu A_{i+1}}$ with $i+1$ defined modulo 2 . The energy scales in $\widetilde{\mathcal{H}}_{k_{i} \mu \nu}$ are chosen to be $t=\Delta=-\mu=1$, see Eq. $5.1 \mathrm{~b}$. The choice of $\mu=0,1,2,3$ and $\nu=0,1,2,3$ determines the perturbation to Eq. 5.1b. It is $0.3 t \mathcal{X}_{13}$ for panels $(\mathrm{a}, \mathrm{b}, \mathrm{e}, \mathrm{f})$. It is $0.3 t \mathcal{X}_{02}$ for panels $(\mathrm{c}, \mathrm{g})$. It is $0.3 t \mathcal{X}_{32}$ for panels $(\mathrm{d}, \mathrm{h})$.

then

$$
\mathcal{O}_{\mathscr{T}}^{\dagger} \mathcal{H}_{-k}^{*} \mathcal{O}_{\mathscr{T}}=\mathcal{H}_{+k}
$$

with

$$
\mathcal{O}_{\mathscr{T}} \in\left\{\mathcal{X}_{10}, \mathcal{X}_{20}\right\}
$$

[5] There are two spectral symmetries of the charge-conjugation type,

$$
\mathcal{O}_{\mathscr{C}}^{\dagger} \mathcal{H}_{-\boldsymbol{k}}^{\mathrm{T}} \mathcal{O}_{\mathscr{C}}=-\mathcal{H}_{+\boldsymbol{k}}
$$

with

$$
\mathcal{O}_{\mathscr{C}} \in\left\{\mathcal{X}_{01}, \mathcal{X}_{31}\right\}
$$

[6] There are two spectral symmetries of the chiral type,

$$
\mathcal{O}_{\mathscr{S}}^{\dagger} \mathcal{H}_{k} \mathcal{O}_{\mathscr{S}}=-\mathcal{H}_{k}
$$

with

$$
\mathcal{O}_{\mathscr{C}} \in\left\{\mathcal{X}_{11}, \mathcal{X}_{21}\right\}
$$

\subsection{Partition and zero modes}

From now on, we adopt a cylindrical geometry instead of the torus geometry from Sec. 5.1, i.e., we compactify two-dimensional space $\left\{\boldsymbol{x}:=\left(x_{1}, x_{2}\right) \in \mathbb{R}^{2}\right\}$ only along one 
Cartesian coordinate. Consequently, only one component of the two-dimensional momentum $\left\{\boldsymbol{k}:=\left(k_{1}, k_{2}\right) \in \mathbb{R}^{2}\right\}$ is chosen to be a good quantum number in Eq. (5.1).

For any choice of the direction $i=1,2$ along which periodic boundary conditions are imposed while open boundary conditions are imposed along the orthogonal direction $i+1$ modulo 2 , for any positive integer $M_{i}$, and for any good momentum quantum number

$$
k_{i}=\frac{2 \pi}{2 M_{i} \mathfrak{a}} n_{i}, \quad n_{i}=1, \cdots, 2 M_{i}, \quad i=1,2,
$$

we do the following. First, we denote by $\mathcal{H}_{k_{i}}$ the $8 M_{i+1} \times 8 M_{i+1}$ Hermitian matrix such that the one-dimensional Fourier transform of

$$
\mathcal{H}_{k_{i} r_{i+1}, r_{i+1}^{\prime}}:=\left\langle r_{i+1}\left|\mathcal{H}_{k_{i}}\right| r_{i+1}^{\prime}\right\rangle
$$

delivers Eq. (5.1b). Here,

$$
r_{i+1}=n_{i+1} \mathfrak{a}, \quad n_{i+1}=1, \cdots, 2 M_{i+1},
$$

and

$$
r_{i+1}^{\prime}=n_{i+1}^{\prime} \mathfrak{a}, \quad n_{i+1}^{\prime}=1, \cdots, 2 M_{i+1},
$$

are the lattice sites from an open chain along the direction $i+1$ (defined modulo 2) of a rectangular lattice with the lattice spacing $\mathfrak{a}$ and the number of lattice sites $M_{i+1}$ along the direction $i+1$ modulo 2. Second, we define the $k_{i}$-dependent partition

$$
\mathfrak{H}:=\bigoplus_{k_{i}} \mathfrak{H}_{k_{i}}, \mathfrak{H}_{k_{i}}:=\mathfrak{H}_{A_{i+1}} \oplus \mathfrak{H}_{B_{i+1}}
$$

where

$$
\begin{aligned}
\mathfrak{H}_{A_{i+1}} & :=\bigoplus_{n_{i+1}=1}^{M_{i+1}} \bigoplus_{\alpha=1}^{4}\left|k_{i}, n_{i+1}, \alpha\right\rangle\left\langle k_{i}, n_{i+1}, \alpha\right|, \\
\mathfrak{H}_{B_{i+1}}:= & \bigoplus_{n_{i+1}}^{2 M_{i+1}} \bigoplus_{i+1}^{4}\left|k_{i}, n_{i+1}, \alpha\right\rangle\left\langle k_{i}, n_{i+1}, \alpha\right|,
\end{aligned}
$$

and the ket $\left|k_{i}, n_{i+1}, \alpha\right\rangle$ denotes the single-particle state with the Bloch index $k_{i}$, for it is extended along the $i=1,2$ direction, the unit repeat cell index $n_{i+1}$, for it is localized at the site $n_{i+1} \mathfrak{a}$ along the $i+1$ modulo 2 direction, and the orbital index $\alpha=1,2,3,4$. If we denote by $\mathscr{R}_{i}$ the reflection that leaves $k_{i}$ unchanged but reverses the sign of $k_{i+1}$, i.e.,

$$
\mathscr{R}_{i} k_{i}=+k_{i}, \quad \mathscr{R}_{i} k_{i+1}=-k_{i+1},
$$

we then have that

$$
\begin{array}{ll}
\mathscr{R}_{i} A_{i+1}=B_{i+1}, & \mathscr{R}_{i} B_{i+1}=A_{i+1}, \\
\mathscr{C} A_{i+1}=A_{i+1}, & \mathscr{C} B_{i+1}=B_{i+1}, \\
\mathscr{T} A_{i+1}=A_{i+1}, & \mathscr{T} B_{i+1}=B_{i+1}, \\
\mathscr{S} A_{i+1}=A_{i+1}, & \mathscr{S} B_{i+1}=B_{i+1} .
\end{array}
$$


The thermodynamic limit is defined by

$$
N, N_{\mathrm{f}} \rightarrow \infty,
$$

with $N=\left(2 M_{1}\right) \times\left(2 M_{2}\right)$, holding the fermion density

$$
N_{\mathrm{f}} / N=1
$$

fixed. In the thermodynamic limit, the single-particle energy eigenvalue spectrum $\sigma\left(\mathcal{H}_{i}\right)$ of

$$
\mathcal{H}_{i}:=\bigoplus_{k_{i}} \mathcal{H}_{k_{i}}
$$

supports two pairs of zero modes, each of which are localized at the opposite (physical) boundaries on the cylinder whose symmetry axis coincides with the direction $i+1$ (defined modulo 2) along which open boundary conditions have been imposed. Similarly, the entanglement spectrum $\sigma\left(Q_{A_{i+1}}\right)$ of the equal-time one-point correlation matrix

$$
Q_{A_{i+1}}:=\mathbb{I}-2 C_{A_{i+1}}
$$

supports a pair of zero modes localized at the entangling boundary.

In order not to confuse gapless boundary modes originating from the physical boundaries with gapless boundary modes originating from the entangling boundary, whenever the physical boundaries support gapless boundary states in the energy spectrum, we opt for a torus geometry when computing the entanglement spectrum.

Desired is a study of the stability of these zero modes under all generic perturbations of $\mathcal{H}_{i}$ that respect any one $(\mathscr{S})$ of the two chiral symmetry from Eq. $(5.8)$ and any one $(\mathscr{R})$ of the four reflection symmetries from Eqs. (5.4) and (5.5).

\subsection{Stability analysis of the zero modes}

5.4.1. Definitions of $\widetilde{\mathcal{H}}_{i \mu \nu}$ and $\widetilde{\mathcal{Q}}_{k_{i} \mu \nu A_{i+1}}$ To begin with the stability analysis, we choose $i=1,2$ and perturb Hamiltonian $5.9 \mathrm{~b}$ by adding locally any one of the sixteen matrices $\mathcal{X}_{\mu \nu} \equiv \sigma_{\mu} \otimes \tau_{\nu}$ parametrized by $\mu, \nu=0,1,2,3$. Thus, we define

$$
\widetilde{\mathcal{H}}_{i \mu \nu}:=\bigoplus_{k_{i}} \widetilde{\mathcal{H}}_{k_{i} \mu \nu} \equiv \bigoplus_{k_{i}}\left(\mathcal{H}_{k_{i}}+\mathcal{V}_{\mu \nu}\right)
$$

by the matrix elements

$$
\begin{aligned}
& \widetilde{\mathcal{H}}_{k_{i} \mu \nu r_{i+1}, r_{i+1}^{\prime}}:=\mathcal{H}_{k_{i} r_{i+1}, r_{i+1}^{\prime}}+\mathcal{V}_{\mu \nu r_{i+1}, r_{i+1}^{\prime}}, \\
& \mathcal{V}_{\mu \nu r_{i+1}, r_{i+1}^{\prime}}:=\delta_{r_{i+1}, r_{i+1}^{\prime}} v_{\mu \nu} \mathcal{X}_{\mu \nu},
\end{aligned}
$$

where the matrix elements of $\mathcal{H}_{k_{i}}$ were defined in Eq. (5.9) and the perturbation strength $v_{\mu \nu}$ is real valued. Equipped with $\widetilde{\mathcal{H}}_{k_{i} \mu \nu}$, we define $\widetilde{\mathcal{Q}}_{k_{i} \mu \nu A_{i+1}}$ from Eq. 2.11 with the caveat that periodic boundary conditions are imposed along the direction $i+1$ (modulo 2). 


\begin{tabular}{|c|c|c|c|c|c|c|c|c|c|c|c|c|c|c|}
\hline \multirow{2}{*}{$\mathcal{X}_{\mu \nu} \equiv \sigma_{\mu} \otimes \tau_{\nu}$} & \multicolumn{2}{|c|}{$\mathscr{R}_{1}$} & \multicolumn{2}{|c|}{$\mathscr{R}_{2}$} & \multicolumn{2}{|c|}{$\mathscr{T}$} & \multicolumn{2}{|c|}{$\mathscr{C}$} & \multicolumn{2}{|c|}{$\mathscr{S}$} & \multirow{2}{*}{\multicolumn{2}{|c|}{$\sigma\left(\widetilde{\mathcal{H}}_{k_{1} \mu \nu}\right) \quad \sigma\left(\widetilde{Q}_{k_{1} \mu \nu A_{2}}\right)$}} & \multirow{2}{*}{$\sigma\left(\widetilde{\mathcal{H}}_{k_{2} \mu \nu}\right)$} & \multirow{2}{*}{$\sigma\left(\widetilde{Q}_{k_{2} \mu \nu A_{1}}\right)$} \\
\hline & $\mathcal{R}_{13}$ & $\mathcal{R}_{23}$ & $\mathcal{R}_{10}$ & $\mathcal{R}_{20}$ & $\mathcal{T}_{10}$ & $\mathcal{T}_{20}$ & $\mathcal{C}_{01}$ & $\mathcal{C}_{31}$ & $\mathcal{S}_{11}$ & $\mathcal{S}_{21}$ & & & & \\
\hline $\mathcal{X}_{00}$ & 0 & $\circ$ & $\circ$ & $\circ$ & 0 & $\circ$ & $\times$ & $\times$ & $\times$ & $\times$ & $\otimes$ & $\circ$ & $\otimes$ & $\circ$ \\
\hline $\mathcal{X}_{01}$ & $\times$ & $\times$ & 0 & 0 & 0 & 0 & $\times$ & $\times$ & $\times$ & $\times$ & $\otimes$ & $\otimes$ & 0 & $\circ$ \\
\hline $\mathcal{X}_{02}$ & $\times$ & $x$ & 0 & 0 & $x$ & $x$ & $\times$ & $x$ & 0 & 0 & 0 & 0 & $\otimes$ & $\otimes$ \\
\hline $\mathcal{X}_{03}$ & 0 & 0 & 0 & 0 & 0 & 0 & 0 & 0 & 0 & 0 & 0 & $\circ$ & 0 & 0 \\
\hline $\mathcal{X}_{10}$ & 0 & $\times$ & $\circ$ & $\times$ & 0 & $\times$ & $\times$ & $\circ$ & $x$ & $\circ$ & $x$ & $\circ$ & 0 & $\circ$ \\
\hline $\mathcal{X}_{11}$ & $\times$ & 0 & $\circ$ & $x$ & 0 & $\times$ & $x$ & 0 & $x$ & 0 & $\times$ & $\times$ & $\times$ & $\times$ \\
\hline $\mathcal{X}_{12}$ & $\times$ & 0 & $\circ$ & $x$ & $x$ & $\circ$ & $\times$ & 0 & $\circ$ & $x$ & 0 & $\circ$ & 0 & $\circ$ \\
\hline $\mathcal{X}_{13}$ & $\circ$ & $x$ & 0 & $\times$ & 0 & $\times$ & $\circ$ & $x$ & $\circ$ & $x$ & $\circ$ & $\circ$ & $x$ & o \\
\hline $\mathcal{X}_{20}$ & $x$ & $\circ$ & $x$ & 0 & 0 & $x$ & $\circ$ & $\times$ & $\circ$ & $x$ & $x$ & $\circ$ & 0 & $\circ$ \\
\hline $\mathcal{X}_{21}$ & $\circ$ & $\times$ & $\times$ & 0 & 0 & $\times$ & $\circ$ & $\times$ & $\circ$ & $\times$ & $\times$ & $\times$ & $\times$ & $\times$ \\
\hline $\mathcal{X}_{22}$ & 0 & $x$ & $x$ & 0 & $\times$ & $\circ$ & $\circ$ & $\times$ & $x$ & 0 & 0 & 0 & 0 & $\circ$ \\
\hline $\mathcal{X}_{23}$ & $\times$ & $\circ$ & $x$ & 0 & 0 & $\times$ & $x$ & $\circ$ & $x$ & 0 & $\circ$ & $\circ$ & $\times$ & $\circ$ \\
\hline $\mathcal{X}_{30}$ & $x$ & $\times$ & $\times$ & $\times$ & $x$ & $x$ & $\times$ & $\times$ & $\circ$ & $\circ$ & $\otimes$ & 0 & $\otimes$ & $\circ$ \\
\hline $\mathcal{X}_{31}$ & 0 & $\circ$ & $x$ & $\times$ & $x$ & $x$ & $x$ & $x$ & 0 & 0 & $\otimes$ & $\otimes$ & 0 & $\circ$ \\
\hline $\mathcal{X}_{32}$ & 0 & 0 & $\times$ & $\times$ & 0 & $\circ$ & $\times$ & $\times$ & $\times$ & $x$ & 0 & 0 & $\otimes$ & $\otimes$ \\
\hline $\mathcal{X}_{33}$ & $x$ & $x$ & $x$ & $x$ & $x$ & $x$ & 0 & 0 & $x$ & $x$ & $\circ$ & $\circ$ & 0 & $\circ$ \\
\hline
\end{tabular}

Table 3. The spectrum $\sigma\left(\widetilde{\mathcal{H}}_{k_{i} \mu \nu}\right)$ of the single-particle Hamiltonian $\widetilde{\mathcal{H}}_{k_{i} \mu \nu}$ defined by Eq. 5.15 and obeying periodic boundary conditions along the $i=1,2$ direction and open boundary conditions along the $i+1$ (modulo 2) direction. The entanglement spectrum $\sigma\left(\widetilde{Q}_{k_{i} \mu \nu A_{i+1}}\right)$ defined by Eq. 2.11 for the single-particle Hamiltonian $\widetilde{\mathcal{H}}_{k_{i} \mu \nu}$ obeying periodic boundary conditions along both the $i=1,2$ and the $i+1$ (modulo 2) direction. The entry $\circ$ or $\times$ in the second to sixth columns denotes the presence or the absence, respectively, of the symmetries under reflections about the directions $1\left(\mathscr{R}_{1}\right)$ and $2\left(\mathscr{R}_{2}\right)$, charge conjugation $\mathscr{C}$, time reversal $\mathscr{T}$, and chiral $\mathscr{S}$ of the perturbation $\delta_{r_{i+1}, r_{i+1}^{\prime}} 0.3 t \mathcal{X}_{\mu \nu}$ for the sixteen rows. In the last two columns, the entries $\circ$ and $\times$ denote the presence and the absence, respectively, of non-propagating zero modes in the spectra $\sigma\left(\widetilde{\mathcal{H}}_{k_{i} \mu \nu}\right)$ and $\sigma\left(\widetilde{\mathcal{Q}}_{k_{i} \mu \nu A_{i+1}}\right)$ as determined by extrapolation to the thermodynamic limit of exact diagonalization with the open direction running over 32 repeat unit cells and the momentum along the compactified direction running over 128 values. The entry $\otimes$ in the last two columns denotes the existence of crossings between the mid-gap branches, whereby the crossings are away from vanishing energy (entanglement eigenvalue) and vanishing momentum in the spectra $\sigma\left(\widetilde{\mathcal{H}}_{k_{i} \mu \nu}\right)$ and $\sigma\left(\widetilde{\mathcal{Q}}_{k_{i} \mu \nu A_{i+1}}\right)$.

5.4.2. Spectra $\sigma\left(\widetilde{\mathcal{H}}_{i \mu \nu}\right)$ For any $i=1,2$, the spectra $\sigma\left(\widetilde{\mathcal{H}}_{i \mu \nu}\right)$ for $\mu, \nu=0,1,2,3$ were obtained from exact diagonalization with the unperturbed energy scales $t=\Delta=-\mu=1$. These spectra are characterized by two continua of single-particle excitations separated by an energy gap as is illustrated in Figs. 5(a-d). A discrete number (four) of branches are seen to peel off from these continua, some of which eventually cross the band gap. These midgap branches disperse along the physical boundaries (edges) while they decay exponentially fast away from the edges. They are thus called edge states. Their crossings, if any, define degenerate edge states with group velocities of opposite signs along the edges. Their crossings at vanishing energy and momentum, if any, define degenerate non-propagating zero modes with group velocities of opposite signs along the edges. Their crossings at vanishing energy and non-vanishing momenta, if any, define degenerate propagating zero modes with group velocities of opposite signs along the edges. Their crossings at non-vanishing energies and vanishing momentum, if any, define degenerate non-propagating edge states with group 
velocities of opposite signs along the edges. The entries $\circ$ in the last two columns of Table 3 accounts for the existence of at least one crossing at vanishing energy and momentum. The entries $\otimes$ in the last two columns of Table 3 account for the existence of crossings not necessarily at vanishing energy and momentum. The entries $\times$ in the last two column of Table 3 accounts for the absence of crossings.

The origin of the mid-gap branches in Figs. 5(a-f) is the following. The single-particle Hamiltonian defined in Eq. (5.1b) for a torus geometry supports four boundary states for a cylindrical geometry that are extended along the boundary but exponentially localized away from the boundary due to the non-vanishing Chern numbers for each of $\mathcal{H}_{\boldsymbol{k}}^{(+)}$and $\mathcal{H}_{\boldsymbol{k}}^{(-)}$. These dispersing boundary states show up in the single-particle energy spectrum as four branches of mid-gap states that crosses at vanishing energy and momentum, thereby defining four degenerate non-propagating zero modes. In the presence of the perturbation $0.3 t \mathcal{X}_{13}$ when the boundary dictates that $k_{1}$ is a good quantum number, the energy spectrum shows a fourfold degenerate crossing at vanishing energy and momentum in Fig. 5. (a). However, the very same perturbation, when the boundary dictates that $k_{2}$ is a good quantum number, gaps the unperturbed crossing at vanishing energy and momentum in Fig. 5(b). In the presence of the perturbation $0.3 t \mathcal{X}_{02}$ when the boundary dictates that $k_{2}$ is a good quantum number, the energy spectrum shows a four-fold-degenerate crossing at vanishing energy but away from $k_{2}=0$ in Fig. 5(c)]. In the presence of the perturbation $0.3 t \mathcal{X}_{32}$ when the boundary dictates that $k_{2}$ is a good quantum number, the energy spectrum shows four non-degenerate crossings away from vanishing energy and vanishing momentum in Fig. 5(d). Even though all the crossings in the unperturbed entanglement spectra are robust to the perturbations in Fig. 5. (e-h), they can be shifted away from vanishing energy as in Fig. 5.h) or momentum as in Fig. 5 (g).

To understand the effect of any one of the sixteen perturbations from Table 3 on the zero modes of Hamiltonian $\mathcal{H}_{i}$ defined in Eq. (5.14a), where $i=1,2$ is the choice made along the direction for which periodic boundary conditions is imposed [recall Eq. (5.9)], it is useful to consider Fig. 6. The physical boundaries are the two circles centered at L and $\mathrm{R}$ on the symmetry axis $i+1$ modulo 2 of the cylinder in Fig. 6. In the limit for which the band gap is taken to infinity, the effective theory for the single-particle eigenstates of $\mathcal{H}_{i}$ defined in Eq. (5.14a) that are propagating along the edges $L$ and $R$ with the same group velocity but localized away from them takes the form

$$
\mathcal{H}_{\text {edges } k_{i}}=k_{i} Y_{33} \quad i=1,2 .
$$

Here, we have set the group velocity to unity and

$$
Y_{\mu \nu}:=\rho_{\mu} \otimes \varrho_{\nu}
$$

where we have introduced the two sets of unit $2 \times 2$ matrix and Pauli matrices $\rho_{\mu}$ with $\mu=0,1,2,3$ and $\varrho_{\nu}$ with $\nu=0,1,2,3$. The eigenvalue -1 of $\varrho_{3}$ is interpreted as the left edge $\mathrm{L}$ in Fig. 6. The eigenvalue +1 of $\varrho_{3}$ is interpreted as the right edge $\mathrm{R}$ in Fig. 6. Hence, The matrices $\varrho_{1}$ and $\varrho_{2}$ mix edge states localized on opposite edges. The eigenstates of $\rho_{3}$ describe 
right and left movers on a given edge. The matrices $\rho_{1}$ and $\rho_{2}$ mix left and right movers on a given edge. Hamiltonian (5.16) describes four single-particle states propagating along the two edges with the momentum $\pm k_{i}$. These single-particle states are solely supported on the edges, reason for which we call them edge states. Their direction of propagation originates from the relative sign in $p_{1} \pm \mathrm{i} p_{2}$ if $\mathcal{H}_{i}$ is interpreted as a Bogoliubov-de-Gennes superconductor.

A generic perturbation to the effective Hamiltonian (5.16) that allows mixing between all four edge states is

$$
\mathcal{V}_{\text {edges }}=\sum_{\mu, \nu=0}^{3} v_{\text {edges } \mu \nu} Y_{\mu \nu}, \quad v_{\text {edges } \mu \nu} \in \mathbb{R},
$$

to lowest order in a gradient expansion. For any given $\mu, \nu=0,1,2,3$, the perturbation

$$
\mathcal{V}_{\text {edges } \mu \nu}:=v_{\text {edges } \mu \nu} Y_{\mu \nu}
$$

opens a gap in the spectrum of

$$
\widetilde{\mathcal{H}}_{\text {edges } k_{i} \mu \nu}:=\mathcal{H}_{\text {edges } k_{i}}+\mathcal{V}_{\text {edges } \mu \nu}
$$

if it anti-commutes with $Y_{33}$, thereby forbidding the crossing of the bulk gap by any one of the four mid-gap branches from the effective Hamiltonian (5.16). This is what happens in Fig. 5(b). A perturbation $\mathcal{V}_{\text {edges } \mu \nu}$ that commutes with $Y_{33}$ can shift the location of the two crossings of the four mid-gap branches from the effective Hamiltonian (5.16) away from either vanishing energy or vanishing momentum. For example, the perturbation $\mathcal{V}_{\text {edges } 33}$ with $v_{\text {edges } 33}<0$ moves the crossing in the effective Hamiltonian (5.16) to the right, as happens in Fig.5(c). Figure 5(d) is realized when only one of $v_{\text {edges } 03}$ and $v_{\text {edges } 30}$ are non-vanishing in the perturbation (5.18). Evidently, Fig 5 (a) suggests that not all perturbations parametrized by (5.17) move the degenerate crossing of the effective Hamiltonian Eq. (5.16).

To understand Fig. $5(\mathrm{a})$, we assume that the perturbation $\mathcal{V}_{\text {edges }}$ is local and that the ratio $\xi / L$, where $L$ is the length of the cylinder while $\xi \propto 1 / \Delta$ is the bulk correlation length associated to the bulk gap $\Delta$, is taken to zero. Hence, the effective edge theory for

$$
\widetilde{\mathcal{H}}_{i \mu \nu}:=\mathcal{H}_{i}+\mathcal{V}_{\mu \nu}
$$

remains block diagonal, with one of the block given by

$$
\widetilde{\mathcal{H}}_{\text {edge } k_{i}}=v_{0} \rho_{0}+v_{1} \rho_{1}+v_{2} \rho_{2}+\left(k_{i}+v_{3}\right) \rho_{3}
$$

for some effective real-valued couplings $v_{0}, v_{1}, v_{2}$, and $v_{3}$. As was the case for the bulk Hamiltonian (5.1),

$$
\mathcal{H}_{\text {edge } k_{i}}:=k_{i} \rho_{3}=: \widetilde{\mathcal{H}}_{\text {edge } k_{i} \mu \nu}-\widetilde{\mathcal{V}}_{\text {edge } \mu \nu}
$$

obeys two spectral chiral symmetries, for it anti-commutes with $\rho_{1}$ and $\rho_{2}$. Moreover, $\mathcal{H}_{\text {edge }} k_{i}$ commutes with the operation by which $k_{i} \rightarrow-k_{i}$ is composed with the conjugation of $\mathcal{H}_{\text {edge } k_{i}}$ with either $\rho_{1}$ and $\rho_{2}$. Imposing simultaneously this pair of chiral symmetries and this pair 
of reflection symmetries restricts the effective edge theory to Eq. (5.21b). However, these symmetry constraints are redundant to enforce the form (5.21b) as we now show.

The crossing at vanishing energy and momentum of Hamiltonian $5.21 \mathrm{~b}$ is not generically robust to the perturbations in the perturbed Hamiltonian (5.21a). However, if we impose that the perturbed Hamiltonian (5.21a) anti-commutes with either $\rho_{1}$ or $\rho_{2}$, i.e., the effective perturbed Hamiltonian belongs to the symmetry class AIII, then the edge theory simplifies to the direct sum over blocks of the form

$$
\mathcal{H}_{\text {edge } k_{i} \mu \nu}^{\text {AIII }}=v_{2} \rho_{2}+\left(k_{i}+v_{3}\right) \rho_{3} .
$$

Here, we have chosen, without loss of generality, to implement the chiral symmetry by demanding that $\mathcal{H}_{\text {edge } k_{i} \mu \nu}^{\text {AIII }}$ anti-commutes with $\rho_{1}$. As is, the perturbations in Hamiltonian (5.22) still gaps the unperturbed dispersion (5.21b). However, if we impose the symmetry constraint

$$
\rho_{1} \mathcal{H}_{\text {edge }\left(-k_{i}\right) \mu \nu \mathscr{S} \mathscr{R}} \rho_{1}=\mathcal{H}_{\text {edge }\left(+k_{i}\right) \mu \nu \mathscr{S} \mathscr{R}}
$$

to define $\mathcal{H}_{\text {edge } k_{i} \mu \nu \mathscr{S} \mathscr{R}}$, we find that

$$
\mathcal{H}_{\text {edge } k_{i} \mu \nu \mathscr{S} \mathscr{R}}=k_{i} \rho_{3} .
$$

In other words, if we impose, in addition to the chiral symmetry, the reflection symmetry generated by $\rho_{1}$ and $k_{i} \rightarrow-k_{i}$, we find that no perturbation can gap the unperturbed dispersion (5.21b). Had we chosen to impose the reflection symmetry generated by $\rho_{2}$ instead of $\rho_{1}$, i.e., a realization of parity that anti-commutes with the choice $\rho_{1}$ we made to implement the chiral transformation, then the perturbation $v_{2} \rho_{2}$ in Eq. (5.22) would not be prevented from removing the crossing of the edge states through a spectral gap.

Now, the reflection symmetry obeyed by the effective edge Hamiltonian 5.21b can only originate from a reflection about the plane frame in blue that contains the cylinder axis in Fig. 6. A reflection about the plane framed in red that is orthogonal to the cylinder axis in Fig. 6 exchanges the edges $L$ and $R$. As such, it can only be represented within the representation defined by Eqs. (5.16) and (5.17) of the effective edge Hamiltonian that allows mixing of the two opposite edges.

5.4.3. Spectra $\sigma\left(\widetilde{\mathcal{Q}}_{\mu \nu A_{i+1}}\right)$ For any $i=1,2$, the spectra $\sigma\left(\widetilde{\mathcal{Q}}_{\mu \nu A_{i+1}}\right)$ for $\mu, \nu=0,1,2,3$ were also obtained from exact diagonalization with the unperturbed energy scales $t=\Delta=$ $-\mu=1$. These spectra are characterized by two nearly flat bands at \pm 1 from which pairs of mid-gap branches occasionally peel off as is illustrated in Figs. 5(e-f). We observe in both Figs. 5(e) and 5(f) one crossing of the mid-gap branches at vanishing entanglement eigenvalue and momentum. The crossing in $5 \mathrm{~g}$ ) takes place at vanishing entanglement eigenvalue but non-vanishing momentum. The crossing in $5(\mathrm{~h})$ takes place at non-vanishing entanglement but vanishing momentum. Crossings of the mid-gap branches taking place at vanishing entanglement eigenvalue and momentum are indicated by $\circ$ in the last two columns of Table 3 . The absence of any crossing of the mid-gap branches is indicated by $\times$ in the last two columns 


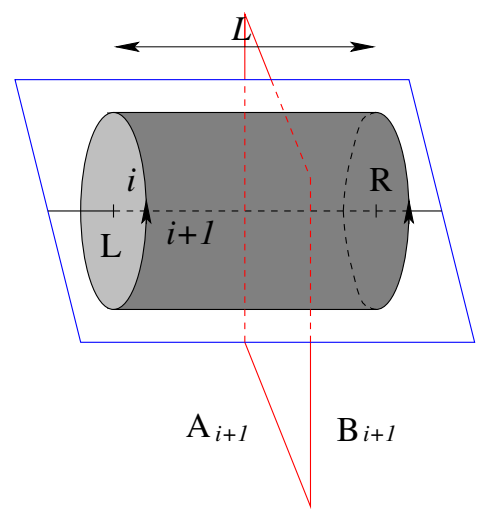

Figure 6. (Color online) A cylinder of length $L$ along the $i+1$ modulo 2 direction, while the coordinate $i=1,2$ has been compactified. We define two reflection planes. The first is defined by the blue frame that includes the cylinder axis. The second is defined by the red frame normal to the cylinder axis and intersecting the cylinder axis at its mid-point. The circles centered at the point $\mathrm{L}$ and $\mathrm{R}$ on the cylinder axis are the two disconnected boundaries of the cylinder. The reflection about the plane framed in blue leaves each circle invariant as a set. The reflection about the plane framed in red exchanges the circles centered on the cylinder axis at $\mathrm{L}$ and $\mathrm{R}$. This plane framed in red defines the entangling boundary in a cylindrical geometry as defined by the partition 5.10$)$.

of Table 3 . Crossings of the mid-gap branches taking place away from vanishing entanglement eigenvalue and momentum are indicated by $\otimes$ in the last two columns of Table 3 .

Comparison of Figs. 5(a-d) and Figs. 5(e-f) suggests that the existence of crossings in the entanglement spectrum defined by Eq. (5.14b) is more robust to perturbations than that for the Hamiltonian spectrum. Choose $i=1,2(i+1$ modulo 2$), \mu=0,1,2,3$, and $\nu=0,1,2,3$. According to Table 3 any crossing in the perturbed Hamiltonian spectrum $\sigma\left(\widetilde{\mathcal{H}}_{i \mu \nu}\right)$ implies a crossing in the perturbed entanglement spectrum $\sigma\left(\widetilde{\mathcal{Q}}_{\mu \nu A_{i+1}}\right)$. The converse is not true. There are crossings in the perturbed entanglement spectrum $\sigma\left(\widetilde{\mathcal{Q}}_{\mu \nu A_{i+1}}\right)$ but no crossings in the perturbed Hamiltonian spectrum $\sigma\left(\widetilde{\mathcal{H}}_{i \mu \nu}\right)$, as is shown explicitly when comparing Fig. 5 (f) to Fig. 5(b). In fact, Table 3 shows the following difference between the perturbed Hamiltonian and entanglement spectra. On the one hand, the existence of crossings in $\sigma\left(\widetilde{\mathcal{H}}_{i \mu \nu}\right)$ does not necessarily implies the existence of crossings in $\sigma\left(\widetilde{\mathcal{H}}_{(i+1) \mu \nu}\right)$. On the one hand, the existence of crossings in $\sigma\left(\widetilde{\mathcal{Q}}_{\mu \nu A_{i}}\right)$ implies the existence of crossings in $\sigma\left(\widetilde{\mathcal{Q}}_{\mu \nu A_{i+1}}\right)$ and vice versa.

To understand the effect of any one of the sixteen perturbations from Table 3 on the zero modes of $\mathcal{Q}_{A_{i+1}}$ defined in Eq. (5.14b), where $i+1$ modulo 2 is the choice made for the direction along which the partition is made [recall Eq. [5.9]], it is useful to consider the compactifcation along the cylinder axis of Fig. 6 consisting in identifying the circles at $\mathrm{L}$ and R. We thereby obtain a torus, whose intersection with the plane framed in red from Fig. 6 defines two entangling boundaries separated by the distance $L / 2$. The spectrum $\sigma\left(\mathcal{Q}_{A_{i+1}}\right)$ supports four mid-gap branches, two per entangling boundaries. In the limit for which the band gap is taken to infinity, the effective theory for the eigenstates of $\mathcal{Q}_{A_{i+1}}$ that propagate 
along the entangling boundaries but are localized away from them is given by

$$
\mathcal{Q}_{\text {edges } k_{i} A_{i+1}}=k_{i} Y_{33}
$$

The interpretation of the $4 \times 4$ matrices $Y_{\mu \nu}$ with $\mu, \nu=0,1,2,3$ is the same as the one given below Eq. (5.16b).

A generic perturbation to the effective Hamiltonian (5.25) that allows mixing between all four entangling states is

$$
\delta \mathcal{Q}_{\text {edges } A_{i+1}}=\sum_{\mu, \nu=0}^{3} v_{\operatorname{edges~} \mu \nu A_{i+1}} Y_{\mu \nu}, v_{\operatorname{edges} \mu \nu A_{i+1}} \in \mathbb{R},
$$

to lowest order in a gradient expansion. For any given $\mu, \nu=0,1,2,3$, the perturbation

$$
\delta \mathcal{Q}_{\text {edges } \mu \nu A_{i+1}}:=v_{\text {edges } \mu \nu A_{i+1}} Y_{\mu \nu}
$$

opens a gap in the spectrum of

$$
\widetilde{\mathcal{Q}}_{\text {edges } k_{i}} \mu \nu A_{i+1}:=\mathcal{Q}_{\text {edges } k_{i} A_{i+1}}+\delta \mathcal{Q}_{\text {edges } \mu \nu A_{i+1}}
$$

if it anti-commutes with $Y_{33}$, thereby forbidding the crossing of the bulk gap by any one of the four mid-gap branches from the effective Hamiltonian 5.25 . A perturbation $\mathcal{V}_{\text {edges } \mu \nu A_{i+1}}$ that commutes with $Y_{33}$ can shift the location of the two crossings of the four mid-gap branches from the effective Hamiltonian (5.25) away from either vanishing energy or vanishing momentum.

To understand the robustness of the effective 5.25 to perturbations, we assume that the perturbation $\mathcal{V}_{\text {edges } A_{i+1}}$ is local and that the ratio $\xi / L$, where $L$ is the length of the cylinder while $\xi \propto 1 / \Delta$ is the bulk correlation length associated to the bulk gap $\Delta$, is taken to zero. Hence, the effective edge theory for

$$
\widetilde{\mathcal{Q}}_{\mu \nu A_{i+1}}:=\mathcal{Q}_{A_{i+1}}+\delta \mathcal{Q}_{\mu \nu A_{i+1}}
$$

remains block diagonal, with one of the block given by

$$
\widetilde{\mathcal{Q}}_{\text {edge } k_{i} A_{i+1}}=v_{0} \rho_{0}+v_{1} \rho_{1}+v_{2} \rho_{2}+\left(k_{i}+v_{3}\right) \rho_{3}
$$

for some effective real-valued couplings $v_{0}, v_{1}, v_{2}$, and $v_{3}$.

To proceed, we recall that if $\mathcal{S}$ is a unitary spectral symmetry of $\mathcal{H}$ in that $\mathcal{S}^{-1} \mathcal{H} \mathcal{S}=-\mathcal{H}$ and if $\mathcal{S}$ is block diagonal with respect to the partition into $A$ and $B$, we then deduce from

$$
\begin{aligned}
\mathcal{Q} & =\mathcal{S}^{-1}(-1) \mathcal{Q} \mathcal{S} \\
& =\left(\begin{array}{cc}
S_{A}^{-1} & 0 \\
0 & S_{B}^{-1}
\end{array}\right)\left(\begin{array}{cc}
-Q_{A} & +2 C_{A B} \\
+2 C_{B A} & -Q_{B}
\end{array}\right)\left(\begin{array}{cc}
S_{A} & 0 \\
0 & S_{B}
\end{array}\right)
\end{aligned}
$$

that

$$
S_{A}^{-1} Q_{A} S_{A}=-Q_{A}, \quad S_{B}^{-1} Q_{B} S_{B}=-Q_{B},
$$


and

$$
S_{A}^{-1} C_{A B} S_{B}=-C_{A B}, \quad S_{B}^{-1} C_{B A} S_{A}=-C_{B A} .
$$

As was the case for the bulk Hamiltonian (5.1),

$$
\mathcal{Q}_{\text {edge } k_{i} A_{i+1}}:=k_{i} \rho_{3}
$$

obeys two spectral chiral symmetries, for it anti-commutes with $\rho_{1}$ and $\rho_{2}$, and two spectral symmetries under charge conjugation, for it anti-commutes with the composition of $k_{i} \rightarrow-k_{i}$ with conjugation by $\rho_{0}$ or $\rho_{3}$.

The crossing at vanishing energy in Eq. (5.33) is not generically robust to the perturbations in Eq. (5.30). However, if we impose that Eq. (5.30) anti-commutes with either $\rho_{1}$ or $\rho_{2}$, then the entangling theory simplifies to the direct sum over blocks of the form

$$
\mathcal{Q}_{\text {edge } k_{i} \mu \nu A_{i+1}}^{\text {AIII }}=v_{2} \rho_{2}+\left(k_{i}+v_{3}\right) \rho_{3} .
$$

Here and without loss of generality, we have chosen to implement the chiral symmetry by demanding that $\mathcal{Q}_{\text {edge } k_{i} \mu \nu A_{i+1}}^{\mathrm{AIII}}$ anti-commutes with $\rho_{1}$. As is, one perturbation in Hamiltonian (5.34) still gaps the unperturbed dispersion (5.33). However, if we impose the symmetry constraint

$$
\rho_{2} \widetilde{\mathcal{Q}}_{\text {edge } k_{i} \mu \nu A_{i+1} \mathscr{S} \mathscr{R}_{i}} \rho_{2}=\widetilde{\mathcal{Q}}_{\text {edge } k_{i} \mu \nu A_{i+1} \mathscr{S} \mathscr{R}_{i}}
$$

to define $\widetilde{\mathcal{Q}}_{\text {edge } k_{i} \mu \nu A_{i+1}} \mathscr{S} \mathscr{R}_{i}$, we find that

$$
\widetilde{\mathcal{Q}}_{\text {edge } k_{i} \mu \nu A_{i+1} \mathscr{S} \mathscr{R}_{i}}=\left(k_{i}+v_{3}\right) \rho_{3}
$$

displays a crossing at vanishing energy and momentum $k_{i}=-v_{3}$. In other words, if we impose, in addition to the chiral symmetry, the effective chiral symmetry generated by $\rho_{2}$, we find that no perturbation can gap the unperturbed dispersion (5.33), although it can move the momentum of the zero mode away from vanishing energy. As is implied by the notation, the effective chiral symmetry generated by $\rho_{2}$ originates from protecting the symmetry class AIII by demanding that reflection symmetry about the plane defining the entangling boundaries, i.e., the plane framed in red in Fig. 6, holds. According to Sec. 3.2 the reflection about the plane framed in red in Fig. 6 induces a local effective chiral symmetry of the form given in Eq. (2.45), i.e., there exists a

$$
\Gamma_{\mathscr{R}_{i}}:=C_{A_{i+1} B_{i+1} k_{i}} \mathcal{R}_{i}
$$

such that

$$
\left\{\Gamma_{\mathscr{R}_{i}}, \widetilde{\mathcal{Q}}_{\text {edge } k_{i} \mu \nu A_{i+1}} \mathscr{S} \mathscr{R}_{i}\right\}=0 \text {. }
$$

The choice to represent $\Gamma_{\mathscr{R}}$ by $\rho_{2}$, given the choice to represent the chiral symmetry $\mathscr{S}$ by $\rho_{1}$, is a consequence of the following assumption and the following fact. First, we demand that $\mathscr{S}$ and $\mathscr{R}_{i}$ commute, i.e., we demand that

$$
\left[\mathcal{S}, \mathcal{R}_{i}\right]=0
$$


Second, the identity

$$
\begin{aligned}
\left\{\mathcal{S}, \mathcal{R}_{i}\right\} & =\left\{\mathcal{S}, C_{A_{i+1} B_{i+1} k_{i}} \mathcal{R}_{i}\right\} \\
& =\left\{\mathcal{S}, C_{A_{i+1} B_{i+1} k_{i}}\right\} \mathcal{R}_{i}-C_{A_{i+1} B_{i+1} k_{i}}\left[\mathcal{S}, \mathcal{R}_{i}\right]
\end{aligned}
$$

holds.

5.4.4. Spectra $\sigma\left(\mathcal{H}_{k_{i} \mathscr{R} ; \mathscr{S}}\right)$ and $\sigma\left(Q_{k_{i} \mathscr{R} ; \mathscr{S} A_{i+1}}\right)$ It is time to present the reasoning that delivers Table 4. Let $i=1,2$ and define $i+1$ modulo 2 . We assume that the underlying microscopic model has the doublet of symmetries $(\mathscr{R} ; \mathscr{S}) \sim\left(\mathcal{R}_{\mu \nu}, \mathcal{S}_{\mu \nu}\right)$ where $\mathscr{S} \sim \mathcal{S}_{\mu \nu}$ defines the symmetry class AIII. We denote the most general perturbation that is compliant with the doublet of symmetries $(\mathscr{R} ; \mathscr{S})$ defining a given row of Table 2 by $\mathcal{V}_{\mathscr{R}: \mathscr{S}}$. The explicit form of this perturbation is to be found in the second column of Table 4 as one varies $(\mathscr{R} ; \mathscr{S})$. For a given row in Table $4, \mathcal{V}_{\mathscr{R} ; \mathscr{S}}$ is contained in the most general perturbation $\mathcal{V}_{\mathscr{S}}$ that is compliant with the AIII symmetry. We define the single-particle Hamiltonian $\mathcal{H}_{i \mathscr{R} ; \mathscr{S}} \equiv \mathcal{H}_{i}+\mathcal{V}_{\mathscr{R} ; \mathscr{S}}$ by its matrix elements

$$
\begin{aligned}
& \mathcal{H}_{k_{i} \mathscr{R} ; \mathscr{S} r_{i+1}, r_{i+1}^{\prime}}:=\mathcal{H}_{k_{i} r_{i+1}, r_{i+1}^{\prime}}+\mathcal{V}_{\mathscr{R} ; \mathscr{S} r_{i+1}, r_{i+1}^{\prime}}, \\
& \mathcal{V}_{\mathscr{R} ; \mathscr{S} r_{i+1}, r_{i+1}^{\prime}}:=\delta_{r_{i+1}, r_{i+1}^{\prime}} \sum_{\mu, \nu \in \text { row }} v_{\mu \nu} \mathcal{X}_{\mu \nu} .
\end{aligned}
$$

The corresponding equal-time one-point correlation matrix is $\mathcal{Q}_{\mathscr{R} ; \mathscr{S}(i+1)}$ and its upperleft block is $Q_{\mathscr{R} ; \mathscr{S} A_{i+1}}$. The third column in Table 4 provides one sign for each row. The sign $\eta_{\mathscr{S}}$ is positive if $\mathscr{R}$ commutes with $\mathscr{S}$ and negative if $\mathscr{R}$ anti-commutes with $\mathscr{S}$. The information contained in $\eta_{\mathscr{S}}$ is needed to read from Table VI of Ref. [16] the bulk topological index of the single-particle Hamiltonian (5.41). This topological index does not guarantee that $\mathcal{H}_{\mathscr{R} ; \mathscr{S}}$ supports boundary states in an open geometry. On the other hand, $Q_{\mathscr{R} ; \mathscr{S} A_{i+1}}$ supports boundary states on the entangling boundaries. The entries $\circ$ for the columns $\sigma\left(H_{k_{i} \mathscr{R} ; \mathscr{P}}\right)$ and $\sigma\left(Q_{k_{i} \mathscr{R} ; \mathscr{S} A_{i+1}}\right)$ are a consequence of our stability analysis. On the one hand, Table 4 demonstrates explicitly that the presence of a reflection symmetry in addition to the chiral symmetry does not guarantee that a non-vanishing bulk topological index implies protected edge states in the spectrum $\sigma\left(H_{k_{i} \mathscr{R} ; \mathscr{S}}\right)$ for both edges $i=1,2$. On the other hand, Table 4 demonstrates explicitly that a non-vanishing bulk topological index always implies protected entangling states in the spectrum $\sigma\left(Q_{k_{i} \mathscr{R} ; \mathscr{S} A_{i+1}}\right)$ irrespective of the choice $i=1,2$ made for the entangling boundary.

\subsection{Existence of spectral flows in the entanglement spectra}

We are going to study the dependences of the spectra $\sigma\left(\mathcal{H}_{k_{i} \mathscr{R} ; \mathscr{S}}\right)$ and $\sigma\left(Q_{k_{i} \mathscr{R} ; \mathscr{P} A_{i+1}}\right)$ (with $i+1$ defined modulo 2) on the system sizes for the single-particle Hamiltonian $\mathcal{H}_{k_{i}: \mathscr{R} ; \mathscr{S}}$ defined by the matrix elements $(5.41)$ and the corresponding upper-left block $Q_{k_{i} \mathscr{R} ; \mathscr{I}} A_{i+1}$ from the equal-time one-point correlation matrix. Without loss of generality, we will present 


\begin{tabular}{|c|c|c|c|c|c|c|c|c|}
\hline \multicolumn{2}{|c|}{ Symmetries in class AIII } & Generic perturbation $\mathcal{V}_{\mathscr{R} ; \mathscr{S}}$ & $\eta_{\mathscr{S}}$ & Index & $\sigma\left(\mathcal{H}_{k_{1} \mathscr{R} ; \mathscr{S}}\right)$ & $\sigma\left(Q_{k_{1} \mathscr{R} ; \mathscr{S} A_{2}}\right)$ & $\sigma\left(\mathcal{H}_{k_{2} \mathscr{R} ; \mathscr{S}}\right)$ & $\sigma\left(Q_{k_{2} \mathscr{R} ; \mathscr{S} A_{1}}\right)$ \\
\hline \multirow{4}{*}{$\left(\mathscr{R}_{1}, \mathscr{S}\right)$} & $\mathcal{R}_{13}, \mathcal{S}_{11}$ & $v_{03} \mathcal{X}_{03}+v_{13} \mathcal{X}_{13}+v_{21} \mathcal{X}_{21}+v_{31} \mathcal{X}_{31}$ & - & 0 & $\times$ & $x$ & $\times$ & $\times$ \\
\hline & $\mathcal{R}_{13}, \mathcal{S}_{21}$ & $v_{03} \mathcal{X}_{03}+v_{10} \mathcal{X}_{10}+v_{22} \mathcal{X}_{22}+v_{31} \mathcal{X}_{31}$ & + & $\mathbb{Z}$ & $\times$ & $\odot$ & o & $\otimes$ \\
\hline & $\mathcal{R}_{23}, \mathcal{S}_{11}$ & $v_{03} \mathcal{X}_{03}+v_{12} \mathcal{X}_{12}+v_{20} \mathcal{X}_{20}+v_{31} \mathcal{X}_{31}$ & + & $\mathbb{Z}$ & $\times$ & $\odot$ & $\circ$ & $\otimes$ \\
\hline & $\mathcal{R}_{23}, \mathcal{S}_{21}$ & $v_{03} \mathcal{X}_{03}+v_{11} \mathcal{X}_{11}+v_{23} \mathcal{X}_{23}+v_{31} \mathcal{X}_{31}$ & - & 0 & $\times$ & $\times$ & $\times$ & $\times$ \\
\hline \multirow{4}{*}{$\left(\mathscr{R}_{2}, \mathscr{S}\right)$} & $\mathcal{R}_{10}, \mathcal{S}_{11}$ & $v_{02} \mathcal{X}_{02}+v_{03} \mathcal{X}_{03}+v_{12} \mathcal{X}_{12}+v_{13} \mathcal{X}_{13}$ & + & $\mathbb{Z}$ & $\circ$ & $\otimes$ & $\times$ & $\odot$ \\
\hline & $\mathcal{R}_{10}, \mathcal{S}_{21}$ & $v_{02} \mathcal{X}_{02}+v_{03} \mathcal{X}_{03}+v_{10} \mathcal{X}_{10}+v_{11} \mathcal{X}_{11}$ & - & 0 & $\times$ & $\times$ & $x$ & $\times$ \\
\hline & $\mathcal{R}_{20}, \mathcal{S}_{11}$ & $v_{02} \mathcal{X}_{02}+v_{03} \mathcal{X}_{03}+v_{20} \mathcal{X}_{20}+v_{21} \mathcal{X}_{21}$ & - & 0 & $\times$ & $\times$ & $x$ & $\times$ \\
\hline & $\mathcal{R}_{20}, \mathcal{S}_{21}$ & $v_{02} \mathcal{X}_{02}+v_{03} \mathcal{X}_{03}+v_{22} \mathcal{X}_{22}+v_{23} \mathcal{X}_{23}$ & + & $\mathbb{Z}$ & ○ & $\otimes$ & $\times$ & $\odot$ \\
\hline
\end{tabular}

Table 4. The first four rows of the second column give all possible doublets of generators consisting of a reflection about the direction 1 and a chiral transformation. The last four rows of the second column give all possible doublets of generators consisting of a reflection about the direction 2 and a chiral transformation. The third column gives for each row the most general perturbation $\mathcal{V}_{\mathscr{R} ; \mathscr{S}}$ that commutes with the operation of reflection and anticommutes with the operation of chirality. The fourth column gives the sign $\eta_{\mathscr{S}}$ defined by $\mathcal{R} \mathcal{S} \mathcal{R}=\eta_{\mathscr{S}} \mathcal{S}$. The fifth column is an application of the classification for the symmetryprotected topological band insulators in two-dimensional spaces derived in Refs. [15] and [16] (Table VI from Ref. [16] was particularly useful). The topological indices $\mathbb{Z}$ and 0 correspond to topologically nontrivial and trivial bulk phases, respectively. The entries $\circ$ or $\times$ in the last two columns denote the presence or absence, respectively, of zero modes in the spectra of $\sigma\left(\mathcal{H}_{k_{1} \mathscr{R} ; \mathscr{S}}\right)$, on the one hand, and $\sigma\left(\mathcal{H}_{k_{2} \mathscr{R} ; \mathscr{S}}\right)$, on the other hand. Whereas $\times$ denotes the absence of zero modes, the entries $\odot$ or $\otimes$ in the last two columns denote the presence of zero modes with or without spectral flow, respectively, in the spectra of $\sigma\left(\widetilde{Q}_{k_{1} \mathscr{R} ; \mathscr{S} A_{2}}\right)$, on the one hand, and $\sigma\left(\widetilde{Q}_{k_{2} \mathscr{R} ; \mathscr{S} A_{1}}\right)$, on the other hand.

numerical results obtained by choosing the fifth row in Table 4 to define $\mathcal{H}_{k_{i} \mathscr{R}_{2} ; \mathscr{S}}$ and $Q_{k_{i} \mathscr{R}_{2} ; \mathscr{S} A_{i+1}}$ with $\left(v_{02}, v_{03}, v_{12}, v_{13}\right)=(0.2,-0.1,0.05,0.3)$.

The question we want to address is the following. On the one hand, Figs. 5(a-d) suggest that the mid-gap branches merge into the continuum when $\pi / 2<\left|k_{i}\right|<\pi$. On the other hand, the mid-gap branches in Fig. 5(e) seem to be separated by a very small gap from all other bands in the vicinity of $\left|k_{1}\right|=\pi$, while no gap is resolved in energy between the midgap branches and all other branches in the vicinity of $\left|k_{1}\right|=\pi$ for Figs. 5 (f-h).

The first question we are going to address is how to interpret the "peeling of" the miggap branches from the continua in Figs. $5(\mathrm{a}-\mathrm{d})$. To this end, we present in Fig 7 (a) the spectrum $\sigma\left(\mathcal{H}_{k_{1} \mathscr{R}_{2} ; \mathscr{S}}\right)$ obtained by imposing periodic boundary conditions along the direction $i=1$ and open boundary conditions along the direction $i+1=2$ for the linear system sizes $M_{1}=128$ and $M_{2}=64$. We then compute the spectrum of $\mathcal{H}_{k_{1} \mathscr{R}_{2} ; \mathscr{S}}$ for each value $M_{2}=16,32,48,64,80,96$ of the cylinder height. At last, we compute the minimal value $\Delta_{\min }\left(\pi / 2, M_{2}\right)>0$ taken by the difference in energy between the positive energy eigenvalues at $k_{1}=\pi / 2$ from any one of the bulk bands and the mig-gap branch with positive energy eigenvalue at $k_{1}=\pi / 2$. The dependence of this direct gap $\Delta_{\min }\left(\pi / 2, M_{2}\right)>0$ on $M_{2}=16,32,48,64,80,96$ holding $M_{1}=128$ fixed is plotted in Fig 7(b). The fast decrease of $\Delta_{\text {min }}\left(\pi / 2, M_{2}\right)>0$ with increasing values of $M_{2}=16,32,48,64,80,96$ holding $M_{1}=128$ fixed is interpreted as the merging of the mid-gap branch into the continuum of conduction bulk states in the quasi-one-dimensional limit $M_{2} \rightarrow \infty$ holding $M_{1}=128$ and $\pi / 2 \leq k_{1} \leq \pi$ 
(a)

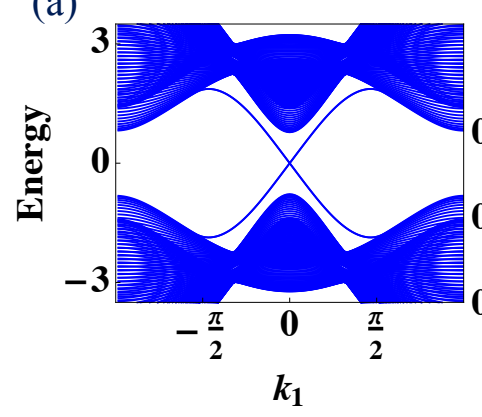

(d)

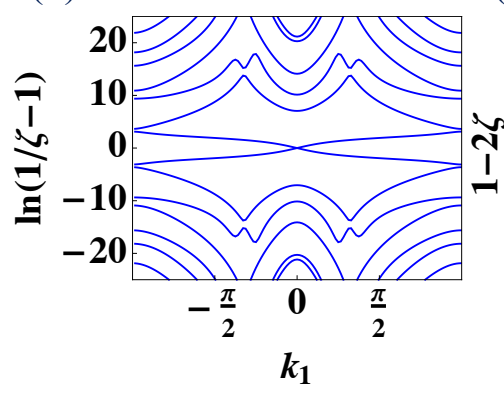

(b)

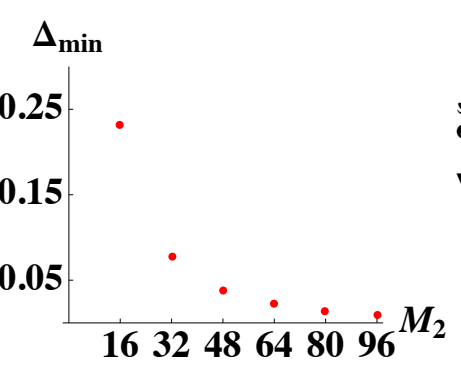

(e)

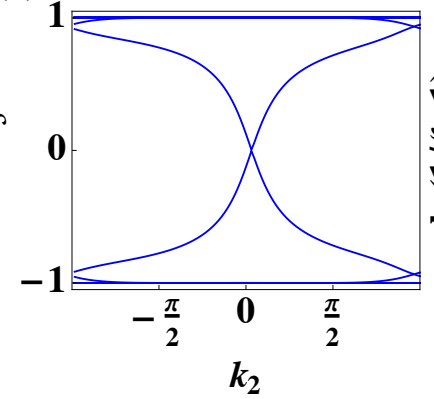

(c)

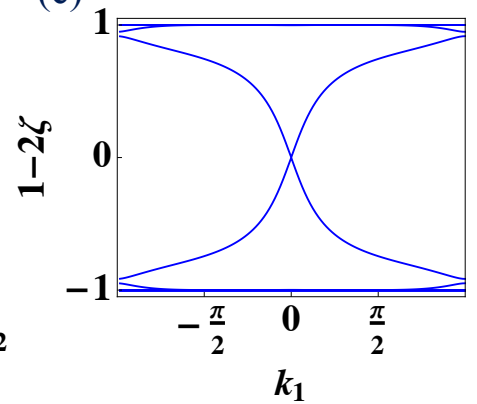

(f)

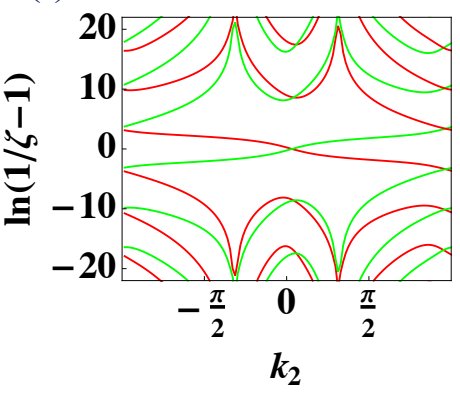

Figure 7. (Color online) Spectra $\sigma\left(\mathcal{H}_{k_{i} \mathscr{R}_{2} ; \mathscr{S}}\right), \sigma\left(Q_{k_{i} \mathscr{R}_{2} ; \mathscr{S} A_{i+1}}\right)$, and $\sigma\left(\ln \left(Q_{k_{i} \mathscr{R}_{2} ; \mathscr{S} A_{i+1}-}^{-1}\right.\right.$ 1)) (with $i+1$ defined modulo 2) for the single-particle Hamiltonian $\mathcal{H}_{k_{i} \mathscr{R}_{2} ; \mathscr{S}}$ defined by the matrix elements 5.41) and the corresponding upper-left block $Q_{k_{i} \mathscr{R}_{2} ; \mathscr{S} A_{i+1}}$ from the equaltime one-point correlation matrix with $\left(v_{02}, v_{03}, v_{12}, v_{13}\right)=(0.2,-0.1,0.05,0.3)$ taken from the fifth line from Table 4 . (a) Spectrum $\sigma\left(\mathcal{H}_{k_{1} \mathscr{R}_{2} ; \mathscr{S}}\right)$ for the linear sizes $M_{1}=128$ and $M_{2}=$ 64. (b) Scaling of the direct gap between the conduction bands at the momentum $k_{1}=\pi / 2$ and the mid-gap branch with positive energy eigenvalue at the momentum $k_{1}=\pi / 2$ as a function of increasing $M_{2}=16,32,64,80,96$ holding $M_{1}=128$ fixed. (c) Spectrum $Q_{k_{1} \mathscr{R}_{2} ; \mathscr{S} A_{2}}$ for the linear sizes $M_{1}=128$ and $M_{2}=64$. (d) Spectrum $\sigma\left(\ln \left(Q_{k_{1} \mathscr{R}_{2} ; \mathscr{S} A_{2}}^{-1}-1\right)\right)$ for the linear sizes $M_{1}=128$ and $M_{2}=64$. (e) Spectrum $Q_{k_{2} \mathscr{R}_{2} ; \mathscr{S} A_{1}}$ for the linear sizes $M_{1}=64$ and $M_{2}=128$. (f) Spectrum $\sigma\left(\ln \left(Q_{k_{2}}^{-1} \mathscr{R}_{2} ; \mathscr{S} A_{1}-1\right)\right)$ for the linear sizes $M_{1}=64$ and $M_{2}=128$. The coloring follows from the existence of the operator 5.43 that commutes with $Q_{k_{2} \mathscr{R}_{2} ; \mathscr{S} A_{1}}$. The coloring demonstrates the existence of a spectral flow that connects the valence to the conduction continua through the mid-gap branches in the thermodynamic limit $M_{1}, M_{2} \rightarrow \infty$.

fixed. Similarly, one may verify that the mig-gap branches merge into the valence and conduction bulk continua in Figs. 5(a-d) for a non-vanishing interval of momenta $k_{i}$ in the quasi-one-dimensional limit $M_{i+1} \rightarrow \infty$ holding $M_{i}$ fixed. If we change the conserved momentum $k_{i}$ adiabatically, say by imposing twisted boundary conditions instead of periodic ones along the $i$ direction, a charge can be transferred from the valence bulk continuum to the conduction bulk continuum through the mid-gap branches. This is an example of spectral flow induced by mid-gap states crossing a bulk gap.

The same quasi-one-dimensional scaling analysis, when performed on the spectra $\sigma\left(\mathcal{H}_{k_{2} \mathscr{R}_{2} ; \mathscr{S}}\right)$ and $\sigma\left(Q_{k_{1} \mathscr{R}_{2} ; \mathscr{S} A_{2}}\right)$, delivers a very different result. First, the mid-gap branches of $\mathcal{H}_{k_{2} \mathscr{R}_{2} ; \mathscr{S}}$ fail to cross. Second, the minimal value $\Delta_{\text {min }}\left(\pi, M_{2}\right)>0$ taken by the difference between the positive eigenvalues at $k_{1}=\pi$ from any one of the bulk bands and the mig-gap 
branch with positive energy eigenvalues at $k_{1}=\pi$ converge to a non-vanishing value upon increasing $M_{2}=16,32,48,64,80,96$ holding $M_{1}=128$ fixed in Fig 7(c). Instead of plotting the evidence for the saturation value $\lim _{M_{2} \rightarrow \infty} \Delta_{\text {min }}\left(\pi, M_{2}\right)>0$, we plot in Fig $7(\mathrm{~d})$ the entanglement spectrum

$$
\varpi_{k_{1}}:=\ln \left(\frac{1}{\zeta_{k_{1}}}-1\right)
$$

which we already encountered in Eq. (2.13).

We now reproduce Figs 7(c) and Fig 7(d) for $M_{1}=64$ and $M_{2}=128$ with the single change that we choose the geometry in which it is $k_{2}$ instead of $k_{1}$ that is chosen to be the good quantum number. There follows Figs 7 (e) and Fig 7 (f) for $\sigma\left(Q_{k_{2} \mathscr{R}_{2} ; \mathscr{S}_{1}}\right)$ and $\sigma\left(\ln \left(Q_{k_{2} \mathscr{R}_{2} ; \mathscr{S} A_{1}}^{-1}-1\right)\right)$, respectively. We have verified that, if we increase simultaneously $M_{1}$ and $M_{2}$, there is a level crossing between the mid-gap branches and the bulk branches in the neighborhood of $\pm \pi$. The existence of this level crossing is implied by the coloring of the bands in Fig 7(f). This coloring has the following origin.

For any row from Table 4, we may construct the operator

$$
\Lambda_{k_{i} \mathscr{R} A_{i+1}}:=\Gamma_{k_{i} \mathscr{R}} S_{A_{i+1}}
$$

whenever the reflection $\mathscr{R}$ is block off-diagonal with respect to the partition. Here, $\Gamma_{k_{i} \mathscr{R}}$ was defined in Eq. (2.45), while the representation $\mathcal{S}$ of the chiral operation $\mathscr{S}$ is diagonal in the partition with the upper-left block $S_{A_{i+1}}$.

For the fifth row from Table $4, \Gamma_{k_{1} \mathscr{R}_{2}}$ does not exist in the geometry that defines $Q_{k_{1} \mathscr{R}_{2} ; \mathscr{S} A_{2}}$, since $\mathscr{R}_{2}$ is block diagonal for this partition. For the fifth row from Table $4, \Gamma_{k_{2}} \mathscr{R}_{2}$ exists in the geometry that defines $Q_{k_{2} \mathscr{R}_{2} ; \mathscr{S} A_{1}}$ and anti-commutes with $Q_{k_{2} \mathscr{R}_{2} ; \mathscr{S} A_{1}}$ according to Eq. 2.52, since $\mathscr{R}_{2}$ is block off-diagonal for this partition. Hence, $\Lambda_{k_{2} \mathscr{R}_{2} A_{1}}$ commutes with

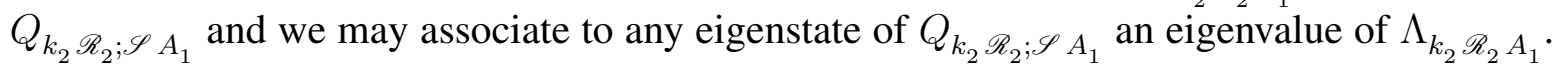
This eigenvalue of $\Lambda_{k_{2} \mathscr{R}_{2} A_{1}}$ is complex valued as $\Lambda_{k_{2} \mathscr{R}_{2} A_{1}}$ is not necessarily Hermitian. It turns out that $\Lambda_{k_{2} \mathscr{R}_{2} A_{1}}$ is purely imaginary according to our exact diagonalizations. The coloring in Fig 7 (f) corresponds to the sign of the imaginary eigenvalue of $\Lambda_{k_{2} \mathscr{R}_{2} A_{1}}$. This quantum number rules out an avoided level crossing of the mid-gap branches in the neighborhood of $k_{2}=0$ in Fig 7 (f). This quantum number also rules out an avoided level crossing at the zone boundary $k_{2}=\pi$. Inspection of the colored dispersions in Fig 7 (f) suggests that twisting boundary conditions induces a spectral flow from the valence to the conduction bands through the mid-gap branches. This is the basis of the distinction between crossings of mid-gap branches that are compatible with a spectral flow and are denoted by $\odot$ in Table 4 and crossing of mid-gap branches that are not compatible with a spectral flow and are denoted by $\otimes$ in Table 4 .

In summary, we can understand the fifth row from Table 4(an all remaining rows with the same reasoning) as follows. Because the reflection and chiral operations commute, the topological index of a generic Hamiltonian obeying these two symmetries belongs to $\mathbb{Z}$. Protected edge states can but need not be present in the spectrum of the Hamiltonian in a cylindrical geometry. Protected edge states arise if and only if the disconnected boundaries 
are not mixed by the reflection symmetry, i.e., for $\mathcal{H}_{k_{1} \mathscr{R}_{2} ; \mathscr{S}}$ but not for $\mathcal{H}_{k_{2} \mathscr{R}} ; \mathscr{S}$. On the other hand, the upper-left block $Q_{k_{1} \mathscr{R}_{2} ; \mathscr{S} A_{2}}$ and $Q_{k_{2} \mathscr{R}_{2} ; \mathscr{S} A_{1}}$ of the equal-time correlation matrix both have robust mid-gap branches that cross. However, it is only the mid-gap branches of $Q_{k_{2} \mathscr{R _ { 2 }} ; \mathscr{S} A_{1}}$ that support a spectral flow between the valence and conduction continua, for it is only then that the reflection $\mathscr{R}_{2}$ is block off-diagonal in the partition defined by $A_{1}$. The fifth row from Table 4 demonstrates that topological protection can be absent from the spectrum of the Hamiltonian but present in the spectrum of the equal-time correlation matrix.

\section{Topological band insulator protected by two reflection symmetries in two dimensions}

\subsection{Hamiltonian and topological quantum numbers}

Our third example is defined by choosing $d=2$ and $N_{\text {orb }}=2$ in Eq. 2.1. We consider the two-dimensional plane $z=0$ from the three-dimensional Cartesian space $\mathbb{R}^{3}:=\{x \boldsymbol{x}+y \boldsymbol{y}+z \boldsymbol{z} \mid x, y, z \in \mathbb{R}\}$ with the orthonormal basis $\boldsymbol{x}, \boldsymbol{y}$, and $\boldsymbol{z}$. The singleparticle Hamiltonian is defined by (the convention $e>0$ is used for the electron charge)

$$
\hat{\mathcal{H}}:=\sum_{\sigma= \pm} \frac{\hbar^{2}}{2 m}\left[\hat{\boldsymbol{p}} \sigma_{0}-\frac{e}{c} \boldsymbol{A}(\hat{\boldsymbol{r}}) \sigma_{3}\right]^{2}
$$

where $\sigma_{0}$ for the $2 \times 2$ unit matrix and $\sigma$ for the Pauli matrices are the usual suspects,

$$
\hat{\boldsymbol{p}}:=\left(\begin{array}{c}
\hat{p}_{x} \\
\hat{p}_{y}
\end{array}\right)
$$

is the momentum operator in the plane $z=0$,

$$
\hat{\boldsymbol{r}}:=\left(\begin{array}{l}
\hat{r}_{x} \\
\hat{r}_{y}
\end{array}\right)
$$

is the position operator in the plane $z=0$, and $\boldsymbol{A}(\hat{r})$ is the classical electromagnetic vector potential such that

$$
(\boldsymbol{\nabla} \wedge \boldsymbol{A})(\boldsymbol{r})=B \boldsymbol{z}
$$

is the uniform magnetic of magnitude $B>0$ pointing along $\boldsymbol{z}$. This model describes an electron; with mass $m$, electric charge $-e$, and the conserved spin quantum number along the quantization axis defined by the eigenstates of the Pauli matrix $\sigma_{3}$; undergoing the spin quantum Hall effect generated by the matrix-valued field $B \sigma_{3} z$.

The eigenvalue spectrum consists of Landau levels with the single-particle energy eigenvalues

$$
\varepsilon_{n}=\hbar \omega_{\mathrm{c}}\left(n+\frac{1}{2}\right), \quad n=0,1,2, \cdots,
$$

where

$$
\omega_{\mathrm{c}}:=\frac{e B}{m c}
$$


is the cyclotron frequency. In addition to the usual orbital degeneracy $\Phi / \Phi_{0}$ of Landau levels with $\Phi$ the total magnetic flux and $\Phi_{0}:=h c / e$ the flux quantum, there is the Kramers' degeneracy under the composition of complex conjugation and conjugation by $\sigma_{2}$.

Each Landau level fully filled by $\Phi / \Phi_{0}$ electrons with spin up carries the Chern number $C_{+}=+1$. Each Landau level fully filled by $\Phi / \Phi_{0}$ electrons with spin down carries the Chern number $C_{-}=-1$. The contribution to the Hall conductivity from any completely filled Landau level is therefore

$$
\sigma_{\mathrm{QHE}}=\left(C_{+}+C_{-}\right) \frac{e^{2}}{h}=0 .
$$

The contribution to the spin Hall conductivity from any completely filled Landau level is

$$
\sigma_{\mathrm{SQHE}}=\frac{\hbar}{2 e}\left(C_{+}-C_{-}\right) \frac{e^{2}}{h}=\frac{e}{2 \pi} .
$$

On an infinite strip along the $\boldsymbol{x}$ direction of width $L_{y}$ along the $\boldsymbol{y}$ direction, we impose translation symmetry along the $\boldsymbol{y}$ direction through periodic boundary conditions. For this cylindrical geometry (recall Fig. 6 with the identification $i=y$ and $i+1=x$ ), it is convenient to choose the Landau gauge

$$
\boldsymbol{A}:=B\left(\begin{array}{l}
0 \\
x
\end{array}\right)
$$

for which normalized single-particle eigenstates $\left|n, \sigma, k_{y}\right\rangle$ in the lowest Landau level $n=0$ can be represented by the normalized eigen-wavefunctions in the lowest $(n=0)$ Landau levels

$$
\left\langle x, y \mid n=0, \sigma, k_{y}\right\rangle \equiv f_{k_{y}}^{(\sigma)}(x, y)=\frac{e^{+\mathrm{i} k_{y} y} e^{-\left(x-\sigma \ell_{B}^{2} k_{y}\right)^{2} /\left(2 \ell_{B}^{2}\right)}}{\left(\sqrt{\pi} \ell_{B} L_{y}\right)^{1 / 2}}
$$

for a spin $\sigma$ electron. Here, $\sigma= \pm$ is the spin of the electron along the quantization axis, $\boldsymbol{r}=(x, y) \in \mathbb{R}^{2}$ is the coordinate of the electron, and

$$
k_{y}=\frac{2 \pi}{L_{y}} m, \quad m=1, \cdots, \frac{\Phi}{\Phi_{0}},
$$

is the quantized wave number arising from the compactification along the width $L_{y}$ of the strip and

$$
\ell_{B}:=\sqrt{\frac{\hbar c}{e B}}
$$

is the magnetic length that we shall set to unity from now on.

\subsection{Symmetries}

The symmetries of the single-particle Hamiltonian defined by Eq. (6.1) are the following.

(i) Let $\mathscr{R}_{x}$ define the reflection about the horizontal axis $\boldsymbol{r}=(x, 0)$ by which the quantum numbers of the position and momentum operators transform according to

$$
\begin{aligned}
& \mathscr{R}_{x}: x \mapsto+x, \quad \mathscr{R}_{x}: y \mapsto-y, \\
& \mathscr{R}_{x}: k_{x} \mapsto+k_{x}, \quad \mathscr{R}_{x}: k_{y} \mapsto-k_{y},
\end{aligned}
$$


respectively. The symmetry

$$
\hat{\mathcal{O}}_{\mathscr{R}_{x}}^{\dagger} \hat{\mathcal{H}} \hat{\mathcal{O}}_{\mathscr{R}_{x}}=\hat{\mathcal{H}}
$$

then holds for the single-particle Hamiltonian 6.1a if $\mathscr{R}_{x}$ is represented by the composition of $x \mapsto+x, y \mapsto-y$ with the conjugation by either $\sigma_{x}$ or $\sigma_{y}$. We choose the representation

$$
\hat{\mathcal{O}}_{\mathscr{R}_{x}} \chi_{k_{y}}(x, y):=\sigma_{x} \chi_{-k_{y}}(x,-y)
$$

for any two-component spinor $\chi_{k_{y}}(x, y) \in \mathbb{C}^{2}$.

(ii) Let $\mathscr{R}_{y}$ define the reflection about the vertical axis $\boldsymbol{r}=(0, y)$ by which the quantum numbers of the position and momentum operators transform according to

$$
\begin{aligned}
& \mathscr{R}_{x}: x \mapsto-x, \quad \mathscr{R}_{x}: y \mapsto+y, \\
& \mathscr{R}_{x}: k_{x} \mapsto-k_{x}, \quad \mathscr{R}_{x}: k_{y} \mapsto+k_{y},
\end{aligned}
$$

respectively. The symmetry

$$
\hat{\mathcal{O}}_{\mathscr{R}_{y}}^{\dagger} \hat{\mathcal{H}} \hat{\mathcal{O}}_{\mathscr{R}_{y}}=\hat{\mathcal{H}}
$$

then holds for the single-particle Hamiltonian 6.1a) if $\mathscr{R}_{y}$ is represented by the composition of $x \mapsto-x, y \mapsto+y$ with the conjugation by $\sigma_{x}$, given the choice made in Eq. $6.5 \mathrm{c}$, i.e.,

$$
\hat{\mathcal{O}}_{\mathscr{R}_{y}} \chi_{k_{y}}(x, y):=\sigma_{x} \chi_{k_{y}}(-x, y)
$$

for any two-component spinor $\chi_{k_{y}}(x, y) \in \mathbb{C}^{2}$. With this choice, the commutation relation $\left[\mathscr{R}_{x}, \mathscr{R}_{y}\right]=0$ is faithfully represented, i.e.,

$$
\left[\hat{\mathcal{O}}_{\mathscr{R}_{x}}, \hat{\mathcal{O}}_{\mathscr{R}_{y}}\right]=0 .
$$

(iii) Let $\mathscr{T}$ define reversal of time by which time and the quantum numbers of the position and momentum operators transform according to

$$
\begin{aligned}
& \mathscr{T}: t \mapsto-t, \\
& \mathscr{R}_{x}: x \mapsto x, \quad \mathscr{R}_{x}: y \mapsto y, \\
& \mathscr{R}_{x}: k_{x} \mapsto-k_{x}, \quad \mathscr{R}_{x}: k_{y} \mapsto-k_{y},
\end{aligned}
$$

respectively. The symmetry

$$
\hat{\mathcal{O}}_{\mathscr{T}}^{\dagger} \hat{\mathcal{H}} \hat{\mathcal{O}}_{\mathscr{T}}=\hat{\mathcal{H}}
$$

then holds for the single-particle Hamiltonian 6.1a) if $\mathscr{T}$ is represented by the composition of charge conjugation with the conjugation by $\sigma_{x}$, i.e.,

$$
\hat{\mathcal{O}}_{\mathscr{T}} \chi_{k_{y}}(x, y):=\sigma_{x} \chi_{-k_{y}}^{*}(x, y)
$$

for any two-component spinor $\chi_{k_{y}}(x, y) \in \mathbb{C}^{2}$. With this choice, the commutation relations $\left[\mathscr{R}_{x}, \mathscr{T}\right]=\left[\mathscr{R}_{y}, \mathscr{T}\right]=0$ are faithfully represented, i.e.,

$$
\left[\hat{\mathcal{O}}_{\mathscr{T}}, \hat{\mathcal{O}}_{\mathscr{R}_{x}}\right]=\left[\hat{\mathcal{O}}_{\mathscr{T}}, \hat{\mathcal{O}}_{\mathscr{R}_{y}}\right]=0
$$

Observe that had we implemented the two reflections by choosing $\sigma_{y}$ instead of $\sigma_{x}$ on the right-hand sides of Eqs. (6.5c) and 6 6.6c), then the transformation of reversal of time would anti-commute with both reflections. 


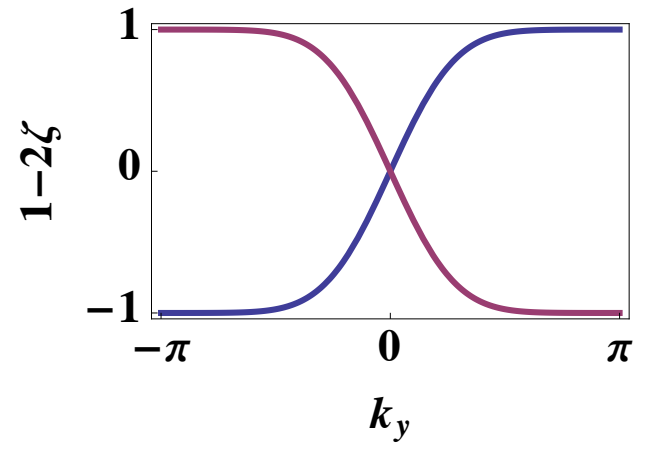

Figure 8. (Color online) The entanglement spectrum $\sigma\left(\hat{Q}_{k_{y} A}\right)$ with $\hat{Q}_{k_{y} A}$ defined by Eqs. 6.16 and 6.17) for the spin quantum Hall effect. There are two branches of eigenstates with opposite chiralities (these chiralities are denoted by the colors blue and red) that cross at vanishing energy and momentum.

\subsection{Partition and zero modes}

The counterpart to the projection of $\hat{\psi}_{I}^{\dagger} \hat{\psi}_{J}$ in the equal-time one-point correlation matrix 2.5 onto the completely filled Landau level $n=0$ is the operator

$$
\hat{\mathcal{C}}=\sum_{\sigma= \pm} \sum_{L_{y}}^{\Phi / \Phi_{0}}\left|n=0, \sigma, k_{y}\right\rangle\left\langle n=0, \sigma, k_{y}\right|
$$

with the matrix element

$$
\mathcal{C}_{\sigma, k_{y}}\left(x, x^{\prime}\right) \equiv\left\langle n=0, \sigma, k_{y}, x|\hat{\mathcal{C}}| n=0, \sigma, k_{y}, x^{\prime}\right\rangle=\frac{1}{\sqrt{\pi}} e^{-\frac{1}{2}\left[\left(x-\sigma k_{y}\right)^{2}+\left(x^{\prime}-\sigma k_{y}\right)^{2}\right]}
$$

for any $x, x^{\prime} \in \mathbb{R}$. One verifies that the $2 \times 2$ matrix

$$
\hat{\mathcal{C}}_{k_{y}}\left(x, x^{\prime}\right):=\mathcal{C}_{+, k_{y}}\left(x, x^{\prime}\right) \sigma_{11}+\mathcal{C}_{-, k_{y}}\left(x, x^{\prime}\right) \sigma_{22},
$$

where $\sigma_{11}:=\left(\sigma_{0}+\sigma_{3}\right) / 2$ and $\sigma_{22}:=\left(\sigma_{0}-\sigma_{3}\right) / 2$, obeys

$$
\begin{aligned}
& \hat{\mathcal{O}}_{\mathscr{R}_{x}}^{\dagger} \hat{\mathcal{C}}_{-k_{y}}\left(x, x^{\prime}\right) \hat{\mathcal{O}}_{\mathscr{R}_{x}}=\hat{\mathcal{C}}_{k_{y}}\left(x, x^{\prime}\right), \\
& \hat{\mathcal{O}}_{\mathscr{R}_{y}}^{\dagger} \hat{\mathcal{C}}_{k_{y}}\left(-x,-x^{\prime}\right) \hat{\mathcal{O}}_{\mathscr{R}_{y}}=\hat{\mathcal{C}}_{k_{y}}\left(x, x^{\prime}\right), \\
& \hat{\mathcal{O}}_{\mathscr{T}}^{\dagger} \hat{\mathcal{C}}_{-k_{y}}\left(x, x^{\prime}\right) \hat{\mathcal{O}}_{\mathscr{T}}=\hat{\mathcal{C}}_{k_{y}}\left(x, x^{\prime}\right) .
\end{aligned}
$$

We define the partition

$$
A:=\left\{\sigma, k_{y}, x \mid \sigma= \pm, k_{y}=\frac{2 \pi}{L_{y}}, \cdots, \frac{2 \pi \Phi}{L_{y} \Phi_{0}}, x<0\right\}
$$

and

$$
B:=\left\{\sigma, k_{y}, x \mid \sigma= \pm, k_{y}=\frac{2 \pi}{L_{y}}, \cdots, \frac{2 \pi \Phi}{L_{y} \Phi_{0}}, x>0\right\}
$$

for the single-particle labels. The restriction of the equal-time one-point correlation operator defined by Eq. 6.10 to $x<0$ and $x^{\prime}<0$ defines the operator $\hat{C}_{A}$ with its matrix elements 
$C_{\sigma, k_{y} A}\left(x, x^{\prime}\right)$ and the $2 \times 2$ matrix $\hat{C}_{k_{y} A}\left(x, x^{\prime}\right)$. The restriction of the equal-time one-point correlation operator defined by Eq. 6.10 to $x>0$ and $x^{\prime}>0$ defines the operator $\hat{C}_{B}$ with its matrix elements $C_{\sigma, k_{y} B}\left(x, x^{\prime}\right)$ and the $2 \times 2$ matrix $\hat{C}_{k_{y} B}\left(x, x^{\prime}\right)$. With this partition, for any $x, x^{\prime}<0$,

$$
\begin{aligned}
& \hat{\mathcal{O}}_{\mathscr{R}_{x}}^{\dagger} \hat{C}_{-k_{y} A}\left(x, x^{\prime}\right) \hat{\mathcal{O}}_{\mathscr{R}_{x}}=\hat{C}_{k_{y} A}\left(x, x^{\prime}\right), \\
& \hat{\mathcal{O}}_{\mathscr{R}_{y}}^{\dagger} \hat{C}_{k_{y} A}\left(x, x^{\prime}\right) \hat{\mathcal{O}}_{\mathscr{R}_{y}}=\hat{C}_{k_{y} B}\left(-x,-x^{\prime}\right), \\
& \hat{\mathcal{O}}_{\mathscr{T}}^{\dagger} \hat{C}_{-k_{y} A}\left(x, x^{\prime}\right) \hat{\mathcal{O}}_{\mathscr{T}}=\hat{C}_{k_{y} A}\left(x, x^{\prime}\right) .
\end{aligned}
$$

The same equations hold if we do $x \rightarrow-x, x^{\prime} \rightarrow-x^{\prime}, A \rightarrow B$, and $B \rightarrow A$. The restriction of the equal-time one-point correlation operator defined by Eq. 6.10) to $x<0$ and $x^{\prime}>0$ defines the operator $\hat{C}_{A B}$ with its matrix elements $C_{\sigma, k_{y} A B}\left(x, x^{\prime}\right)$ and the $2 \times 2$ matrix $\hat{C}_{k_{y} A B}\left(x, x^{\prime}\right)$. Finally, the restriction of the equal-time one-point correlation operator defined by Eq. 6.10) to $x>0$ and $x^{\prime}<0$ defines the operator $\hat{C}_{B A}=\hat{C}_{A B}^{\dagger}$ with its matrix elements $C_{\sigma, k_{y} B A}\left(x, x^{\prime}\right)=C_{\sigma, k_{y} A B}^{\dagger}\left(x^{\prime}, x\right)$ and the $2 \times 2$ matrix $\hat{C}_{k_{y} B A}\left(x, x^{\prime}\right)=\hat{C}_{k_{y} A B}^{\dagger}\left(x^{\prime}, x\right)$.

The normalizable eigenstates of $\hat{C}_{k_{y} A}$ are represented by

$$
\phi_{+, k_{y}}(x)=\frac{1}{\sqrt{2} \pi^{1 / 4}}\left(\begin{array}{c}
e^{-\frac{1}{2}\left(x-k_{y}\right)^{2}} \\
0
\end{array}\right)
$$

and

$$
\phi_{-, k_{y}}(x)=\frac{1}{\sqrt{2} \pi^{1 / 4}}\left(\begin{array}{c}
0 \\
e^{-\frac{1}{2}\left(x+k_{y}\right)^{2}}
\end{array}\right)
$$

for any $-\infty<x \leq 0$. In the thermodynamic limit $L_{y} \rightarrow \infty$ holding the electron density fixed, the corresponding eigenvalues are given by

$$
\zeta_{ \pm}\left(k_{y}\right)=\frac{1}{\sqrt{\pi}} \int_{-\infty}^{0} \mathrm{~d} x e^{-\left(x \mp k_{y}\right)^{2}}=: \frac{1}{2}\left[1-\operatorname{erf}\left( \pm k_{y}\right)\right] .
$$

The spectrum $1-2 \zeta_{ \pm}\left(k_{y}\right)$ of

$$
\hat{Q}_{k_{y} A}:=\hat{\mathbb{I}}-2 \hat{C}_{k_{y} A}
$$

is shown in Fig. 8 Two chiral edge modes of opposite chirality are seen crossing the spectrum with a crossing at vanishing entanglement eigenvalue.

On the one hand, Eqs. 6.13a and 6.13c) imply that the reflection about the horizontal axis 6.5 and reversal of time 6.8 are represented by the operators $\hat{\mathcal{O}}_{\mathscr{R}_{x}}$ and $\hat{\mathcal{O}}_{\mathscr{T}}$, respectively, that are block diagonal with respect to the partition of the single-particle labels into the sets $A$ and $B$ defined by Eq. (6.12). As explained in Eqs. (2.37) and (2.38), the upper-left block 6.17) inherits these symmetries from $\hat{\mathcal{H}}$ defined in Eq. (6.1).

On the one hand, Eq. $6.13 \mathrm{~b}$ implies that $\hat{\mathcal{O}}_{\mathscr{R}_{y}}$ is block off diagonal with respect to the partition of the single-particle labels into the sets $A$ and $B$ defined by Eq. (6.12). The 
counterparts to Eqs. 2.39) and 2.40) then hold, so that we may apply the counterpart to the spectral symmetry 2.45 ).

To proceed, for any $x, x^{\prime}<0$ and $k_{y}, k_{y}^{\prime}=2 \pi / L_{y}, \cdots,(2 \pi \Phi) /\left(L_{y} \Phi_{0}\right)$, we make use of the representations

$$
\hat{O}_{k_{y}, k_{y}^{\prime} \mathscr{R}_{x} A}\left(x, x^{\prime}\right):=\left\langle k_{y}, x\left|\hat{\mathcal{O}}_{\mathscr{R}_{x}}\right| k_{y}^{\prime}, x^{\prime}\right\rangle=\delta_{k_{y}+k_{y}^{\prime}, 0} \delta\left(x-x^{\prime}\right) \sigma_{x},
$$

for the reflection about the horizontal axis,

$$
\hat{O}_{k_{y}, k_{y}^{\prime} \mathscr{R}_{y} A}\left(x, x^{\prime}\right):=\left\langle k_{y}, x\left|\hat{\mathcal{O}}_{\mathscr{R}_{y}}\right| k_{y}^{\prime}, x^{\prime}\right\rangle=\delta_{k_{y}, k_{y}^{\prime}} \delta\left(x+x^{\prime}\right) \sigma_{x}
$$

for the reflection about the vertical axis, and

$$
\hat{O}_{k_{y}, k_{y}^{\prime} \mathscr{T} A}\left(x, x^{\prime}\right):=\left\langle k_{y}, x\left|\hat{\mathcal{O}}_{\mathscr{T}}\right| k_{y}^{\prime}, x^{\prime}\right\rangle=\delta_{k_{y}+k_{y}^{\prime}, 0} \delta\left(x-x^{\prime}\right) \sigma_{y} K,
$$

for the reversal of time ( $K$ denotes complex conjugation). Consequently, the upper-left block (6.17) obeys the symmetries

$$
\begin{aligned}
\hat{Q}_{k_{y} A}\left(x, x^{\prime}\right)= & \sum_{k_{y}^{\prime \prime}} \sum_{k_{y}^{\prime \prime \prime}} \int_{-\infty}^{0} \mathrm{~d} x^{\prime \prime} \int_{-\infty}^{0} \mathrm{~d} x^{\prime \prime \prime} \\
& \hat{O}_{k_{y}, k_{y}^{\prime \prime} \mathscr{R}_{x} A}\left(x, x^{\prime \prime}\right) \delta_{k_{y}^{\prime \prime}, k_{y}^{\prime \prime \prime}} \hat{Q}_{k_{y}^{\prime \prime \prime} A}\left(x^{\prime \prime}, x^{\prime \prime \prime}\right) \hat{O}_{k_{y}^{\prime \prime \prime}, k_{y}^{\prime} \mathscr{R}_{x} A}\left(x^{\prime \prime \prime}, x^{\prime}\right) \\
= & \sigma_{x} \hat{Q}_{-k_{y} A}\left(x, x^{\prime}\right) \sigma_{x}
\end{aligned}
$$

under reflection about the horizontal axis and

$$
\begin{aligned}
\hat{Q}_{k_{y} A}\left(x, x^{\prime}\right)= & \sum_{k_{y}^{\prime \prime}} \sum_{k_{y}^{\prime \prime \prime}} \int_{-\infty}^{0} \mathrm{~d} x^{\prime \prime} \int_{-\infty}^{0} \mathrm{~d} x^{\prime \prime \prime} \\
& \hat{O}_{k_{y}, k_{y}^{\prime \prime} \mathscr{T} A}\left(x, x^{\prime \prime}\right) \delta_{k_{y}^{\prime \prime}, k_{y}^{\prime \prime \prime}} \hat{Q}_{k_{y}^{\prime \prime \prime} A}\left(x^{\prime \prime}, x^{\prime \prime \prime}\right) \hat{O}_{k_{y}^{\prime \prime \prime}, k_{y}^{\prime} \mathscr{T} A}\left(x^{\prime \prime \prime}, x^{\prime}\right) \\
= & \sigma_{y} \hat{Q}_{-k_{y} A}^{*}\left(x, x^{\prime}\right) \sigma_{y}
\end{aligned}
$$

under reversal of time. The symmetry of the Hamiltonian 6.1a under reflection about the vertical axis is turned into the spectral symmetry

$$
\begin{aligned}
-\hat{Q}_{k_{y} A}\left(x, x^{\prime}\right)= & \sum_{k_{y}^{\prime \prime}} \sum_{k_{y}^{\prime \prime \prime}} \int_{-\infty}^{0} \mathrm{~d} x^{\prime \prime} \int_{-\infty}^{0} \mathrm{~d} x^{\prime \prime \prime} \\
& \hat{\Gamma}_{k_{y}, k_{y}^{\prime \prime} \mathscr{R}_{y} A}\left(x, x^{\prime \prime}\right) \delta_{k_{y}^{\prime \prime}, k_{y}^{\prime \prime \prime}} \hat{Q}_{k_{y}^{\prime \prime \prime} A}\left(x^{\prime \prime}, x^{\prime \prime \prime}\right) \hat{\Gamma}_{k_{y}^{\prime \prime}, k_{y}^{\prime} \mathscr{R}_{y} A}\left(x^{\prime \prime \prime}, x^{\prime}\right),
\end{aligned}
$$

where

$$
\begin{aligned}
\hat{\Gamma}_{k_{y}, k_{y}^{\prime} \mathscr{R}_{y} A}\left(x, x^{\prime}\right) & =\sum_{k_{y}^{\prime \prime}} \int_{0}^{+\infty} \mathrm{d} x^{\prime \prime} \delta_{k_{y}, k_{y}^{\prime \prime}} \hat{C}_{k_{y} A B}\left(x, x^{\prime \prime}\right) \hat{O}_{k_{y}^{\prime \prime}, k_{y}^{\prime} \mathscr{R}_{y} A}^{\dagger}\left(x^{\prime \prime}, x^{\prime}\right) \\
& =\delta_{k_{y}, k_{y}^{\prime}} \frac{1}{\sqrt{\pi}}\left[e^{-\frac{1}{2}\left[\left(x-k_{y}\right)^{2}+\left(x^{\prime}+k_{y}\right)^{2}\right]} \sigma_{12}+e^{-\frac{1}{2}\left[\left(x+k_{y}\right)^{2}+\left(x^{\prime}-k_{y}\right)^{2}\right]} \sigma_{21}\right]
\end{aligned}
$$


with $\sigma_{12}:=\left(\sigma_{1}+\mathrm{i} \sigma_{2}\right) / 2$ and $\sigma_{21}:=\left(\sigma_{1}-\mathrm{i} \sigma_{2}\right) / 2$. For any $x<0$ and $k_{y}=$ $2 \pi / L_{y}, \cdots,(2 \pi \Phi) /\left(L_{y} \Phi_{0}\right)$, one verifies the eigenvalue equation

$$
\sum_{k_{y}^{\prime}} \int_{-\infty}^{0} \mathrm{~d} x^{\prime} \hat{\Gamma}_{k_{y}, k_{y}^{\prime} \mathscr{R}_{y} A}\left(x, x^{\prime}\right) \phi_{ \pm, k_{y}^{\prime}}\left(x^{\prime}\right)=\frac{1}{2}\left[1-\operatorname{erf}\left(k_{y}\right)\right] \phi_{\mp, k_{y}}(x) .
$$

\subsection{Stability analysis of the zero modes}

We are after the stability of the crossings at vanishing energy and momentum that characterizes the edge states in the Hamiltonian defined by Eq. (6.1) for a cylindrical geometry and the states in the entanglement spectra localized on the entangling boundary at $x=0$ defined in Sec.6.3. We are going to show that the reflection symmetries $\mathscr{R}_{x}$ and $\mathscr{R}_{y}$ do not protect the edge states on the physical boundaries, but they protect the edge states on the entangling boundary.

6.4.1. Hamiltonian spectrum If we linearize the edge state spectrum close to vanishing energy and momentum, we get the effective Hamiltonian (the group velocity has been set to one)

$$
\hat{\mathcal{H}}_{\text {edges }}\left(k_{y}\right) \approx k_{y} Y_{33}
$$

in the cylindrical geometry of Fig. $6(i=y$ and $i+1=x)$, where we have introduced a second set of $2 \times 2$ matrices generated by the unit $2 \times 2$ matrix $\rho_{0}$ an the Pauli matrices $\rho_{1}$, $\rho_{2}$, and $\rho_{3}$ when defining

$$
Y_{\mu \nu}:=\sigma_{\mu} \otimes \rho_{\nu}
$$

for $\mu, \nu=0,1,2,3$. The matrices $\rho_{0}, \rho_{1}, \rho_{2}$, and $\rho_{3}$ encode the mixing of edge states localized on opposite edges of the cylinder. Reflection about the $x$ axis, reflection about the $y$ axis, and reversal of time are represented by

$$
\begin{aligned}
& \hat{\mathcal{O}}_{\text {edges } \mathscr{R}_{x}}\left(k_{y}, k_{y}^{\prime}\right):=\delta\left(k_{y}+k_{y}^{\prime}\right) Y_{10}, \\
& \hat{\mathcal{O}}_{\text {edges } \mathscr{R}_{y}}\left(k_{y}, k_{y}^{\prime}\right):=\delta\left(k_{y}-k_{y}^{\prime}\right) Y_{11}, \\
& \hat{\mathcal{O}}_{\text {edges } \mathscr{T}}\left(k_{y}, k_{y}^{\prime}\right):=\delta\left(k_{y}+k_{y}^{\prime}\right) Y_{20} K,
\end{aligned}
$$

where $K$ denotes charge conjugation. In a gradient expansion, any perturbation of the form

$$
\hat{\mathcal{V}}_{\text {edges } \mu \nu}=v_{\mu \nu} Y_{\mu \nu}
$$

for some energy scale $v_{\mu \nu} \in \mathbb{R}$ that is smaller than the cyclotron energy, commutes with the three transformations 6.26 , and anti-commutes with $Y_{33}$, say $\hat{\mathcal{V}}_{\text {edges } 01}$, opens a spectral gap on the edges.

In the thermodynamic limit by which the length of the cylinder is taken to infinity keeping the density of electrons fixed, there is no mixing between edge states localized on opposite boundaries of the cylinder. The effective Hamiltonian on a single edge becomes

$$
\hat{\mathcal{H}}_{\text {edge }}\left(k_{y}\right) \approx k_{y} \sigma_{3} .
$$


It commutes with

$$
\begin{aligned}
& \hat{\mathcal{O}}_{\text {edge } \mathscr{R}_{x}}\left(k_{y}, k_{y}^{\prime}\right):=\delta\left(k_{y}+k_{y}^{\prime}\right) \sigma_{1}, \\
& \hat{\mathcal{O}}_{\text {edge } \mathscr{T}}\left(k_{y}, k_{y}^{\prime}\right):=\delta\left(k_{y}+k_{y}^{\prime}\right) \sigma_{2} K,
\end{aligned}
$$

where $K$ denotes charge conjugation. In a gradient expansion, a generic perturbation on this single edge is of the form

$$
\hat{\mathcal{V}}_{\text {edge }}=\sum_{\mu=0}^{3} v_{\mu} \sigma_{\mu}
$$

with any $v_{\mu} \in \mathbb{R}$ much smaller than the bulk gap.

Imposing reflection symmetry enforces the conditions $v_{2}=v_{3}=0$ on the perturbation $\hat{\mathcal{V}}_{\text {edge }}$, i.e., a reflection symmetric perturbation can open a gap at the crossing in the unperturbed spectrum. The crossing in the unperturbed spectrum of a single edge is thus not protected by having two reflection symmetries in the bulk Hamiltonian. This is not true anymore for the entanglement spectrum.

Imposing time-reversal symmetry enforces the conditions $v_{1}=v_{2}=v_{3}=0$ on the perturbation $\hat{\mathcal{V}}_{\text {edge }}$, i.e., a time-reversal symmetric perturbation does not destroy the crossing, it merely shifts the crossing in the unperturbed spectrum at vanishing momentum to a nonvanishing energy.

6.4.2. Entanglement spectrum In the thermodynamic limit by which the length of the cylinder is taken to infinity keeping the density of electrons fixed, there is no mixing between states localized on the physical boundaries at $x= \pm \infty$ and the entangling boundary at $x=0$. The entangling edge states close to the crossing at vanishing entangling eigenvalue and momentum of Fig. 8 are governed by the effective Hamiltonian (the group velocity has been set to one)

$$
\hat{\mathcal{Q}}_{\text {edge } A}\left(k_{y}\right) \approx k_{y} \sigma_{3} .
$$

The representations of the two symmetry transformations 6.29a and 6.29b remain valid. In addition, the spectral symmetry transformation (6.22) takes the form of an anti-commutation with

$$
\hat{\Gamma}_{\text {edge } \mathscr{R}_{y} A}\left(k_{y}, k_{y}^{\prime}\right) \propto \delta\left(k_{y}-k_{y}^{\prime}\right) \sigma_{1}
$$

when $x=x^{\prime}=0$ and $k_{y}, k_{y}^{\prime} \approx 0$. A perturbation of the form 6.30 is then restricted to the conditions $v_{2}=v_{3}=0$ if it commutes with the generator $6.29 \mathrm{a}$ for reflection about the horizontal axis. A perturbation of the form 6.30 is restricted to the conditions $v_{0}=v_{1}=0$ if it anti-commutes with the generator 6.32) for reflection about the vertical axis. Imposing both reflection symmetries on the Hamiltonian thus protects the crossing at vanishing entangling eigenvalue and momentum in Fig. 8from any perturbation, unlike for the crossing at vanishing energy and momentum of the edge states for the Hamiltonian.

Finally, a perturbation of the form 6.30 is restricted to the conditions $v_{1}=v_{2}=$ $v_{3}=0$ if it commutes with the generator $(6.29 \mathrm{~b})$ for reversal of time. Imposing timereversal symmetry on the Hamiltonian thus protects the existence of a crossing at vanishing momentum in Fig. 8 from any perturbation. 


\section{Graphene with Kekule order as an inversion-symmetric topological insulator in two dimensions}

\subsection{Introduction}

Graphene has two single-particle bands that touch linearly at two inequivalent points $\boldsymbol{K}$ and $\boldsymbol{K}^{\prime}$ from the first Brillouin zone of the triangular lattice (BZT). These two inequivalent points from the BZT of graphene are called Dirac points. If graphene is modeled by a tightbinding model with two bands, whereby spinless electrons can only hop with a uniform amplitude $t$ between the nearest-neighbor sites of the honeycomb lattice, then the linear band touching is located at the two inequivalent corners of the hexagonal BZT of graphene. [42, 43] Graphene is a planar semi-metal with a low-energy and long-wave length electronic structure that can be modeled by a $4 \times 4$ massless Dirac Hamiltonian in $(2+1)$-dimensional space and time. The rank four of the Dirac matrices entering this Dirac Hamiltonian arises because only the two bands with a linear touching are kept in the low-energy sector of graphene and after linearization of the single-particle spectrum about the two Dirac points $\boldsymbol{K}$ and $\boldsymbol{K}^{\prime}$.

The Kekule distortion is a pattern of symmetry breaking on the honeycomb lattice by which the nearest-neighbor hopping amplitude takes the two distinct real values $t_{1}$ and $t_{2}$, respectively. This pattern of symmetry breaking is depicted in Fig. 9(a) through the coloring of the nearest-neighbor bonds of the honeycomb lattice. The Kekule distortion is weak if $\left|t_{1}-t_{2}\right| \ll\left|t_{1}+t_{2}\right| / 2$, in which case a single-particle gap $\Delta_{\mathrm{K}} \propto\left|t_{1}-t_{2}\right|$ that is much smaller than the band width $\left(\propto\left|t_{1}+t_{2}\right| / 2\right)$ opens up a the Dirac points. A Kekule distortion can be induced by fine-tuning of sufficiently large repulsive interactions. [28, 44] A Kekule distortion is also favored in the presence of a sufficiently large magnetic field by some phonons. [45, 46] A Kekule distortion may also arise locally at the core of a vortex in the (proximity-induced) superconducting phase of graphene. [47] Finally, a Kekule distortion has been observed in artificial graphene (molecular graphene) obtained by patterning carbon monoxide molecules on the 111 surface of copper.[48]

A distinctive feature of graphene is a density of states that vanishes linearly if singleparticle energies are measured relative to the energy of the Dirac points at sufficiently small energies. This semi-metallic behavior is turned into a semiconducting one if a Kekule distortion opens a gap at the Dirac points that is larger than the chemical potential measured relative to the energy at the Dirac points. Graphene with a Kekule distortion is thus a band insulator. From the point of view of the ten-fold classification of band insulators in twodimensional space, [36, 37, 38, 39, 40, 41] graphene with a Kekule distortion is topologically trivial in that it does not support gapless edge states in an open geometry that are robust to the breaking of translation invariance by an on-site real-valued potential, say. Nevertheless, graphene with a Kekule distortion supports unusual quantum numbers if the Kekule distortion is defective. For example, a point defect in the Kekule distortion binds locally a fractional value of the electron charge. [28, 46, 49, 50]

The goal of Sec. 7 is to study the entanglement spectrum of graphene with a Kekule distortion and show that graphene with a Kekule distortion is another example of a symmetry 


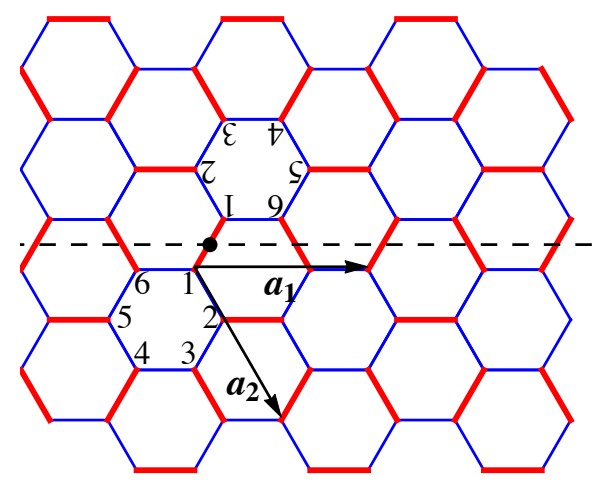

(a)

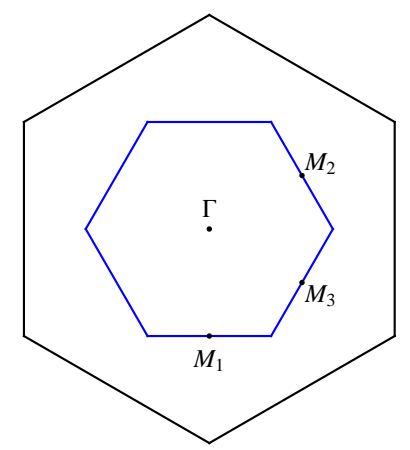

(b)

Figure 9. (Color online) (a) The simplest tight-binding model for graphene with a Kekule distortion is defined by allowing spinless electrons to hop between any two nearest-neighbor sites of the honeycomb lattice with the real-valued modulated amplitudes $t_{1}$ and $t_{2}$ if a nearestneighbor bond is colored in red or blue, respectively. The repeat unit cell of the strong and weak bonds associated to the Kekule distortion can be chosen to be made of the following three hexagons below the dashed line. The first hexagon is colored in blue and has six vertices numbered clockwise from 1 to 6 . The second hexagon shares the blue bond $\langle 12\rangle$ with the first one. The third hexagon shares the blue bond $\langle 23\rangle$ to the first one. This unit cell comprised of these three hexagons is three time as large as the repeat unit cell of the honeycomb lattice with all nearest-neighbor bonds colored in black (the limiting case when $t_{1}=t_{2}$ ). This enlarged repeat unit cell has 6 inequivalent sites. The spanning vectors of the honeycomb lattice with the Kekule coloring of nearest-neighbor bonds are $\boldsymbol{a}_{1}$ and $\boldsymbol{a}_{2}$. (b) The large hexagon colored in black defines the first Brillouin zone of the triangular lattice. The small hexagon colored in blue defines the first Brillouin zone of the honeycomb lattice with the Kekule coloring of nearest-neighbor bonds. The ratio of the area of the black hexagon to the area of the blue hexagon is three to one. If the honeycomb lattice is cut along the horizontal dashed line, an armchair edge is obtained. The point denoted by $\bullet$ at the mid-point where the dashed line intersects the nearest-neighbor bond coming out of vertex 1 below the dashed line defines the inversion center. The points $\Gamma, M_{1}, M_{2}$ and $M_{3}$ from the first Brillouin zone of the honeycomb lattice with the Kekule coloring of nearest-neighbor bonds are invariant (fixed) under this inversion.

protected topological phase of matter.

\subsection{Hamiltonian}

We start from a honeycomb lattice $\Lambda$, whose sites are denoted with the symbol $i$. We then color the nearest-neighbor bonds of $\Lambda$ with two colors, say red and blue, as is done in Fig. 9(a), A pair of nearest-neighbor sites of the honeycomb lattice is denoted by $\left\langle\boldsymbol{i}_{1} \boldsymbol{j}_{1}\right\rangle\left(\left\langle\boldsymbol{i}_{2} \boldsymbol{j}_{2}\right\rangle\right)$ if they are covered by a bond colored in red (blue) in Fig. 9(a).

To each site $\boldsymbol{i}$, we assign the anti-commuting pair $\hat{c}_{\boldsymbol{i}}^{\dagger}$ and $\hat{c}_{\boldsymbol{i}}$ of creation and annihilation operators, respectively. We then model graphene with a Kekule distortion on which spinless fermions hop by the Hamiltonian

$$
\hat{H}_{\mathrm{K}}:=\sum_{n=1,2} t_{n} \sum_{\left\langle\boldsymbol{i}_{n} \boldsymbol{j}_{n}\right\rangle}\left(\hat{c}_{\boldsymbol{i}_{n}}^{\dagger} \hat{c}_{\boldsymbol{j}_{n}}+\text { H.c. }\right),
$$


where

$$
t_{n}:=-t+\frac{2 \cos \left((n-1) \frac{2 \pi}{3}\right)}{3} \Delta_{\mathrm{K}}
$$

with $t$ and $\Delta_{\mathrm{K}}$ both real-valued. The gapless spectrum of graphene with the uniform hopping amplitude $t$ is recovered when $\Delta_{\mathrm{K}}=0$.

The energy scale $\left|\Delta_{K}\right| \ll|t|$ breaks the point-group symmetry of the honeycomb lattice $\Lambda$ as is implied by the colors blue and red of inequivalent nearest-neighbor bonds in Fig. 9(a). The repeat unit cell of the honeycomb lattice $\Lambda$ decorated by weak and strong bonds as is implied by the colorings in Fig. 9(a) can be chosen to be the three hexagons sharing pairwise the blue bond $\langle 12\rangle$ and the blue bond $\langle 23\rangle$ in Fig. 9(a). The corresponding spanning vectors of the honeycomb lattice are then $\boldsymbol{a}_{1}$ and $\boldsymbol{a}_{2}$. We use the convention whereby we label the 6 sites of the blue hexagon as is done below the dashed line in Fig. 9(a). The site 2 belongs to one repeat unit cell. The sites 1, 3, 5 and their three images by translations belong to two repeat unit cells. The sites 4 and 6 and their four images by translations belong to three repeat unit cells. Hence, there are a total of $1+3+2=6$ inequivalent sites in the repeat unit cell for the honeycomb lattice with the Kekule distortion. We label the site 2 from the repeat unit cell for the honeycomb lattice with the Kekule distortion by $\boldsymbol{I}$. We may then introduce the spinor

$$
\hat{\psi}_{\boldsymbol{I}}^{\dagger}:=\left(\begin{array}{llllll}
\hat{\psi}_{\mathbf{I} 1}^{\dagger} & \hat{\psi}_{\mathbf{I} 2}^{\dagger} & \hat{\psi}_{\mathbf{I} 3}^{\dagger} & \hat{\psi}_{\boldsymbol{I} 4}^{\dagger} & \hat{\psi}_{\mathbf{I} 5}^{\dagger} & \hat{\psi}_{\mathbf{I} 6}^{\dagger}
\end{array}\right)
$$

obeying the fermion algebra

$$
\left\{\hat{\psi}_{\boldsymbol{I} a}, \hat{\psi}_{\boldsymbol{I}^{\prime} a^{\prime}}^{\dagger}\right\}=\delta_{\boldsymbol{I}, \boldsymbol{I}^{\prime}} \delta_{a, a^{\prime}}, \quad\left\{\hat{\psi}_{\boldsymbol{I} a}^{\dagger}, \hat{\psi}_{\boldsymbol{I}^{\prime} a^{\prime}}^{\dagger}\right\}=0,
$$

and whose components create a spinless fermions on any one of the six inequivalent sites from the repeat unit cell $\boldsymbol{I}$ for the honeycomb lattice with the Kekule distortion. By performing a Fourier transformation to reciprocal space with momenta $k$ restricted to one third of the first Brillouin zone of graphene, we may rewrite Hamiltonian (7.1) as

$$
\hat{H}_{\mathrm{K}}=\sum_{\boldsymbol{k} \in \mathrm{BZK}} \hat{\psi}_{\boldsymbol{k}}^{\dagger} \mathcal{H}_{\boldsymbol{k}} \hat{\psi}_{\boldsymbol{k}}
$$

where the $6 \times 6$ Hermitian matrix $\mathcal{H}_{\boldsymbol{k}}$ is given by

$$
\mathcal{H}_{\boldsymbol{k}}=\left(\begin{array}{cccccc}
0 & t_{2} & 0 & t_{1} e^{-\mathrm{i}\left(k_{2}-k_{1}\right)} & 0 & t_{2} \\
t_{2} & 0 & t_{2} & 0 & t_{1} e^{+\mathrm{i} k_{1}} & 0 \\
0 & t_{2} & 0 & t_{2} & 0 & t_{1} e^{+\mathrm{i} k_{2}} \\
t_{1} e^{+\mathrm{i}\left(k_{2}-k_{1}\right)} & 0 & t_{2} & 0 & t_{2} & 0 \\
0 & t_{1} e^{-\mathrm{i} k_{1}} & 0 & t_{2} & 0 & t_{2} \\
t_{2} & 0 & t_{1} e^{-\mathrm{i} k_{2}} & 0 & t_{2} & 0
\end{array}\right) .
$$

The acronym BZK stands for the reduced Brillouin zone in Fig. 9(b) associated to the repeat unit cell of the honeycomb lattice with a Kekule distortion. It covers one third of the area of the BZT. 


\subsection{Symmetries}

The symmetry

$$
\mathcal{H}_{-\boldsymbol{k}}^{*}=\mathcal{H}_{+\boldsymbol{k}}
$$

implements time-reversal symmetry for spinless fermions.

The spectral symmetry

$$
\mathcal{S}^{-1} \mathcal{H}_{k} \mathcal{S}=-\mathcal{H}_{k}
$$

where

$$
\mathcal{S}:=\operatorname{diag}(1,-1,1,-1,1,-1)
$$

implements the chiral (sublattice) spectral symmetry.

The symmetry

$$
\mathcal{P}^{-1} \mathcal{H}_{-k} \mathcal{P}=\mathcal{H}_{k}
$$

where

$$
\mathcal{P}:=\left(\begin{array}{cc}
0 & \mathbb{I}_{3} \\
\mathbb{I}_{3} & 0
\end{array}\right)
$$

implements the inversion symmetry defined with the help of Fig.9(a). To define the inversion symmetry, we first draw the dashed line in Fig. 9(a). A cut along this dashed line defines an arm-chair boundary. We then select the intersection of the dashed line with the mid-point of the bond emerging from the site 1 of the enlarged repeat unit cell below the dashed line in Fig. 9(a), This mid-point, represented by a filled circle in Fig. 9(a), defines the inversion center. Performing an inversion about this point maps the Kekule pattern below the dashed line into the Kekule pattern above the dashed line. The two patterns are identical, hence the inversion symmetry. On the one hand, the labels in the enlarged repeat unit cell below the dashed line becomes those above the dashed line in Fig. 9(a) under this inversion. On the other hand, if the convention for the labels of the enlarged repeat unit cell are identical below and above the dashed line, the representation $7.6 \mathrm{~b}$ ) follows.

Observe that

$$
\{\mathcal{S}, \mathcal{P}\}=0 \text {. }
$$

We will make use of this anti-commutator in Sec. (7.5.4).

\subsection{Partition}

A slab geometry is cut from Fig. 9(a) by choosing two armchair edges running parallel to the $\boldsymbol{a}_{1}$ direction of the two-dimensional embedding Euclidean space. Periodic boundary conditions are imposed along the $\boldsymbol{a}_{1}$ direction, open ones along the $\boldsymbol{a}_{2}$ direction. Hence, the momentum $k_{1}$ is a good quantum number.

This slab geometry with the choice of mixed periodic and open boundary conditions is identical to the cylindrical geometry shown in Fig. 6. The dashed line in Fig. 9(a) can be identified with the intersection of the red plane with the cylinder in Fig. 6. The dashed line in Fig. 9(a) will shortly be identified with an entangling boundary that is invariant under the inversion symmetry about the inversion center in Fig. 9(a). 


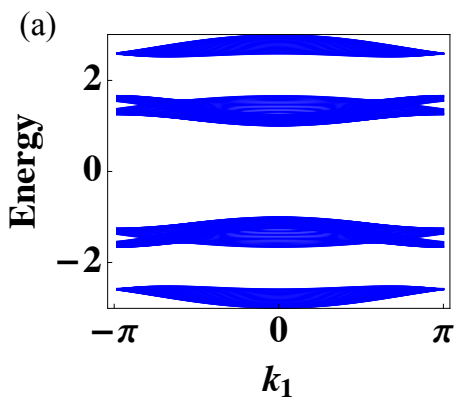

(c)

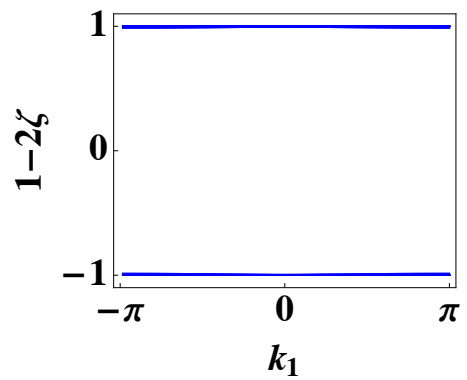

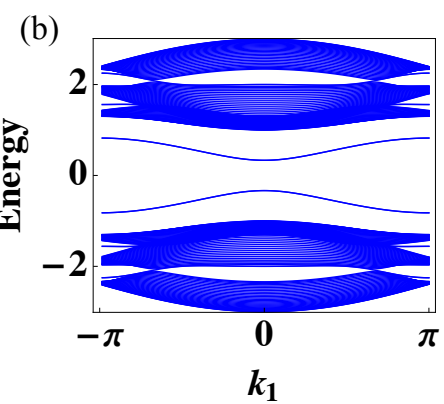

(d)

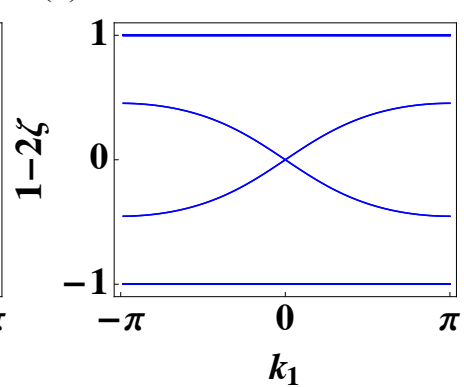

Figure 10. (Color online) Energy spectrum of Hamiltonian (7.1) with armchair edges for (a) $\left(t_{1}, t_{2}\right)=(1 / 3,4 / 3)$ and (b) $\left(t_{1}, t_{2}\right)=(5 / 3,2 / 3)$. Entanglement spectrum with armchair entangling edges for (c) $\left(t_{1}, t_{2}\right)=(1 / 3,4 / 3)$ and (d) $\left(t_{1}, t_{2}\right)=(5 / 3,2 / 3)$. The dimensions of the lattice are given by $\left(N_{1}, N_{2}\right)=(128,32)$, where $N_{i}$ is the number of the repeat unit cell from Fig. 9(a) along the direction of the spanning vector $\boldsymbol{a}_{i}(i=1,2)$ and in units for which the spanning vectors $\boldsymbol{a}_{1}$ and $\boldsymbol{a}_{2}$ are of unit length.

We use the partition introduced in Sec. 5.3 with the convention that the good quantum number is $k_{1}$, i.e., with $i=1$ in Sec.5.3. The single-particle Hilbert space is the direct sum

$$
\mathfrak{H}=\bigoplus_{k_{1}} \mathfrak{H}_{k_{1}}
$$

For any good momentum quantum number in the one-dimensional Brillouin zone

$$
k_{1}:=\frac{2 \pi}{2 M_{1}\left|\boldsymbol{a}_{1}\right|} n_{1}, \quad n_{1}=1, \cdots, 2 M_{1}
$$

where $N_{1}=2 M_{1}$ is the number of enlarged unit cells along the $\boldsymbol{a}_{1}$ direction, the subspace $\mathfrak{H}_{k_{1}}$ is spanned by the orthonormal single-particle states

$$
\left\{\left|k_{1}, n_{2}, \alpha\right\rangle \mid n_{2}=1, \cdots, 2 M_{2}, \quad \alpha=1, \cdots, 6\right\}
$$

with $n_{2}$ labeling the $N_{2}=2 M_{2}$ enlarged unit cells in the direction $\boldsymbol{a}_{2}$ and $\alpha=1, \cdots, 6$ labeling the inequivalent sites within an enlarged unit cell. The single-particle Hamiltonian $\mathcal{H}_{k_{1}}$ has matrix elements of the form given in Eq. 5.9b. The partition for any given good quantum number $k_{1}$ is then

$$
\mathfrak{H}_{k_{1}}:=\mathfrak{H}_{A_{2}} \oplus \mathfrak{H}_{B_{2}}
$$


where

$$
\mathfrak{H}_{A_{2}}:=\bigoplus_{n_{2}=1}^{M_{2}} \bigoplus_{\alpha=1}^{6}\left|k_{1}, n_{2}, \alpha\right\rangle\left\langle k_{1}, n_{2}, \alpha\right|
$$

and

$$
\mathfrak{H}_{B_{2}}:=\bigoplus_{n_{2}=M_{2}+1}^{2 M_{2}} \bigoplus_{\alpha=1}^{4}\left|k_{1}, n_{2}, \alpha\right\rangle\left\langle k_{1}, n_{2}, \alpha\right| .
$$

If we denote by $\mathscr{P}$ the inversion about the inversion center in Fig. 9(a) that reverses the sign of the good quantum number $k_{1}$, i.e.,

$$
\mathscr{P} k_{1}=-k_{1} \text {, }
$$

we then have that

$$
\mathscr{P} A_{2}=B_{2} \text {, }
$$

i.e., $\mathscr{P}$ interchanges the physical boundaries while it leaves the entangling boundary between $A_{2}$ and $B_{2}$ invariant as a set.

\subsection{Kekule with armchair edges}

Both the energy and entanglement spectra are obtained by exact diagonalization with $\left(N_{1}, N_{2}\right)=(128,32)$ and presented in Fig. 10 for different values of $t_{1}$ and $t_{2}$ in Eq. (7.1).

The energy spectrum is bulk-like when $t_{1}<t_{2}$ as is illustrated with Fig. 10(a). Edge modes are not present when $t_{1}<t_{2}$ in the energy spectrum, Fig. 10(a) being an example of this observation. For each armchair boundary, The energy spectrum supports a single pair of right- and left-moving edge states when $t_{1}>t_{2}$, as is illustrated with Fig. 10.(b). These edge states do not cross, they are gaped at the band center.

The entanglement spectrum is bulk-like when $t_{1}<t_{2}$ as is illustrated with Figs. 10.(c). Edge modes are not present when $t_{1}<t_{2}$ in the entanglement spectrum, Fig. 10(c) being an example of this observation. For each armchair boundary, the entanglement spectrum supports a single pair of right- and left-moving edge states when $t_{1}>t_{2}$, as is illustrated with Fig. 10(d). These edge states cross at the band center.

In the following, we shall choose $\left(t_{1}, t_{2}\right)=(5 / 3,2 / 3)$ and study the robustness of the crossing of the edge states in the entanglement spectrum in the presence of three symmetry-breaking perturbations. We either break time-reversal symmetry, chiral symmetry, or inversion symmetry once at a time. Spectra obtained by exact diagonalization are presented in Fig. 11, i.e., we study the spectra of

$$
\hat{H}:=\hat{H}_{\mathrm{K}}+\hat{H}^{\prime}
$$

with $\hat{H}^{\prime}$ a one-body perturbation that breaks either time-reversal symmetry, chiral symmetry, or inversion symmetry. 


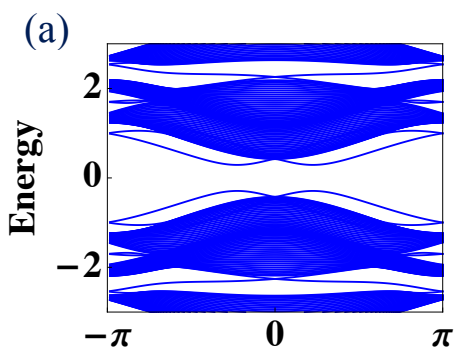

$k_{1}$

(d)

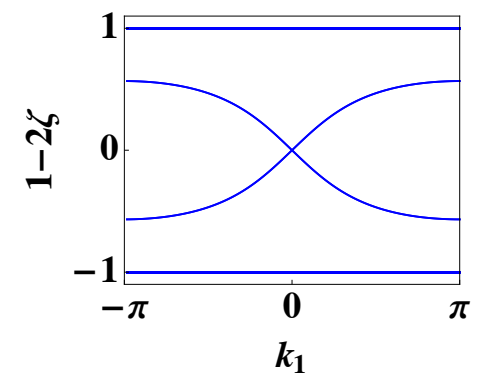

(b)

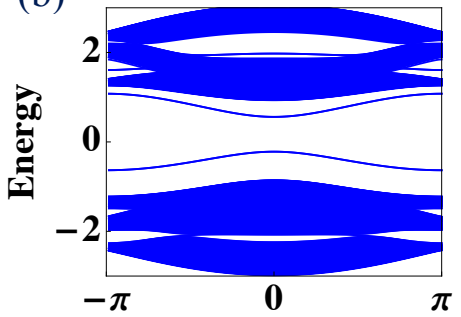

$k_{1}$

(e)

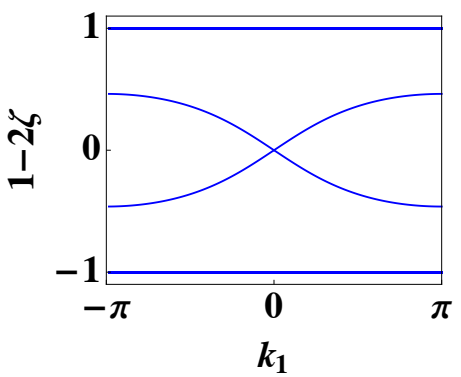

(c)

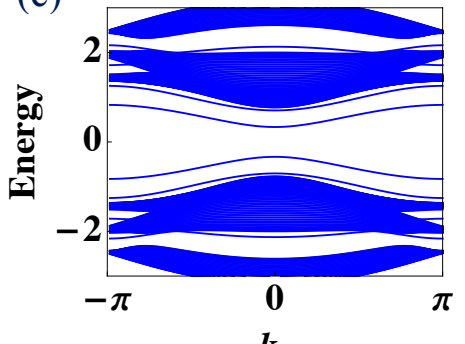

(f)

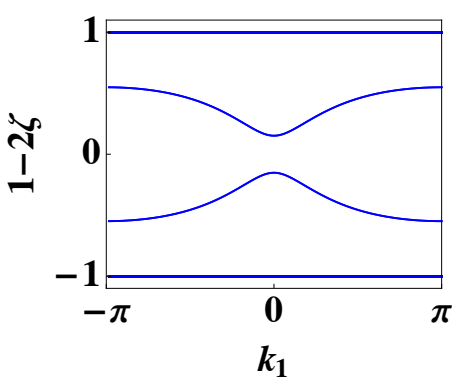

Figure 11. (Color online) Energy spectra are presented in the left column, entanglement spectra are presented in the right column. In both cases, the geometry is that of a slab with armchair edges as in Fig. 9(a) for $\left(t_{1}, t_{2}\right)=(5 / 3,2 / 3)$ and $\left(N_{1}, N_{2}\right)=(128,32)$. The spectra (a) and (d) are obtained by choosing the time-reversal-breaking perturbation 7.13 in Hamiltonian (7.12). The spectra (b) and (e) are obtained by choosing the chiralsymmetry-breaking perturbation 7.14 with 7.16 in Hamiltonian 7.12. The spectra (c) and (f) are obtained by choosing the inversion-symmetry-breaking perturbation (7.17) to Hamiltonian (7.12).

7.5.1. Time-reversal symmetry breaking We choose in Eq. (7.12) the perturbation defined by

$$
\mathcal{H}^{\prime}:=l\left(\begin{array}{cccccc}
0 & e^{\mathrm{i} \phi} & 0 & 0 & 0 & e^{-\mathrm{i} \phi} \\
e^{-\mathrm{i} \phi} & 0 & e^{\mathrm{i} \phi} & 0 & 0 & 0 \\
0 & e^{-\mathrm{i} \phi} & 0 & e^{\mathrm{i} \phi} & 0 & 0 \\
0 & 0 & e^{-\mathrm{i} \phi} & 0 & e^{\mathrm{i} \phi} & 0 \\
0 & 0 & 0 & e^{-\mathrm{i} \phi} & 0 & e^{\mathrm{i} \phi} \\
e^{\mathrm{i} \phi} & 0 & 0 & 0 & e^{-\mathrm{i} \phi} & 0
\end{array}\right),
$$

where the real number $l$ is a uniform hopping amplitude and $\phi$ is a uniform phase that breaks time-reversal symmetry if not equal to 0 or $\pi$. Figures 11(a) and 11.(b) give the physical energy and entanglement spectra, respectively, for

$$
l=0.3, \quad \phi=\pi / 4 .
$$

Both the chiral and the inversion symmetries are present for this perturbation that breaks timereversal symmetry and the spectra in Figs.11(a) and 11(d) follow. According to Fig. 11.(a) the edge states are gaped in the energy spectrum. According to 11 (d), the existence and location of the crossing of the edge states in the entanglement spectrum is seen to be robust to this perturbation. 
7.5.2. Sublattice symmetry breaking The chiral (sublattice) spectral symmetry is broken by any on-site potential. Any distribution of on-site potentials within the repeat unit cell of Fig. 9(a) that is unchanged under any linear combination of the spanning vectors $\boldsymbol{a}_{1}$ and $\boldsymbol{a}_{2}$ preserves the translation symmetry of the honeycomb lattice decorated by the colors of the Kekule strong and weak bonds. Hence, we choose in Eq. (7.12) the perturbation

$$
\mathcal{H}^{\prime}:=\operatorname{diag}\left(\mu_{1}, \mu_{2}, \mu_{3}, \mu_{4}, \mu_{5}, \mu_{6}\right),
$$

where we demand that

$$
\mu_{1}=\mu_{4}, \quad \mu_{2}=\mu_{5}, \quad \mu_{3}=\mu_{6},
$$

breaks the chiral (sublattice) spectral symmetry for any non-vanishing value of $\mu_{n}$ with $n=1, \cdots, 3$ belonging to the repeat unit cell, but preserves the reduced first Brillouin zone from Fig. 9(b) with the inversion symmetric points $\Gamma, M_{1}, M_{2}$, and $M_{3}$. Since an on-site potential is represented by a real-valued matrix, $\mathcal{H}^{\prime}$ does not break time-reversal symmetry. If we use a slab geometry with two parallel armchair edges running along the $x$ axis in the embedding two-dimensional Euclidean space, we need only demand that $k_{1}$ is a good quantum number, i.e., invariance under translation by $\boldsymbol{a}_{1}$ only. If so, we can make the choice

$$
\mu_{1}=1 / 2, \quad \mu_{2}=-1 / 4, \quad \mu_{3}=\cdots=\mu_{6}=0,
$$

for the real-valued chemical potentials within the repeat unit cell. With this choice, $k_{1}$ is a good quantum number, the time-reversal and inversion symmetries are present, but the chiral sublattice symmetry is broken, and the spectra in Figs. 11 (b) and 11(e) follow. According to Fig. 11(b) the edge states are gaped in the energy spectrum. According to 11(e), the existence and location of the crossing of the edge states in the entanglement spectrum is seen to be robust to this perturbation.

7.5.3. Inversion symmetry breaking We choose in Eq. (7.12) the perturbation

$$
\mathcal{H}^{\prime}=l\left(\begin{array}{llllll}
0 & 0 & 0 & 0 & 0 & 1 \\
0 & 0 & 0 & 0 & 0 & 0 \\
0 & 0 & 0 & 0 & 0 & 0 \\
0 & 0 & 0 & 0 & 0 & 0 \\
0 & 0 & 0 & 0 & 0 & 0 \\
1 & 0 & 0 & 0 & 0 & 0
\end{array}\right),
$$

where the real-valued

$$
l=0.5
$$

in Figs. 11(c) and 11(f). Since this perturbation is a real-valued nearest-neighbor hopping between site 1 and 6 from the repeat unit cell of Fig.9(a), time-reversal and chiral (sublattice) symmetries are present, but inversion symmetry is broken. According to Figs.11(c) and 11.f), both the energy and entanglement spectra are gaped. 
7.5.4. Stability analysis of the zero modes The two disconnected physical boundaries in the cylindrical geometry used to do the exact diagonalization presented in Figs. 10 and 11 are interchanged by the inversion symmetry. The single entangling boundary for the cylindrical geometry considered in Figs. 10 and 11 is invariant as a set under the inversion symmetry. This difference explains why there are crossings of gapless edge states in the entanglement spectra provided $\hat{H}$ defined by Eq. 7.1 with $t_{1}>t_{2}$ respects the inversion (parity) symmetry $\mathscr{P}$ (and irrespectively of the presence or absence of time reversal symmetry and spectral chiral symmetry), while there are no crossing of edge states in the energy spectrum under the same assumptions.

To explain this empirical observation deduced from exact diagonalization, we assume that the existence of a pair of left- and right-moving edge states along any one of the three armchair boundaries, namely any one of the two physical armchair boundaries and the entangling armchair boundary. We consider first the case of a single physical armchair boundary and then the case of the single entangling armchair boundary. In both cases, our effective single-particle Hamiltonian for the single pair of left and right movers on an edge is given by

$$
\mathcal{H}_{\text {edge }}=\bigoplus_{k} \mathcal{H}_{\text {edge } k}
$$

where the momentum along the edge is denoted by $k$ and

$$
\mathcal{H}_{\text {edge } k}=v_{0} \sigma_{0}+v_{1} \sigma_{1}+v_{2} \sigma_{2}+k \sigma_{3}
$$

to leading order in a gradient expansion. Here, we have introduced the usual suspects, namely the $2 \times 2$ unit matrix $\sigma_{0}$ and the three Pauli matrices $\sigma_{1}, \sigma_{2}$, and $\sigma_{3}$ to which we associate three energy scales through the real numbers $v_{0}, v_{1}, v_{2}$, and one group velocity $v_{3}$ that we have set to unity ( $\hbar=1$ as well), respectively.

Energy spectrum The effective Hamiltonian (7.18) for a single physical armchair boundary inherits two symmetries from Hamiltonian (7.1) (the case $t_{1}>t_{2}$ is assumed to have edge states). There is the symmetry $\mathscr{T}_{\text {edge }}$ under reversal of time. There is the spectral symmetry $\mathscr{S}$ edge under multiplication by a minus sign of all the single-particle states in the position basis on one and only one of the two triangular sublattices of the honeycomb lattice. There is no inversion symmetry generated by a putative operator $\mathscr{P}_{\text {edge }}$ for a single physical armchair boundary. Because we have been considering spinless fermions, a representation of $\mathscr{T}_{\text {edge }}$ is uniquely fixed by demanding that reversal of time interchanges left and right movers while squaring to unity as an insanitary operator, i.e.,

$$
\mathcal{T}_{\text {edge }}:=\sigma_{1} K
$$

where $K$ denotes complex conjugation. Symmetry under reversal of time is the condition

$$
\mathcal{H}_{\text {edge }+k}=\sigma_{1} \mathcal{H}_{\text {edge }-k}^{*} \sigma_{1}
$$


that is met for any $v_{0}, v_{1}$, and $v_{2}$ in Eq. (7.18). Hence, it is only the sublattice symmetry that restricts the allowed values of $v_{0}, v_{1}$, and $v_{2}$ in Eq. (7.18). There are two possible choices to represent $\mathscr{S}_{\text {edge }}$ by a unitary matrix $\mathcal{S}_{\text {edge }}$ such that

$$
\mathcal{H}_{\text {edge } k}=-\mathcal{S}_{\text {edge }} \mathcal{H}_{\text {edge } k} \mathcal{S}_{\text {edge }}^{-1},
$$

namely

$$
\mathcal{S}_{\text {edge }}=\sigma_{1}
$$

or

$$
\mathcal{S}_{\text {edge }}=\sigma_{2} .
$$

In both cases, the spectral symmetry (7.21) fixes the chemical potential to the value $v_{0}=0$. In the former case, the spectral symmetry with the generator (7.21b) fixes $v_{1}=0$ but leaves $v_{2}$ arbitrary so that a gap opens up as soon as $v_{2} \neq 0$ in the energy spectrum of the edge states. In the latter case, the spectral symmetry with the generator (7.21c fixes $v_{2}=0$ but leaves $v_{1}$ arbitrary so that a gap opens up as soon as $v_{1} \neq 0$ in the energy spectrum of the edge states.

Entanglement spectrum We now assume that the single pair of left and right movers propagating along the entangling boundary is governed by the effective

$$
\mathcal{Q}_{\text {edge } A k} \equiv \mathcal{H}_{\text {edge } k}
$$

with $\mathcal{H}_{\text {edge } k}$ defined in Eq. (7.18). The symmetries obeyed by $\mathcal{Q}_{\text {edge } A k}$ are

$$
\begin{aligned}
& \mathcal{Q}_{\text {edge } A+k}=\mathcal{T}_{\text {edge }} \mathcal{Q}_{\text {edge } A-k}^{*} \mathcal{T}_{\text {edge }}^{-1}, \\
& \mathcal{Q}_{\text {edge } A k}=-\mathcal{S}_{\text {edge }} \mathcal{Q}_{\text {edge } A k} \mathcal{S}_{\text {edge }}^{-1}, \\
& \mathcal{Q}_{\text {edge } A+k}=-\Gamma_{\mathcal{P}_{\text {edge }}} \mathcal{Q}_{\text {edge } A-k} \Gamma_{\mathcal{P}_{\text {edge }}}^{-1},
\end{aligned}
$$

[Recall Eq. $2.52 \mathrm{~b}]$ ]. Needed is a representation of the unitary generator $\Gamma_{\mathcal{P}_{\text {edge }}}$ for inversion on the entangling boundary. Since

$$
k \sigma_{3}=-\sigma_{\mu}\left(-k \sigma_{3}\right) \sigma_{\mu}
$$

for both $\mu=0$ or $\mu=3$, there seems to be an ambiguity when defining $\Gamma_{\mathcal{P}_{\text {edge }}}$. On the one hand, choosing

$$
\Gamma_{\mathcal{P}_{\text {edge }}}=\sigma_{0}
$$

implies that $\Gamma_{\mathcal{P}_{\text {edge }}}$ commutes with either choices $(7.21 \mathrm{~b})$ or $(7.21 \mathrm{c})$. On the other hand, choosing

$$
\Gamma_{\mathcal{P}_{\text {edge }}}=\sigma_{3}
$$

implies that $\Gamma_{\mathcal{P}_{\text {edge }}}$ anti-commutes with either choices $77.21 \mathrm{~b}$ or $77.21 \mathrm{c}$. Now, it is only when $\Gamma_{\mathcal{P}_{\text {edge }}}$ commutes with $\mathcal{S}_{\text {edge }}$ that inversion symmetry protects the crossing of the pair of edge states at the band center by fixing $v_{0}=v_{1}=v_{2}=0$ irrespectively of whether sublattice symmetry holds or not! The ambiguity in choosing between the representations 
(7.25a) and (7.25b) is spurious, however. We must choose the representation (7.25a) as we now demonstrate.

We are now going to show that irrespective of the choice made to represent $\mathscr{S}_{\text {edge }}$, we must choose to represent $\mathscr{P}_{\text {edge }}$ such that

$$
\left[\mathcal{S}_{\text {edge }}, \Gamma_{\mathcal{P}_{\text {edge }}}\right]=0 .
$$

To this end, we use the fact that the chiral transformation representing the spectral sublattice symmetry and that representing the inversion symmetry in the bulk, recall Eq. (7.5) and (7.6), anti-commute according to Eq. (7.7).

In the presence of inversion symmetry, there is a spectral symmetry $\Gamma_{\mathscr{P}}$ in the entanglement spectrum. This spectral symmetry was defined by $\Gamma_{\mathscr{P}}=C_{A B k} P$ in Eq. 2.45d (we are using the conventions of Sec. 2.1.4. for the choice of the fonts of the symmetry generators). In the presence of the spectral symmetry

$$
S Q_{A k} S^{-1}=-Q_{A k}
$$

and

$$
S C_{A B k} S^{-1}=-C_{A B k}
$$

with the anti-commutator $\{S, P\}=0$ in agreement with Eq. (7.7). We then have

$$
\begin{aligned}
{\left[S, \Gamma_{\mathscr{P}}\right] } & =\left[S, C_{A B k} P\right] \\
& =\left\{S, C_{A B k}\right\} P-C_{A B k}\{S, P\} \\
& =0 .
\end{aligned}
$$

We have constructed the explicit representations of $S$ and $\Gamma_{\mathscr{P}}$ obtained after exact diagonalization of Eq. (7.12) and verified that Eq. (7.29) holds.

\subsection{Rotated Kekule with armchair edges}

The honeycomb lattice is unchanged under rotations by $\pi / 3$ about the center of an elementary hexagon. The Kekule order breaks this point-group symmetry down to rotations by $2 \pi / 3$ about the center of an elementary hexagon. This pattern of symmetry breaking is that of $C_{6} \rightarrow C_{3}$, where $C_{n}$ is the $n$-fold rotation symmetry group. Rotations by arbitrary angles about a point in a plane form a group, the Abelian group $U(1)$.

This suggests a connection between the Kekule order parameter and the spontaneous breaking of an internal symmetry group $U(1)$. This connection becomes precise in the approximation by which the spectrum of graphene is linearized about the Dirac points. The actions of rotations about the center of an hexagon in graphene involve, in the Dirac approximation, a mixing of the components of the Dirac spinors through the action of one of the Dirac matrices denoted $\gamma_{5}$. In the terminology of high-energy physics, $\gamma_{5}$ is associated to a pseudoscalar charge called the axial charge. For graphene, this pseudoscalar charge is the difference in the local density of electrons associated to the two valleys of graphene. 


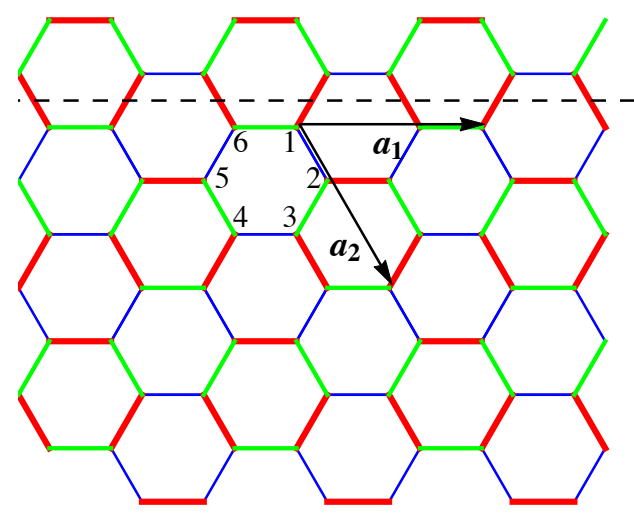

(a)

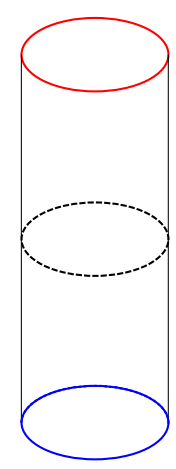

(b)

Figure 12. (Color online) (a) The nearest-neighbor bonds of the honeycomb lattice are colored in red, blue, and green as depicted. The colors red, blue, and green correspond to the values $t_{1}, t_{2}$, and $t_{3}$ taken by the nearest-neighbor hopping amplitudes for spinless fermions hopping on the honeycomb lattice with Hamiltonian (7.30), respectively. The repeat unit cell with its spanning vectors $\boldsymbol{a}_{1}$ and $\boldsymbol{a}_{2}$ was defined in Fig.9(a) An armchair entangling edge is obtained by opening the honeycomb lattice through the dashed line. (b) The cylindrical geometry with the two armchair edges differing by their colors is selected by imposing periodic boundary conditions along the $a_{1}$ direction and open ones along the $a_{2}$ direction. The top armchair edge denoted by a red ellipse has fermions hopping along it with the consecutive hopping amplitudes $t_{1}, t_{2}, t_{3}$, and $t_{2}$. The bottom armchair edge denoted by the blue ellipse has fermions hopping along it with the consecutive hopping amplitudes $t_{1}, t_{3}, t_{2}$, and $t_{3}$. [Note that these are not the armchair boundaries shown in panel (a).]

In the Dirac limit, the pattern of symmetry breaking induced by a Kekule distortion becomes the spontaneous breaking of a continuous $U(1)$ symmetry generated by the axial gauge charge.

The Kekule distortion can support a point defect at which three Kekule distortions differing pairwise by a global axial phase of either $2 \pi / 3$ or $4 \pi / 3(\bmod 2 \pi)$ meet. With open boundary conditions, such a point defect was shown two support two localized zero modes. There is one zero mode localized around the point defect. There is one zero mode localized somewhere on the boundary. The location on the boundary of the latter zero mode depends on the value taken by the global axial phase of the defective Kekule distortion.

Our purpose is to study the influence of the choice made for the global axial phase of a uniform Kekule distortion on the spectrum of the Kekule Hamiltonian with armchair open boundary conditions.

7.6.1. Rotated Kekule distortion To parametrize a global rotation of a Kekule distortion, we define

$$
\hat{H}_{\mathrm{K}}(\alpha):=\sum_{n=1,2,3} t_{n}(\alpha) \sum_{\left\langle\boldsymbol{i}_{n} \boldsymbol{j}_{n}\right\rangle}\left(\hat{c}_{\boldsymbol{i}_{n}}^{\dagger} \hat{c}_{\boldsymbol{j}_{n}}+\text { H.c. }\right) \text {, }
$$



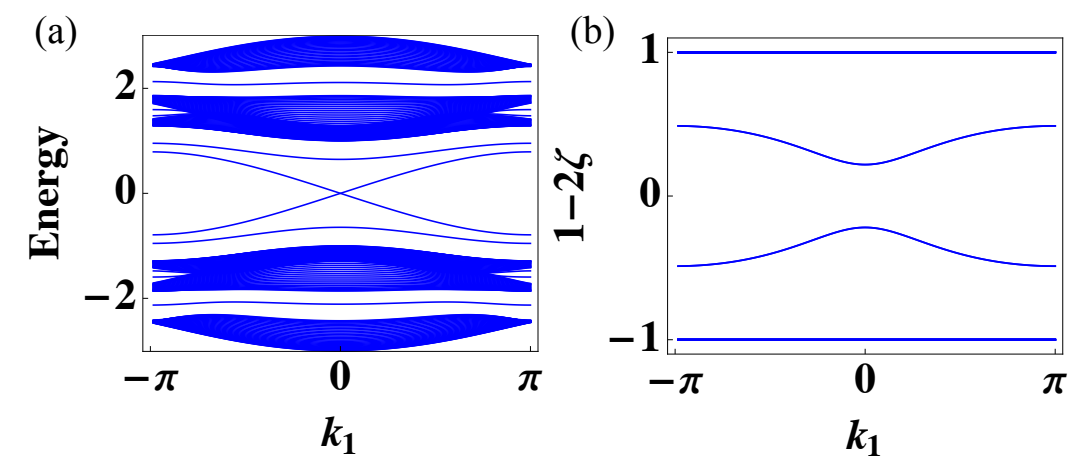

Figure 13. (Color online) (a) Energy spectrum with two armchair edges in the cylindrical geometry of Fig. 12(b) (b) Entanglement spectrum with two entangling armchair boundaries in a toroidal geometry. The energy scales are $\Delta_{0}=1$ and $t=-1$. The axial phase is $\alpha \cong 5.927$. The lattice size is $\left(N_{1}, N_{2}\right)=(128,32)$. The number of unit cells along $\boldsymbol{a}_{i}$ is $N_{i}$ for $i=1,2$.

where

$$
t_{n}(\alpha):=-t+\frac{1}{3}\left(\Delta_{\mathrm{K}}(\alpha) e^{i(n-1) 2 \pi / 3}+\Delta_{\mathrm{K}}^{*}(\alpha) e^{-i(n-1) 2 \pi / 3}\right)
$$

and

$$
\Delta_{\mathrm{K}}(\alpha):=\Delta_{\mathrm{K} 0} e^{+\mathrm{i} \alpha} .
$$

Here, $t$ is real-valued and the Kekule distortion has the amplitude $\Delta_{\mathrm{K} 0} \geq 0$ and global axial phase $0 \leq \alpha<2 \pi$. Moreover, a pair of nearest-neighbor sites of the honeycomb lattice is denoted by $\left\langle\boldsymbol{i}_{i} \boldsymbol{j}_{i}\right\rangle$ if they are connected by a bond colored in red $(i=1)$, blue $(i=2)$, and green $(i=3)$, respectively as is depicted in Fig. 12(a),

On a torus, two-dimensional momentum $\boldsymbol{k} \equiv\left(k_{1}, k_{2}\right)$ is a good quantum number and the single-particle Hamiltonian with a rotated Kekule distortion becomes (the $\alpha$ dependence is implicit)

$$
\mathcal{H}_{\boldsymbol{k}}=\left(\begin{array}{cccccc}
0 & t_{2} & 0 & t_{1} e^{-\mathrm{i}\left(k_{2}-k_{1}\right)} & 0 & t_{3} \\
t_{2} & 0 & t_{3} & 0 & t_{1} e^{+\mathrm{i} k_{1}} & 0 \\
0 & t_{3} & 0 & t_{2} & 0 & t_{1} e^{+\mathrm{i} k_{2}} \\
t_{1} e^{+\mathrm{i}\left(k_{2}-k_{1}\right)} & 0 & t_{2} & 0 & t_{3} & 0 \\
0 & t_{1} e^{-\mathrm{i} k_{1}} & 0 & t_{3} & 0 & t_{2} \\
t_{3} & 0 & t_{1} e^{-\mathrm{i} k_{2}} & 0 & t_{2} & 0
\end{array}\right) .
$$

For vanishing global axial phase, Hamiltonian (7.31) reduces to Hamiltonian (7.3b). Hamiltonian (7.31) is invariant under the same operation for time-reversal as was the case for Hamiltonian (7.3b). The spectrum of Hamiltonian (7.31) is invariant under the same chiral (sublattice) operation as was the case for Hamiltonian (7.3b). Hamiltonian (7.31) breaks the inversion symmetry enjoyed by Hamiltonian (7.3b) for any $\alpha \neq 0 \bmod 2 \pi$. This can be seen by inspection of the armchair boundaries in Fig. 12(a) and is indicated in Fig. 12(b) by the distinct colors used to denote the two edges if a cylindrical geometry is selected by the choice of boundary conditions with the direction $\boldsymbol{a}_{2}$ the open direction. 
7.6.2. Spectra for rotated Kekule The energy spectrum as a function of the good momentum quantum number $k_{1}$ is computed by diagonalizing the Hamiltonian (7.30) in the cylindrical geometry of Fig. 12(b) For any $\alpha \neq 0$ modulo $2 \pi$, inversion symmetry is broken so that the quantum dynamics on the opposite edges of the cylinder in Fig. 12(b) differ. Remarkably, at a critical value of the axial angle $\alpha_{\mathrm{c}}$, the gap for the single pair of left- and right-movers on one of the edges closes, while it does not for the single pair of left- and right-movers from the other edge. This property of the energy spectrum of the rotated Kekule distortion is illustrated in Fig. 13 (a). The critical value of the axial angle $\alpha_{\mathrm{c}}$ is $\alpha_{\mathrm{c}} \approx 5.927$ when $\left(\Delta_{\mathrm{K} 0}, t\right)=(1,-1)$, as we now show. To this end, we use the $4 \times 4$ Hamiltonians

$$
\mathcal{H}_{\mathrm{top} k_{1}}^{\text {edge }}(\alpha):=\left(\begin{array}{cccc}
0 & t_{1}(\alpha) & 0 & t_{2}(\alpha) e^{+\mathrm{i} k_{1}} \\
t_{1}(\alpha) & 0 & t_{2}(\alpha) & 0 \\
0 & t_{2}(\alpha) & 0 & t_{3}(\alpha) \\
t_{2}(\alpha) e^{-\mathrm{i} k_{1}} & 0 & t_{3}(\alpha) & 0
\end{array}\right)
$$

and

$$
\mathcal{H}_{\text {bot } k_{1}}^{\text {edge }}(\alpha):=\left(\begin{array}{cccc}
0 & t_{1}(\alpha) & 0 & t_{3}(\alpha) e^{+\mathrm{i} k_{1}} \\
t_{1}(\alpha) & 0 & t_{3}(\alpha) & 0 \\
0 & t_{3}(\alpha) & 0 & t_{2}(\alpha) \\
t_{3}(\alpha) e^{-\mathrm{i} k_{1}} & 0 & t_{2}(\alpha) & 0
\end{array}\right)
$$

to model hopping restricted to the top and bottom armchair boundaries defined in Fig. 14 , respectively. For the top armchair edge, we are using four orbitals per repeat unit cell with the conventions that orbital 1 hops to orbital 2 with the amplitude $t_{1}(\alpha)$, orbital 2 hops to orbital 3 with the amplitude $t_{2}(\alpha)$, orbital 3 hops to orbital 4 with the amplitude $t_{3}(\alpha)$, and orbital 4 hops to orbital 1 in the neighboring repeat unit cell with the amplitude $t_{2}(\alpha)$. For the bottom armchair edge, we are using the conventions that follow from those for the top armchair edge obtained by exchanging $t_{2}(\alpha)$ and $t_{3}(\alpha)$. Eigenstates of Hamiltonian $\mathcal{H}_{\text {top } k_{1}}^{\text {edge }}(\alpha)$ at $k_{1}=0$ with zero energy eigenvalue satisfy $\operatorname{det}\left[\mathcal{H}_{\text {top } k_{1}=0}^{\text {edge }}(\alpha)\right]=0$. This condition gives $\alpha_{\mathrm{c}} \approx 5.927$ when $\left(\Delta_{\mathrm{K} 0}, t\right)=(1,-1)$ for the critical axial angle.

We have also calculated the entanglement spectrum using the same partition as the one used in Sec. 7.4, with the proviso that we are now using the dashed line shown in Fig. 12(a), To avoid contamination in the entanglement spectrum arising from the gap closing along one of the physical edges when $\alpha=\alpha_{\mathrm{c}}$, we choose to impose full periodic boundary conditions, i.e., the geometry of a torus. We expect no closing of the entanglement spectrum because of the breaking of inversion symmetry by any $\alpha \neq 0$ modulo $2 \pi$ and indeed, this is what is observed from exact diagonalization and illustrated with the help of Fig. 13 (b).

\subsection{Kekule with zigzag edges}

7.7.1. Hamiltonian The energy spectrum of semi-infinite graphene modeled by a single nearest-neighbor hopping amplitude on the honeycomb lattice with a zigzag edge shows flat (dispersionless) bands connecting the two Dirac points. [51] These zero-energy flat bands 


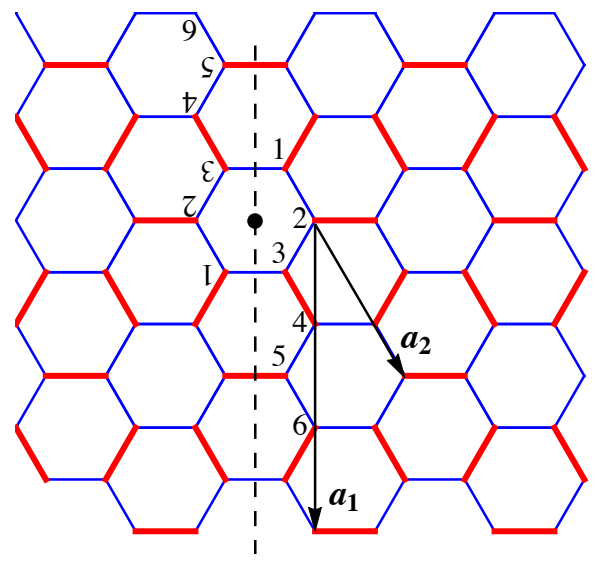

Figure 14. (Color online) The nearest-neighbor bonds of the honeycomb lattice are colored in red and blue as depicted. The colors red and blue correspond to the values $t_{1}$ and $t_{2}$ taken by the nearest-neighbor hopping amplitudes for spinless fermions hopping on the honeycomb lattice with Hamiltonian 7.34, respectively. A Kekule distortion follows from choosing $t_{1} \neq t_{2}$. A physical zigzag edge is constructed from cutting through the dashed line. The dashed line also defines a zigzag entangling edge. The symbol $\bullet$ denotes an inversion center. One repeat unit cell contains three hexagons defined as follows. The first hexagon from the repeat unit cell has two sites numbered 1 and 2, whereby site 1 is connected by a blue bond to site 2 . The second hexagon from the repeat unit cell has three sites numbered 2, 3, and 4, whereby site 2 is connected by a blue bond to site 3 , while site 3 is connected by a red bond to site 4 . The third hexagon from the repeat unit cell has all six edges colored in blue with the vertices numbered 4,5 , and 6 . The spanning vectors corresponding to this unit cell are $\boldsymbol{a}_{1}$ and $\boldsymbol{a}_{2}$. The image of the repeat unit cell under inversion about the point $\bullet$ is has its three hexagons labeled with the numbers 1 to 6 written upside down.

are protected by chiral symmetry, see Ref. [52]. These flat bands can become dispersive by tuning on when perturbed by a one-body potential that breaks the inversion symmetry, as demonstrated in Ref. [53]. We consider the energy and entanglement spectra of graphene with a Kekule distortion in the presence of physical and entangling zigzag edges. The repeat unit cell and the spanning vectors are defined in Fig 14. With the conventions of Fig 14, the BZ and four inversion symmetric momenta are identical to the BZ and four inversion symmetric momenta from Fig. 9(b) if we impose periodic boundary conditions.

The single particle Hamiltonian in momentum space is

$$
\mathcal{H}_{\text {zig } \boldsymbol{k}}=\left(\begin{array}{cccccc}
0 & t_{2} & 0 & t_{2} e^{-\mathrm{i} k_{2}} & 0 & t_{1} e^{-\mathrm{i} k_{1}} \\
t_{2} & 0 & t_{2} & 0 & t_{1} e^{+\mathrm{i}\left(k_{2}-k_{1}\right)} & 0 \\
0 & t_{2} & 0 & t_{1} & 0 & t_{2} e^{-\mathrm{i} k_{2}} \\
t_{2} e^{+\mathrm{i} k_{2}} & 0 & t_{1} & 0 & t_{2} & 0 \\
0 & t_{1} e^{-\mathrm{i}\left(k_{2}-k_{1}\right)} & 0 & t_{2} & 0 & t_{2} \\
t_{1} e^{+\mathrm{i} k_{1}} & 0 & t_{2} e^{+\mathrm{i} k_{2}} & 0 & t_{2} & 0
\end{array}\right) .
$$

\subsubsection{Symmetries The symmetry}

$$
\mathcal{H}_{\mathrm{zig}-\boldsymbol{k}}^{*}=\mathcal{H}_{\mathrm{zig}+\boldsymbol{k}}
$$




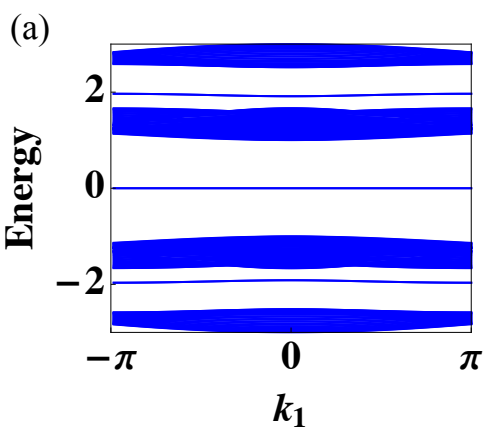

(c)

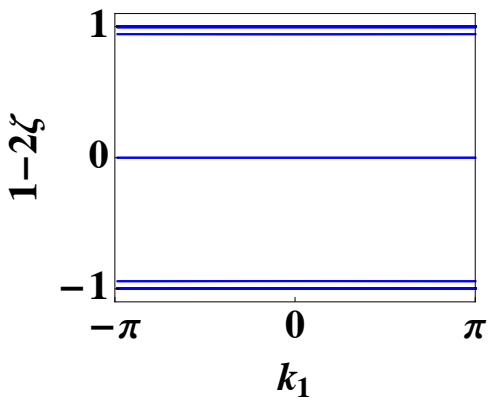

(b)

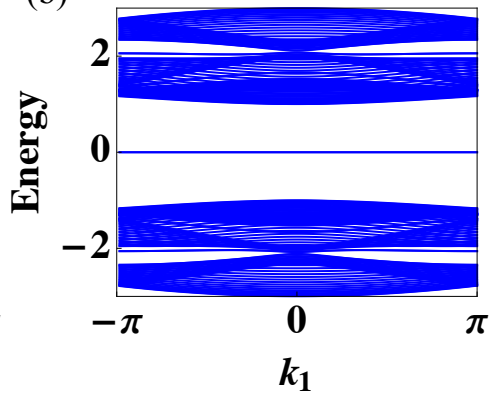

(d)

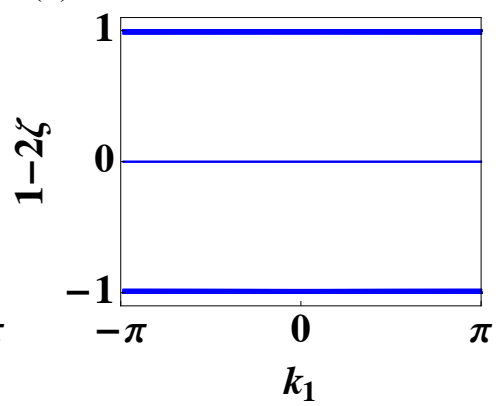

Figure 15. (Color online) Energy spectra of $\mathcal{H}_{\mathrm{zig} k_{1}}$ in Eq. 7.39 with zigzag edges in a cylinder geometry for (a) $\left(t_{1}, t_{2}\right)=(1 / 3,4 / 3)$ and (b) $\left(t_{1}, t_{2}\right)=(5 / 3,2 / 3)$. Entanglement spectra of $\mathcal{H}_{\text {zig } k_{1}}$ in Eq. 7.39 with zigzag entangling edges in a torus geometry for (c) $\left(t_{1}, t_{2}\right)=(1 / 3,4 / 3)$ and $(\mathrm{d})\left(t_{1}, t_{2}\right)=(5 / 3,2 / 3)$. The dimensions of the lattice are given by $\left(N_{1}, N_{2}\right)=(128,32)$, where $N_{i}$ is the number of the repeat unit cell from Fig. 14 along the direction of the spanning vector $\boldsymbol{a}_{i}(i=1,2)$ and in units for which the spanning vectors $\boldsymbol{a}_{1}$ and $\boldsymbol{a}_{2}$ are of unit length.

implements time-reversal symmetry for spinless fermions.

The spectral symmetry

$$
\mathcal{S}^{-1} \mathcal{H}_{\text {zig } \boldsymbol{k}} \mathcal{S}=-\mathcal{H}_{\text {zig } \boldsymbol{k}}
$$

where

$$
\mathcal{S}:=\operatorname{diag}(1,-1,1,-1,1,-1)
$$

implements the chiral (sublattice) spectral symmetry. The symmetry

$$
\mathcal{P}_{\text {zig }}^{-1} \mathcal{H}_{\text {zig }-k} \mathcal{P}_{\text {zig }}=\mathcal{H}_{\text {zig }+k},
$$

where

$$
\mathcal{P}_{\text {zig }}:=\left(\begin{array}{cccccc}
0 & 0 & 0 & 0 & 0 & 1 \\
0 & 0 & 0 & 0 & 1 & 0 \\
0 & 0 & 0 & 1 & 0 & 0 \\
0 & 0 & 1 & 0 & 0 & 0 \\
0 & 1 & 0 & 0 & 0 & 0 \\
1 & 0 & 0 & 0 & 0 & 0
\end{array}\right),
$$

implements the inversion symmetry defined with the help of Fig. 14. To define the inversion symmetry, we first draw the dashed line in Fig. 14. A cut along this dashed line defines a 
zigzag boundary. We then select the intersection of the dashed line with the mid-point of a hexagon in Fig. 14, This mid-point, represented by a filled circle in Fig. 14, defines the inversion center. Performing an inversion about this point maps the Kekule pattern on the right of the dashed line into the Kekule pattern on the left of the dashed line. The two patterns are identical, hence the inversion symmetry. On the one hand, the labels in the enlarged repeat unit cell on the right of the dashed line becomes those on the left the dashed line in Fig. 14 under this inversion. On the other hand, if the convention for the labels of the enlarged repeat unit cell are identical on the right and left of the dashed line, the representation (7.37) follows.

Observe that

$$
\left\{\mathcal{S}, \mathcal{P}_{\text {zig }}\right\}=0
$$

7.7.3. Partition The partition is defined with respect to the dashed line in Fig. 15 as was done in Sec. 7.4

\subsubsection{Hamiltonian and entanglement spectra Let}

$$
\mathcal{H}_{k_{1}}:=\mathcal{H}_{\mathrm{zig} k_{1}}+\mathcal{H}^{\prime}
$$

where $\mathcal{H}^{\prime}$ is a one-body perturbation that breaks either the chiral symmetry, the inversion symmetry, or both, and we have imposed periodic boundary conditions along the $\boldsymbol{a}_{1}$ direction from Fig. 15 so that $k_{1}$ is a good quantum number. Open boundary conditions are imposed along the $\boldsymbol{a}_{2}$ direction from Fig. 15 when computing energy spectra. Periodic boundary conditions are imposed along the $\boldsymbol{a}_{2}$ direction from Fig. 15 when computing entanglement spectra in order to avoid a spectral contamination of the entanglement boundary states arising from the zero modes from the physical boundaries.

We have studied by exact diagonalization both the energy and entanglement spectra of Hamiltonian (7.39) in a cylinder and torus geometry, respectively,

In the absence of the perturbation $\mathcal{H}^{\prime}$, non-dispersing edge states at zero energy are present for any Kekule distortion, i.e., as soon as $t_{1} \neq t_{2}$, as is illustrated in Figs. 15.(a) and 15 (b). Non-dispersing zero modes localized on the entangling boundary are also found in the entanglement spectrum for any Kekule distortion, i.e., as soon as $t_{1} \neq t_{2}$, as is illustrated in in Figs. 15(c) and 15(d).

In the following, the number of repeat unit cells are $\left(N_{1}, N_{2}\right)=(128,32)$ and we set $\left(t_{1}, t_{2}\right)=(5 / 3,2 / 3)$ when studying the robustness of the flat (entangling) edge states in the presence of three distinct $\mathcal{H}^{\prime}$. 
Inversion symmetry breaking The inversion-symmetry-breaking perturbation is chosen in Eq. 7.39 to be

$$
\mathcal{H}^{\prime}:=\left(\begin{array}{cccccc}
0 & v_{1}^{\prime} e^{+\mathrm{i} k_{1}} & 0 & 0 & 0 & 0 \\
v_{1}^{\prime} e^{-\mathrm{i} k_{1}} & 0 & 0 & 0 & 0 & 0 \\
0 & 0 & 0 & 0 & 0 & 0 \\
0 & 0 & 0 & 0 & 0 & 0 \\
0 & 0 & 0 & 0 & 0 & 0 \\
0 & 0 & 0 & 0 & 0 & 0
\end{array}\right),
$$

where $v_{1}^{\prime}=0.5$. The flat bands when $v_{1}^{\prime}=0.5$ are robust in both the energy and the entanglement spectra under this inversion-symmetry-breaking but chiral-symmetrypreserving perturbation as is shown in Fig. [16(a) and 16(d), respectively.

Chiral symmetry breaking The chiral-symmetry-breaking perturbation is chosen in Eq. (7.39) to be

$$
\mathcal{H}^{\prime}:=\operatorname{diag}\left(o_{1}, o_{2}, o_{3}, o_{3}, o_{2}, o_{1}\right),
$$

where $o_{1}=-0.13, o_{2}=0.2$, and $o_{3}=0.3$. The flat bands when $o_{1}=o_{2}=o_{3}=0$ are shifted away from zero energy in the energy spectrum shown in Fig. 16(b). However, the flat bands when $o_{1}=o_{2}=o_{3}=0$ are unchanged by $\mathcal{H}^{\prime}$ in the entanglement spectrum shown in Fig. $16(\mathrm{e})$.

Chiral symmetry and inversion symmetry breaking The inversion-symmetry-breaking and chiral-symmetry-breaking perturbation is chosen in Eq. (7.39) to be

$$
\mathcal{H}^{\prime}:=\operatorname{diag}\left(o_{1}, o_{2}, o_{3}, o_{4}, o_{5}, o_{6}\right),
$$

where $\left(o_{1}, o_{2}, o_{3}, o_{4}, o_{5}, o_{6}\right)=(0,0.1,0.2,0.3,0.2,0.1)$ in Figs. 16(c) and 16(f). This chiralsymmetry-breaking and inversion-symmetry-breaking perturbation gaps out the flat bands when $\left(o_{1}, o_{2}, o_{3}, o_{4}, o_{5}, o_{6}\right)=(0,0,0,0,0,0)$ both in the energy and entanglement spectra shown in Fig.16(c) and 16(f), respectively.

7.7.5. Stability analysis of the zero modes We have observed numerically that flat bands in $\sigma\left(\mathcal{H}_{\mathrm{zig} k_{1}}\right)$ and $\sigma\left(Q_{A k_{1}}\right)$ can only be gaped when both the chiral and inversion symmetries are broken. To understand this result, we proceed in two steps.

First, we observe numerically that (i) there is one edge state per momentum and per edge (with a wavefunction that decays exponentially fast as a function of the distance away from the edge) in both the energy and entanglement spectra in all panels from Fig. 16, (ii) this edge state is non-dispersive (independent of the good momentum quantum number $k_{1}$ ) in all panels from Fig. 16, and (iii) with vanishing eigenvalue in panels (a), (d), and (e) from Fig. 16. Properties (i) and (ii) imply that the effective edge theory on an isolated single zigzag edge of the Hamiltonian $\mathcal{H}_{\text {zig } k_{1}}$ is the momentum-resolved $1 \times 1$ matrix

$$
\mathcal{H}_{\text {edge } k_{1}}=m_{\text {energy }} \in \mathbb{R},
$$




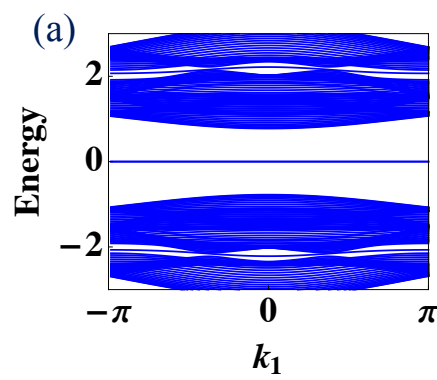

(d)

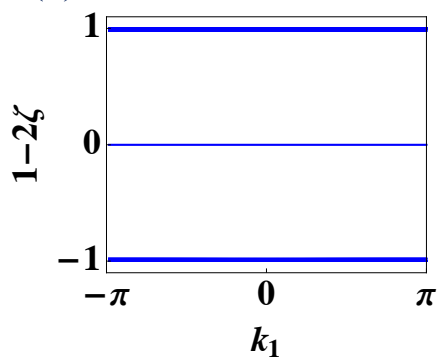

(b)

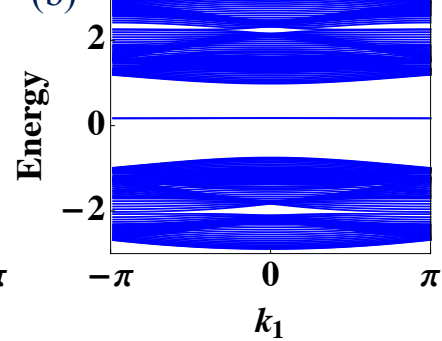

(e)

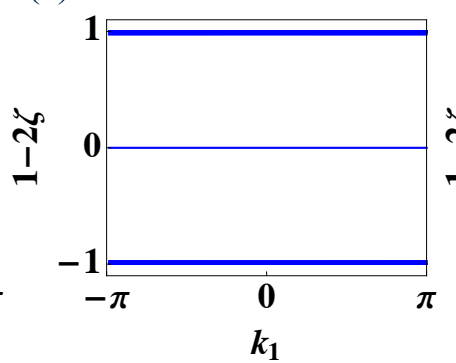

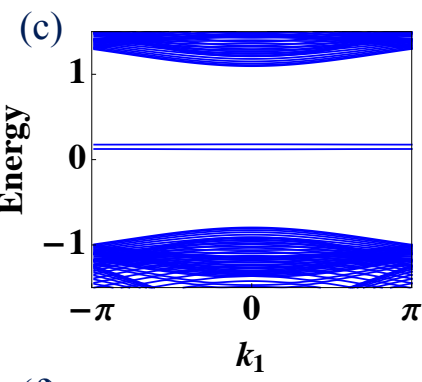

(f)

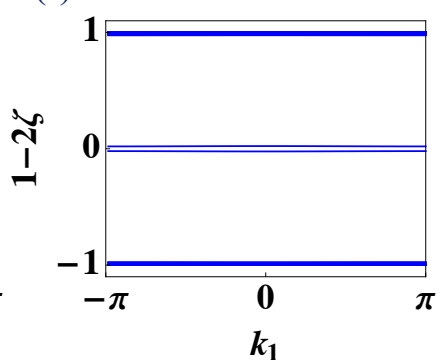

Figure 16. (Color online) Energy spectra of $\mathcal{H}_{k_{1}}$ in Eq. 7.39) with zigzag edges in a cylindrical geometry are presented in the left column. Entanglement spectra of $\mathcal{H}_{k_{1}}$ in Eq. 7.39) with zigzag entangling edges in a torus geometry are presented in the right column. In both cases, we set $\left(t_{1}, t_{2}\right)=(5 / 3,2 / 3)$ and $\left(N_{1}, N_{2}\right)=(128,32)$. The spectra (a) and (d) are obtained by choosing the inversion-breaking perturbation (7.40) in Hamiltonian 7.39). There are mid-gap flat bands that are two-fold degenerate. The spectra (b) and (e) are obtained by choosing the chiral-symmetry-breaking perturbation (7.41) in Hamiltonian 7.39). The flat bands remain two-fold degenerate but are shifted away from zero energy in panel (b). The spectra (c) and (f) are obtained by choosing both the inversion-symmetry-breaking and chiralsymmetry-breaking perturbation 7.42 to Hamiltonian 7.39 . The two-fold degeneracy of the flat bands is lifted and all flat bands are shifted away from the eigenvalue zero in panels (c) and (f).

whereas that on an isolated single zigzag edge of the entangling operator $Q_{A k_{1}}$ is the momentum-resolved $1 \times 1$ matrix

$$
Q_{\text {edge } A k_{1}}=m_{\text {entanglement }} \in \mathbb{R} \text {. }
$$

Chiral symmetry imposes the constraints

$$
\mathcal{S}_{\text {edge }}^{-1} \mathcal{H}_{\text {edge } k_{1}} \mathcal{S}_{\text {edge }}=-\mathcal{H}_{\text {edge } k_{1}}
$$

and

$$
\mathcal{S}_{\text {edge }}^{-1} Q_{\text {edge } A k_{1}} \mathcal{S}_{\text {edge }}=-Q_{\text {edge } A k_{1}} .
$$

These constraints can only be met if

$$
\mathcal{H}_{\text {edge } k_{1}}=0
$$

and

$$
Q_{\text {edge } A k_{1}}=0 .
$$


Thus, it is the spectral chiral symmetry $(7.36)$ that explains the presence of the mid-gap flat bands in panels (a) and (d) from Fig. 16. The breaking of the spectral chiral symmetry (7.36) by the perturbation (7.41) in panels (b) and (e) is manifest in the fact that the flat bands in panel (b) are not to be found anymore at the energy eigenvalue zero. The fact that the flat bands in panel (e) remains at the eigenvalue zero must be attributed to another protecting symmetry.

Second, in the presence of the inversion symmetry $(7.37)$, there exists the operator $\Gamma_{\mathscr{P}_{\text {zig }}}$ such that [recall Eq. (2.52)]

$$
\Gamma_{\mathscr{P}_{\text {zig }}} Q_{\text {edge } A+k_{1}}=-Q_{\text {edge } A-k_{1}} \Gamma_{\mathscr{P}_{\text {zig }}} .
$$

This effective chiral symmetry of the entanglement spectrum is the reason why panel (e) from Fig. 16 displays a two-fold degenerate flat band at the eigenvalue zero, whereas the two-fold degenerate flat band in panel (b) from Fig. 16 is at a non-vanishing energy eigenvalue that is determined by the amount of breaking of the spectral chiral symmetry by the perturbation (7.41). As soon as the inversion symmetry and the spectral chiral symmetry are simultaneously broken, as it is in panels (c) and (f) from Fig. 16 by the perturbation (7.42), the flat bands are to be found at non-vanishing distances from the eigenvalue zero in both the energy and the entanglement spectra, while their degeneracy has been lifted.

\subsection{Counting the mid-gap states protected by inversion symmetry}

The stability analysis of the zero modes that we have conducted so far relied on the number of edges states determined numerically before the introduction of perturbations. However, this number of zero modes can be determined analytically as follows.

It was shown in Ref. [13] that a two-dimensional topological band insulator protected by inversion symmetry is characterized by the number of zero modes $n_{A, k_{i}, k_{i}^{\star}}^{\text {zero }}$ in the entanglement spectrum $\sigma\left(Q_{A k_{i}}\right)$ defined with periodic boundary conditions (torus geometry). Here, we recall that the entangling boundary defined by the partition $A$ is characterized by the good momentum quantum number $k_{i}$ where $i=1,2$ while $i+1$ is defined modulo 2 and that $\boldsymbol{k}^{\star}=\left(k_{1}^{\star}, k_{2}^{\star}\right)$ is any momentum from the Brillouin zone that is unchanged modulo the addition of a momentum from the reciprocal lattice with the two spanning vectors $Q_{1}$ and $\boldsymbol{Q}_{2}$ under the operation of inversion. In turn, it was shown in Refs. [13] and [12] that

$$
n_{A, k_{i}, k_{i}^{\star}}^{\text {zero }}=2\left|n_{\boldsymbol{k}^{\star}}-n_{\boldsymbol{k}^{\star}+\left(\boldsymbol{Q}_{i+1} / 2\right)}\right|,
$$

where $n_{\boldsymbol{k}^{\star}}$ is the number of occupied Bloch eigenstates at the inversion symmetric momentum $\boldsymbol{k}^{\star}$ of the single-particle Hamiltonian (defined with periodic boundary conditions) that are simultaneous eigenstates of the inversion operator. The counting formula (7.47) is here meaningful because (i) $\boldsymbol{k}^{\star}$ and $\boldsymbol{k}^{\star}+\left(\boldsymbol{Q}_{i+1} / 2\right)$ are both invariant under the operation of inversion modulo the addition of a reciprocal momentum and (ii) it is possible to simultaneously diagonalize the Bloch Hamiltonian at any inversion symmetric momentum point and the operator that represents the operation of inversion. Equation (7.47) is remarkable in that it relates a property from the entangling boundary, the integer $n_{A, k_{i}, k_{i}^{\star}}^{\text {zero }}$, to a property 
of the bulk, the integer $n_{\boldsymbol{k}^{\star}}-n_{\boldsymbol{k}^{\star}+\left(\boldsymbol{Q}_{i+1} / 2\right)}$. Equation $(7.47)$ is thus an example of a bulkboundary correspondence. We choose the Fermi energy to be zero and apply Eq. (7.47) to graphene with a Kekule distortion.

Graphene with a Kekule distortion, in its simplest incarnation, has six bands that are related by the chiral operation consisting in changing the sign of the wavefunction on all the sites of one of the two triangular sublattices of the honeycomb lattice. This spectrum of graphene with a Kekule distortion is thus chiral symmetric. Hence, there are three occupied bands with strictly negative energy eigenvalues when the Fermi energy is vanishing, i.e., coincides with the mid-gap single-particle energy.

We are going to apply the counting formula (7.47) to graphene with the Kekule distortion and at a vanishing Fermi energy by choosing the inversion point to be either along an armchair cut as in Fig. 9 or along a zigzag cut as in Fig. 12. The inversion symmetric momenta from the Brillouin zone are then

$$
\boldsymbol{k}^{\star} \in\left\{(0,0)^{\top},(\pi, 0)^{\top},(0, \pi)^{\top},(\pi, \pi)^{\top}\right\}
$$

where we have chosen units such that the spanning vectors of the reciprocal lattice are $\boldsymbol{Q}_{1}=(2 \pi, 0)^{\top}$ and $\boldsymbol{Q}_{2}=(0,2 \pi)^{\top}$.

Armchair cut For the armchair case with Hamiltonian (7.3b), we assign to each of the four inversion-symmetric momenta (7.48) the row vector consisting of the three parities under the operation of inversion of the three occupied Bloch states according to

$$
\begin{array}{ll}
\Gamma\left(k_{1}=0, k_{2}=0\right): & (-,-,-), \\
M_{1}\left(k_{1}=\pi, k_{2}=0\right): & (+,-,+), \\
M_{2}\left(k_{1}=0, k_{2}=\pi\right): & (+,-,+), \\
M_{3}\left(k_{1}=\pi, k_{2}=\pi\right): & (+,-,+) .
\end{array}
$$

Hence, the number (7.47) of zero modes is four (two per entangling edge in a torus geometry) at $k_{1}=0$ and is zero at $k_{1}=\pi$ in agreement with our numerics.

Zigzag cut For the zigzag case with Hamiltonian (7.34), we assign to each of the four inversion-symmetric momenta (7.48) the row vector consisting of the three parities under the operation of inversion of the three occupied Bloch states according to

$$
\begin{array}{ll}
\Gamma\left(k_{1}=0, k_{2}=0\right): & (-,+,+), \\
M_{1}\left(k_{1}=\pi, k_{2}=0\right): & (-,-,+), \\
M_{2}\left(k_{1}=0, k_{2}=\pi\right): & (+,-,-), \\
M_{3}\left(k_{1}=\pi, k_{2}=\pi\right): & (+,-,+) .
\end{array}
$$

Hence, the number (7.47) of zero modes is two (one per entangling edge in a torus geometry) at both $k_{1}=0$ and $k_{1}=\pi$ in agreement with our numerics. 


\section{Conclusion}

The main focus of this paper has been on fermionic single-particle local Hamiltonians obeying three conditions. First, the many-body ground state is non-degenerate and incompressible if periodic boundary conditions are chosen. In short, the ground state is that of a band insulator. Second, point-group symmetries generated by non-local transformations such as a reflection or inversion must hold. Third, certain boundary conditions that are compatible with the point-group symmetries must be imposed on the entanglement spectrum through the choice of entangling boundaries. We have then constructed several examples of model Hamiltonians obeying all three conditions in one- and two-dimensional space with the following two properties. First, each model supports gapless boundary states in the entanglement spectrum that are localized on an isolated entangling boundary, even though no gapless boundary states can be found in the energy spectrum on an isolated physical boundary. Second, the stability under (one-body) perturbations of the gapless boundary states in the entanglement spectrum is guaranteed by the point-group symmetries. Common to all these examples is the fact that the non-local point-group symmetries in the energy spectrum become local spectral symmetries in the entanglement spectrum, as we have shown. The existence of these symmetry-protected gapless boundary states in the entanglement spectrum is a signature of a topological character, for it is dependent on the choice of boundary conditions. Whereas counting them relies explicitly on the point-group symmetries, as in Eq. (7.47) say, [13, 12] our main results (2.45) and (2.52) offer a complementary understanding to their stability.

\section{Acknowledgments}

We thank C. Chamon, C.-K. Chiu, M. J. Gilbert, C.-Y. Hou, C.-T. Hsieh, T. Morimoto, T. H. Taylor, and J. C. Y. Teo for insightful discussions.

\section{References}

[1] Hasan M Z and Kane C L 2010 Rev. Mod. Phys. 82(4) 3045-3067

[2] Qi X L and Zhang S C 2011 Rev. Mod. Phys. 83(4) 1057-1110

[3] Ryu S and Hatsugai Y 2006 Phys. Rev. B 73(24) 245115

[4] Levin M and Wen X G 2006 Phys. Rev. Lett. 96(11) 110405

[5] Kitaev A and Preskill J 2006 Phys. Rev. Lett. 96(11) 110404

[6] Li H and Haldane F D M 2008 Phys. Rev. Lett. 101(1) 010504

[7] Rodríguez I D and Sierra G 2009 Phys. Rev. B 80(15) 153303

[8] Fidkowski L 2010 Phys. Rev. Lett. 104(13) 130502

[9] Chen X, Gu Z C and Wen X G 2010 Phys. Rev. B 82(15) 155138

[10] Gu Z C, Wang Z and Wen X G 2010 arXiv: $1010.1517 v 1$

[11] Legner M and Neupert T 2013 Phys. Rev. B 88(11) 115114

[12] Turner A M, Zhang Y and Vishwanath A 2010 Phys. Rev. B 82(24) 241102

[13] Hughes T L, Prodan E and Bernevig B A 2011 Phys. Rev. B 83(24) 245132

[14] Yao H and Ryu S 2013 Phys. Rev. B 88(6) 064507

[15] Chiu C K, Yao H and Ryu S 2013 Phys. Rev. B 88(7) 075142 
[16] Morimoto T and Furusaki A 2013 Phys. Rev. B 88(12) 125129

[17] Shiozaki K and Sato M 2014 arXiv:1403.3331

[18] Hsieh T H, Lin H, Liu J, Duan W, Bansil A and Fu L 2012 Nat. Commun. 3982

[19] Xu S Y, Liu C, Alidoust N, Neupane M, Qian D, Belopolski I, Denlinger J D, Wang Y J, Lin H, Wray L A, Landolt G, Slomski B, Dil J H, Marcinkova A, Morosan E, Gibson Q, Sankar R, Chou F C, Cava R J, Bansil A and Hasan M Z 2012 Nat. Commun. 31192

[20] Tanaka Y, Ren Z, Sato T, Nakayama K, Souma S, Takahashi T, Segawa K and Ando Y 2012 Nat. Phys. 8 800-803

[21] Dziawa P, Kowalski B J, Dybko K, Buczko R, Szczerbakow A, Szot M, Łusakowska E, Balasubramanian T, Wojek B M, Berntsen M H, Tjernberg O and Story T 2012 Nat. Mater. 11 1023-1027

[22] Fu L 2011 Phys. Rev. Lett. 106(10) 106802

[23] Fang C, Gilbert M J, Dai X and Bernevig B A 2012 Phys. Rev. Lett. 108(26) 266802

[24] Fang C, Gilbert M J and Bernevig B A 2012 Phys. Rev. B 86(11) 115112

[25] Fang C, Gilbert M J and Bernevig B A 2013 Phys. Rev. B 87(3) 035119

[26] Jadaun P, Xiao D, Niu Q and Banerjee S K 2013 Phys. Rev. B 88(8) 085110

[27] Turner A M, Zhang Y, Mong R S K and Vishwanath A 2012 Phys. Rev. B 85(16) 165120

[28] Hou C Y, Chamon C and Mudry C 2007 Phys. Rev. Lett. 98(18) 186809

[29] Peschel I 2003 J. Phys. A: Math. Gen. 36 L205

[30] Witten E 1982 Nucl. Phys. B 202253 - 316 ISSN 0550-3213

[31] Streater R and Wightman A 1978 PCT, spin and statistics, and all that The mathematical physics monograph series (Benjamin/Cummings Publ. Co) ISBN 9780805392524

[32] Wu C S, Ambler E, Hayward R W, Hoppes D D and Hudson R P 1957 Phys. Rev. 105(4) 1413-1415

[33] Garwin R L, Lederman L M and Weinrich M 1957 Phys. Rev. 105(4) 1415-1417

[34] Christenson J H, Cronin J W, Fitch V L and Turlay R 1964 Phys. Rev. Lett. 13(4) 138-140

[35] Lees J P et al 2012 Phys. Rev. Lett. 109(21) 211801

[36] Schnyder A P, Ryu S, Furusaki A and Ludwig A W W 2008 Phys. Rev. B 78(19) 195125

[37] Schnyder A P, Ryu S, Furusaki A and Ludwig A W W 2009 AIP Conf. Proc. 113410

[38] Kitaev A 2009 AIP Conf. Proc. 113422

[39] Ryu S, Schnyder A P, Furusaki A and Ludwig A W W 2010 New J. Phys. 12065010

[40] Stone M, Chiu C K and Roy A 2011 J. Phys. A Math. Theor. 44045001

[41] Ryu S, Moore J E and Ludwig A W W 2012 Phys. Rev. B 85(4) 045104

[42] Novoselov K S, Geim A K, Morozov S V, Jiang D, Zhang Y, Dubonos S V, Grigorieva I V and Firsov A A 2004 Science 306(22) 666-669

[43] Novoselov K S, Geim A K, Morozov S V, Jiang D, Katsnelson M I, Grigorieva I V, Dubonos S V and Firsov A A 2005 Nature 438 197-200

[44] Castro E V, Grushin A G, Valenzuela B, Vozmediano M A H, Cortijo A and de Juan F 2011 Phys. Rev. Lett. 107(10) 106402

[45] Nomura K, Ryu S and Lee D H 2009 Phys. Rev. Lett. 103(21) 216801

[46] Hou C Y, Chamon C and Mudry C 2010 Phys. Rev. B 81(7) 075427

[47] Ghaemi P, Ryu S and Lee D H 2010 Phys. Rev. B 81(8) 081403

[48] Gomes K K, Mar W, Ko W, Guinea F and Manoharan H C 2012 Nature 483 306-310

[49] Ryu S, Mudry C, Hou C Y and Chamon C 2009 Phys. Rev. B 80(20) 205319

[50] Chamon C, Hou C Y, Jackiw R, Mudry C, Pi S Y and Schnyder A P 2008 Phys. Rev. Lett. 100(11) 110405

[51] Fujita M, Wakabayashi K, Nakada K and Kusakabe K 1996 J. Phys. Soc. Jpn. 65 1920-1923

[52] Ryu S and Hatsugai Y 2002 Phys. Rev. Lett. 89(7) 077002

[53] Yao W, Yang S A and Niu Q 2009 Phys. Rev. Lett. 102(9) 096801 